\title{
Frequently asked questions about chlorophyll fluorescence, the sequel
}

\author{
Hazem M. Kalaji ${ }^{1}$ - Gert Schansker ${ }^{2}$ - Marian Brestic ${ }^{3}$ - Filippo Bussotti ${ }^{4}$ • \\ Angeles Calatayud $^{5} \cdot$ Lorenzo Ferroni $^{6} \cdot$ Vasilij Goltsev $^{7} \cdot$ Lucia Guidi $^{8}$. \\ Anjana Jajoo ${ }^{9} \cdot$ Pengmin $\mathrm{Li}^{10}$ • Pasquale Losciale ${ }^{11}$ - Vinod K. Mishra ${ }^{12}$. \\ Amarendra N. Misra ${ }^{13} \cdot$ Sergio G. Nebauer ${ }^{14} \cdot$ Simonetta Pancaldi $^{6}$. \\ Consuelo Penella ${ }^{5}$ Martina Pollastrini ${ }^{4}$ - Kancherla Suresh ${ }^{15} \cdot$ Eduardo Tambussi $^{16}$. \\ Marcos Yanniccari ${ }^{16} \cdot$ Marek Zivcak $^{3} \cdot$ Magdalena D. Cetner $^{1} \cdot$ Izabela A. Samborska $^{1}$ • \\ Alexandrina Stirbet $^{17}$ - Katarina Olsovska ${ }^{18} \cdot$ Kristyna Kunderlikova $^{18}$. \\ Henry Shelonzek ${ }^{19} \cdot$ Szymon Rusinowski $^{20} \cdot$ Wojciech Bąba ${ }^{21}$
}

Received: 26 June 2016/ Accepted: 17 October 2016/Published online: 4 November 2016

(C) The Author(s) 2016. This article is published with open access at Springerlink.com

\begin{abstract}
Using chlorophyll (Chl) a fluorescence many aspects of the photosynthetic apparatus can be studied, both in vitro and, noninvasively, in vivo. Complementary techniques can help to interpret changes in the Chl $a$ fluorescence kinetics. Kalaji et al. (Photosynth Res 122:121-158, 2014a) addressed several questions about instruments, methods and applications based on Chl $a$ fluorescence. Here, additional Chl
\end{abstract}

Hazem M. Kalaji and Gert Schansker have contributed equally to this paper.

Hazem M. Kalaji

hazem@kalaji.pl

Gert Schansker

gert.schansker@gmail.com

Marian Brestic

marian.brestic@uniag.sk

Filippo Bussotti

filippo.bussotti@unifi.it

Angeles Calatayud

calatayud_ang@gva.es

Lorenzo Ferroni

lorenzo.ferroni@unife.it

Vasilij Goltsev

goltsev@gmail.com; goltsev@biofac.uni-sofia.bg

Lucia Guidi

lucia.guidi@unipi.it

Anjana Jajoo

anjanajajoo@hotmail.com

Pengmin Li

Lipm@nwsuaf.edu.cn

Pasquale Losciale

pasquale.losciale@crea.gov.it $a$ fluorescence-related topics are discussed again in a question and answer format. Examples are the effect of connectivity on photochemical quenching, the correction of $F_{V} / F_{M}$ values for PSI fluorescence, the energy partitioning concept, the interpretation of the complementary area, probing the donor side of PSII, the assignment of bands of $77 \mathrm{~K}$ fluorescence emission spectra to fluorescence emitters, the relationship between prompt and delayed fluorescence, potential problems when sampling tree canopies, the use of fluorescence parameters in QTL studies, the use of $\mathrm{Chl} a$ fluorescence in biosensor

Vinod K. Mishra

mishravkbhu@gmail.com

Amarendra N. Misra

misraan@yahoo.co.uk; misra.amarendra@gmail.com

Sergio G. Nebauer

sergonne@bvg.upv.es

Simonetta Pancaldi

simonetta.pancaldi@unife.it

Consuelo Penella

penella_con@gva.es

Martina Pollastrini

martina.pollastrini@unifi.it

Kancherla Suresh

sureshkancherla@rediffmail.com

Eduardo Tambussi

tambussi35@yahoo.es

Marcos Yanniccari

marcosyanniccari@conicet.gov.ar

Marek Zivcak

marek.zivcak@uniag.sk

Alexandrina Stirbet

stirbet@verizon.net 
applications and the application of neural network approaches for the analysis of fluorescence measurements. The answers draw on knowledge from different $\mathrm{Chl} a$ fluorescence analysis domains, yielding in several cases new insights.

Keywords Chl $a$ fluorescence · Delayed fluorescence . Photochemical quenching · Energy partitioning · Area

\section{Abbreviations \\ ANN}

Area, Sm

ATP

Car

Chl

${ }^{1} \mathrm{Chl},{ }^{3} \mathrm{Chl}$

$\mathrm{Chl}_{\mathrm{D} 1}$

CP43, CP47

CSm

Artificial neural network

Complementary area above the

fluorescence rise and this area normalized to $F_{V}$, respectively

Adenosine triphosphate

Carotenoid

Chlorophyll

Singlet chlorophyll and triplet chlorophyll

Accessory Chl molecule bound to the D1 protein

Core antenna proteins of PSII of 43 and $47 \mathrm{kDa}$, respectively Cross section (in the JIP test it is assumed that $F_{M}$ is a measure for the cross section)

Katarina Olsovska

katarina.olsovska@uniag.sk

Kristyna Kunderlikova

xkunderlikov@is.uniag.sk

Henry Shelonzek

shelonzek@gmail.com

Szymon Rusinowski

rusinowski@ietu.katowice.pl

Wojciech Bąba

wojciech.baba12@gmail.com

1 Department of Plant Physiology, Faculty of Agriculture and Biology, Warsaw University of Life Sciences - SGGW, Nowoursynowska 159, 02-776 Warsaw, Poland

2 Wesemlinstrasse 58, 6006 Lucerne, Switzerland

3 Department of Plant Physiology, Slovak Agricultural University, Tr. A. Hlinku 2, 94976 Nitra, Slovak Republic

4 Department of Agricultural, Food and Environmental Sciences, University of Florence, Piazzale delle Cascine 28, 50144 Florence, Italy

5 Departamento de Horticultura, Instituto Valenciano de Investigaciones Agrarias, Ctra. Moncada-Náquera Km 4.5., 46113 Moncada, Valencia, Spain

6 Department of Life Sciences and Biotechnology, University of Ferrara, Corso Ercole I d'Este, 32, 44121 Ferrara, Italy

7 Department of Biophysics and Radiobiology, Faculty of Biology, St. Kliment Ohridski University of Sofia, 8 Dr.Tzankov Blvd., 1164 Sofia, Bulgaria cyt

D1 protein

DCMU

ETC

ETR

Fd

FNR

$F_{O}, F_{M}, F_{O}{ }^{\prime}, F_{M}^{\prime}$

$F_{\text {PSI }}$

$F_{\mathrm{S}}$

$F_{V} / F_{M}$

IRGA

JIP test

K step
Cytochrome

One of the major PSII reaction center proteins, the other being D2

3-(3,4-Dichlorophenyl)-1,1-

dimethylurea

Electron transport chain

Electron transport rate

Ferredoxin

Ferredoxin $\mathrm{NADP}^{+}$reductase

Minimum and maximum

fluorescence intensity emitted by

dark- and light-acclimated samples, respectively

Chlorophyll $a$ fluorescence emitted by photosystem I

Steady-state chlorophyll

$a$ fluorescence

Maximum quantum yield of primary photosystem II photochemistry

Infrared gas analyzer

Analysis framework for the interpretation of OJIP transients developed by Bruno and Reto Strasser

Fluorescence intensity at $300 \mu \mathrm{s}$

8 Department of Agriculture, Food and Environment, Via del Borghetto, 80, 56124 Pisa, Italy

9 School of Life Sciences, Devi Ahilya University, Indore, M.P. 452 001, India

10 State Key Laboratory of Crop Stress Biology for Arid Areas, College of Horticulture, Northwest A\&F University, Yangling 712100, Shaanxi, China

11 Consiglio per la ricerca in agricoltura e l'analisi dell'economia agraria [Research Unit for Agriculture in Dry Environments], 70125 Bari, Italy

12 Department of Biotechnology, Doon (P.G.) College of Agriculture Science, Dehradun, Uttarakhand 248001, India

13 Centre for Life Sciences, Central University of Jharkhand, Ratu-Lohardaga Road, Ranchi 835205, India

14 Departamento de Producción vegetal, Universitat Politècnica de València, Camino de Vera sn., 46022 Valencia, Spain

15 ICAR - Indian Institute of Oil Palm Research, Pedavegi, West Godavari Dt., Andhra Pradesh 534 450, India

16 Institute of Plant Physiology, INFIVE (Universidad Nacional de La Plata - Consejo Nacional de Investigaciones Científicas y Técnicas), Diagonal 113 №495, CC 327, La Plata, Argentina

17204 Anne Burras Lane, Newport News, VA 23606, USA

18 Department of Plant Physiology, Slovak University of Agriculture, A. Hlinku 2, 94976 Nitra, Slovak Republic 
$\mathrm{kF}, \mathrm{kN}$ and $\mathrm{kP}$

Rate constants for $\mathrm{Chl}$

$a$ fluorescence, heat dissipation and photochemistry

LED

LHC, LHCI and

LHCII

Light-emitting diode

Light-harvesting complex, in general, associated with PSI and mainly associated with PSII, respectively

$\mathrm{M}_{o}$

The initial slope (first $250 \mu \mathrm{s}$ ) of the OJIP transient times 4, normalized to $F_{V}$

$\mathrm{NADP}^{+}$

NO

$\mathrm{NPQ}, q_{N}$

OEC

OJIP

P680, P700

PAR

PCA

$\mathrm{PF}, \mathrm{DF}$

Pheo

$P \mathrm{I}_{\mathrm{abs}}, P \mathrm{I}_{\mathrm{tot}}$

$P_{\mathrm{n}}, I_{\mathrm{PL}}$

PPFD

PsbO, PsbP and

PsbQ

PSI, PSII

$Q$ cycle

$Q_{A}, Q_{B}, \mathrm{PQ}$
Nicotinamide adenine dinucleotide

phosphate, oxidized form

Nitric oxide

Non-photochemical quenching expressed as $\left(F_{M} / F_{M}{ }^{\prime}-1\right)$ and $\left(1-F_{V}^{\prime} / F_{V}\right)$, respectively

Oxygen-evolving complex

Fluorescence rise on a dark-to-light transition from a minimum value $O$ via the intermediate steps $J$ and

$I$ to the maximum value $P$, which is

$F_{M}$ if the light is saturating

PSII and PSI reaction center chlorophyll dimer, respectively

Photosynthetically active radiance

Principal component analysis

Prompt fluorescence and delayed

fluorescence, respectively

Pheophytin, cofactor bound to PSII

Performance indexes of the JIP test

Net rate of carbon fixation and model based calculated net rate of carbon

fixation, respectively

Photosynthetic photon flux density

PSII extrinsic proteins

Photosystems I and II, respectively

Cyclic electron transport through cyt b6f and the PQ pool

Primary and secondary quinone electron acceptors of PSII and free plastoquinone, respectively
$q_{\mathrm{E}}$

$q_{\mathrm{P}}, q_{\mathrm{L}}$

QTL

$q_{Z}$

$\mathrm{RC}$

$R_{\mathrm{Fd}}$

RLC

ROS

Rubisco

RWC

SOM

SPAD

$S$ states

$S 0, S 1, S 2, S 3$ and

S4

$t_{F_{m}}$

$\mathrm{Tl}$

TL

TyrD, TyrZ

UV

$\mathrm{V}, \mathrm{A}, \mathrm{Z}$

VDE

$V_{J}, V_{I}$

$\Phi_{\mathrm{P} 0}$

$\Phi_{\mathrm{PSI}}, \Phi_{\mathrm{PSII}}$

$\psi E_{o}$

\section{Introduction}

In 2014 we published a paper in question and answer format on a series of chlorophyll (Chl) $a$ fluorescence-related topics (Kalaji et al. 2014a). There were, however, still enough questions left for a sequel. In the present paper we treat questions on the relationship between prompt fluorescence (PF), measured with fluorimeters like the PAM and the HandyPEA, and delayed fluorescence (DF), the
Energy quenching, fluorescence quenching dependent on an acidification of the lumen

Photochemical quenching calculated based on the puddle and lake model, respectively

Quantitative trait locus

Non-photochemical quenching of

Chl $a$ fluorescence related to the xanthophyll cycle

Reaction center

Relative fluorescence decrease ratio

Rapid light curve

Reactive oxygen species

Ribulose-1,5-bisphosphate

carboxylase/oxygenase

Relative water content

Self-organizing map

Refers to an instrument used to

estimate the leaf Chl content

Redox states of the oxygen-evolving complex

Time needed to rise from $O$ to $P$

Leaf temperature

Thermoluminescence

Tyrosine $\mathrm{D}$ and $\mathrm{Z}$, redox active tyrosines in the $\mathrm{D} 2$ and $\mathrm{D} 1$ proteins of PSII, respectively

Ultraviolet

Violaxanthin, antheraxanthin and zeaxanthin, respectively

Violaxanthin de-epoxidase

Relative position of the $J$ and $I$ steps between $O$ and $P$

Maximum quantum yield of primary photochemistry

PSI and PSII operating efficiency, respectively

JIP test parameter thought to be related to forward electron transport, defined as $1-V_{J}$

19 Department of Plant Anatomy and Cytology, Faculty of Biology and Environmental Protection, University of Silesia, ul. Jagiellońska 28, 40-032 Katowice, Poland

20 Institute for Ecology of Industrial Areas, Kossutha 6, 40-844 Katowice, Poland

21 Department of Plant Ecology, Institute of Botany, Jagiellonian University, Lubicz 46, 31-512 Kraków, Poland 
much weaker cousin of PF that is emitted in response to recombination reactions within PSII; energy partitioning; $q_{\mathrm{P}}$ versus $q_{\mathrm{L}}$; the analysis of several forms of stress using Chl $a$ fluorescence; the JIP test parameters area and $F_{J}$; the consequences of fluorescence emitted by PSI for parameters like $F_{V} / F_{M}$; considerations when sampling trees; the assignment of $77 \mathrm{~K}$ fluorescence bands; QTL studies on $\mathrm{Chl} a$ fluorescence-related traits from a Chl $a$ fluorescence point of view and several other topics.

\section{Question 1: What is chlorophyll $a$ fluorescence and why do we study it?}

Chl $a$ fluorescence can be defined as the red to far-red light emitted by photosynthetic tissues/organisms when illuminated by light of approximately 400-700 nm (photosynthetically active radiation or PAR) (McCree 1972). Within this spectrum, blue and red light excite chlorophyll more efficiently than green light. Although Chl $a$ fluorescence represents only a small fraction of the absorbed energy [approximately 0.5-10\% (Latimer et al. 1956; Brody and Rabinowitch 1957; Barber et al. 1989; Porcar-Castell et al. 2014)], its intensity is inversely proportional to the fraction of energy used for photosynthesis (a redox effect) (Duysens and Sweers 1963). For this reason, the Chl $a$ fluorescence signal can be used as a probe for photosynthetic activity. At the same time, Chl $a$ fluorescence is also inversely proportional to changes in dissipative heat emission (a yield effect, i.e., an increase in the yield of heat emission causes a decrease in the yield of fluorescence emission) (e.g., Krause and Weis 1991) and, therefore, Chl $a$ fluorescence can be used as well to monitor regulatory processes affecting the PSII antenna (see, e.g., Question 8). Finally, $\mathrm{P}_{680^{+}}$is a strong quencher of Chl $a$ fluorescence (Steffen et al. 2005) and this effect allows the study of the different redox states (S states) the oxygen-evolving complex of PSII, due to the fact that the lifetime of $\mathrm{P} 680^{+}$is $S$ state dependent. All of these things taken together could turn Chl $a$ fluorescence into a indecipherable signal, but thanks to the development of specific protocols, and by using complementary techniques, the different effects can be separated, turning Chl $a$ fluorescence into a powerful tool for the study of photosynthesis: quenching analysis (Bradbury and Baker 1981; Quick and Horton 1984; Schreiber et al. 1986), JIP test (Strasser and Strasser 1995; Strasser et al. 2004), non-photochemical quenching (NPQ) (Demmig and Winter 1988; Horton and Hague 1988), electron transport rate (ETR) (Genty et al. 1989; Krall and Edwards 1990), rapid light curves (RLCs) (White and Critchley 1999; Ralph and Gademann 2005), flash-induced fluorescence (Robinson and Crofts 1983; de Wijn and van Gorkom 2001; Bouges-Bocquet 1980, Ioannidis et al.
2000), dark-adaptation kinetics of OJIP transients (Bukhov et al. 2001; Schansker et al. 2005), Chl $a$ fluorescence and photoacoustic spectroscopy (Buschmann and Koscányi 1989; Snel et al. 1990; Allakhverdiev et al. 1994; Bukhov et al. 1997), Chl $a$ fluorescence and 820-nm absorbance/transmission (Klughammer and Schreiber 1994; Schansker et al. 2003), Chl $a$ fluorescence and delayed fluorescence (Goltsev et al. 2012; Kalaji et al. 2012a), imaging (Nedbal and Whitmarsh 2004; Hideg and Schreiber 2007; Lichtenthaler et al. 2007; Gorbe and Calatayud 2012), the actinic light wavelength dependence of photosynthesis (Schreiber et al. 2012) and more recently attention has been paid to statistic aspects of the measurements of parameters (e.g., Bussotti et al. 2011a). The photosynthetic literature is huge with many topics studied such as plant breeding (Baker and Rosenqvist 2004; Kalaji and Pietkiewicz 2004; Kalaji and Guo 2008), seed vigor and seed quality assessment (Jalink et al. 1998; Dell'Aquila et al. 2002; Konstantinova et al. 2002), fruit and vegetable quality determination and postharvest processing control (Merz et al. 1996; Nedbal et al. 2000), senescence (Adams et al. 1990a; Kotakis et al. 2014), climate change effects (Ashraf and Harris 2004) and a variety of algae (Gorbunov et al. 1999; Antal et al. 2009; Grouneva et al. 2009). Furthermore, Chl $a$ fluorescence measurements have been used for monitoring plant stresses (Guidi and Calatayud 2014), such as photoinhibition (Sarvikas et al. 2010; Matsubara et al. 2011), heat stress (Allakhverdiev et al. 2007; Ducruet et al. 2007; Tóth et al. 2007a; Kalaji et al. 2011a; Brestič et al. 2012), UV stress (Vass et al. 1999; van Rensen et al. 2007; Guidi et al. 2011), salt stress (Kalaji and Pietkiewicz 1993; Demetriou et al. 2007; Melgar et al. 2009; Kalaji et al. 2011b; Penella et al. 2016), drought stress (Lu and Zhang 1998; Flexas et al. 2002; Živčák et al. 2013), urban tree conditions (Hermans et al. 2003; Swoczyna et al. 2010a, b), environmental pollution (Bussotti et al. 2005; Kalaji and Łoboda 2007; Romanowska-Duda et al. 2010; Tuba et al. 2010; Bussotti et al. 2011b; Cotrozzi et al. 2016), sulfur-deprivation/ $\mathrm{H}_{2}$ production in Chlamydomonas (Antal et al. 2007; Nagy et al. 2012) and water quality (Romanowska-Duda et al. 2005; Ralph et al. 2007; Baumann et al. 2009).

\section{Question 2: Does Chl $a$ fluorescence only probe PSII?}

A common misunderstanding is that variable Chl $a$ fluorescence is a specific probe for PSII. This is true for flash experiments, in which $Q_{A}$ in all PSII RCs is reduced by a saturating single turnover flash. However, if longer pulses of light are given, $Q_{A}$ will become reduced and oxidized multiple times, and under these conditions fluorescence 
also becomes a probe for the reduction and redox state of the PQ pool and even for the electron flow through PSI and PSI content (Schansker et al. 2005; Ceppi et al. 2012).

Under steady-state conditions, i.e., a stable level of photosynthesis reached after a few minutes of illumination, the whole photosynthetic apparatus is in equilibrium and electron flow through any of the components of the electron transport chain (including PSII) would be indicative for the overall photosynthetic rate (Kramer et al. 2004a; Scheibe et al. 2005; Eichelmann et al. 2009). As a consequence, under steady-state conditions, the electron flux calculated on the basis of the Chl $a$ fluorescence signal can be used as a measure for the overall photosynthetic activity. This point was demonstrated by Genty et al. (1989, 1990a).

Another common mistake is to interpret fluorescence measurements in terms of single reaction centers. In the case of photoinhibition it is, e.g., often assumed or implied that the quantum yield of individual PSII RCs changes, whereas it is more realistic to interpret changes in the parameter $F_{V} / F_{M}$ in terms of changes in the quantum yield of the population of PSII RCs as a whole.

The importance of looking at photosynthesis measurements in stochastic terms can be illustrated by experiments showing that at high light intensities $80 \%$ of the PSII RCs can be inhibited before the electron transport rate becomes affected (e.g., Heber et al. 1988).

This observation also illustrates that at high light intensities PSII activity has little relevance for photosynthetic activity, whereas at low light intensities PSII RCs become rate limiting. This also means that the effect of a treatment on PSII measured at a single light intensity has limited meaning.

\section{Question 3: What is the Kautsky effect?}

Kautsky and Hirsch (1931) observed for several types of leaves that a dark-to-light transition is characterized by an initial fast increase of the fluorescence intensity followed by a slow decrease to a minimum level, after which the fluorescence intensity remains at this low intensity. The authors assigned the stable low level of fluorescence to steady-state photosynthesis. They noted further that the slow fluorescence decrease had the same time dependence as the induction of $\mathrm{CO}_{2}$ assimilation and concluded that the fast fluorescence rise reflects a photochemical reaction since it was insensitive to cyanide and temperature changes. The fluorescence changes occurring during induction of photosynthesis have been studied intensively during the last 50 years and, in honor of the first publication on this phenomenon, such a fluorescence transient is called a Kautsky transient, and the changes in the fluorescence intensity the Kautsky effect. In Fig. 1 examples of the first $10 \mathrm{~s}$ of Kautsky transients measured on several angiosperm and gymnosperm plants are shown on a logarithmic timescale. The fluorescence rise phase (OJIP) reflects the reduction of the photosynthetic electron transport chain (see Kalaji et al. 2014a for a more comprehensive discussion) and its kinetics, as illustrated in Fig. 1, are quite similar for all photosynthetic organisms. The fluorescence decrease has kinetics that differ quite strongly between different types of photosynthetic organisms (in Fig. 1 angiosperm vs. gymnosperm plants). The $S$ and $M$ steps observed in transients of gymnosperm species lack/are hidden in transients of angiosperm species. Using 820-nm transmission measurements it was shown that the initial fluorescence kinetics beyond $P$ depend strongly on the activation of electron flow at the PSI acceptor side, associated with the activation of ferredoxin-NADP ${ }^{+}$reductase (FNR) (Kautsky et al. 1960; Munday and Govindjee 1969; Satoh 1981; Harbinson and Hedley 1993; Schansker et al. 2003, 2008; Ilík et al. 2006). Fluorescence then declines within 3-5 min with the onset of photosynthetic $\mathrm{CO}_{2}$ fixation until it reaches a lower, steady-state fluorescence intensity $\left(F_{\mathrm{S}}\right)$. In fully photosynthetically active leaves this steady-state level, especially at high light intensities, is usually close to the $F_{O}$ level (e.g., Flexas et al. 2002).

\section{Question 4: What is quantum yield?}

In a general sense, the quantum yield can be defined by an action, e.g., oxygen evolution or a stable charge separation, divided by the number of photons that has to be absorbed

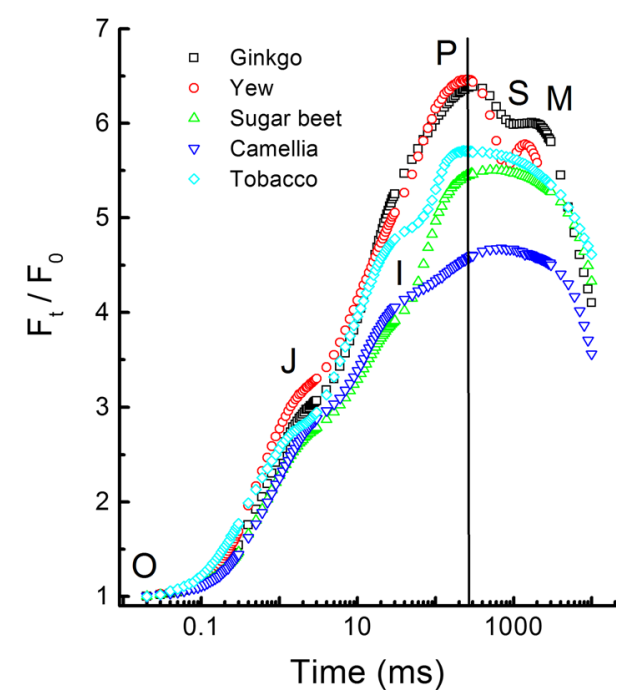

Fig. $1 \mathrm{Chl} a$ fluorescence induction transients measured on angiosperm (sugar beet, camellia and tobacco) and gymnosperm (Ginkgo and yew) leaves. The fast induction kinetics OJIP are similar for both types of plants with a higher $F_{M} / F_{O}$ ratio in gymnosperms and the same OJIP kinetics for all leaves/needles measured. Beyond $P$ the kinetics differ quite strongly between both types of plants (Schansker et al., unpublished data) 
for this action. The quantum yield of oxygen evolution has been studied intensively (Warburg and Negelein 1923; Emerson and Lewis 1943; Govindjee 1999). Govindjee et al. (1968) concluded for Chlorella cells that the quantum yield for oxygen evolution is at least 0.12 , which means that at least 8 light quanta are needed for this process. The maximum quantum yield of a stable charge separation for the dark-adapted state is in the literature defined as $F_{V} / F_{M}$, and this gives a value of about 0.88 in higher plants (see Question 6). Tyystjärvi and Aro (1996) determined a quantum yield for the photoinhibition of PSII of $7 \times 10^{-8}$, which means that for every 14.3 million photons absorbed, one PSII RC is inactivated. For each photochemical process such a quantum yield can be determined.

If we look at the potential fate of a single photon that has excited a chlorophyll molecule, the sum of the different deexcitation pathways, due to the law of energy conservation, is 1. The three main de-excitation pathways are photochemistry (induction of a stable charge separation), emission as heat, and emission as Chl $a$ fluorescence. In open PSII RCs photochemistry is the fastest process and has the highest probability/quantum yield (see also Questions 6 and 13). In more physical terms the quantum yield of photochemistry is the rate constant for photochemistry divided by the sum of the rate constants of all competing processes (photochemistry, heat dissipation, Chl $a$ fluorescence emission) [for a more in-depth treatment of this topic: Harbinson and Rosenqvist (2003), Strasser et al. (2004) and Lazár (2016)].

Since photochemistry, fluorescence and heat are competing de-excitation processes, fluorescence measurements can be used to assess the balance between photochemistry and non-photochemical dissipation of absorbed light quanta (photons) under different environmental conditions. It is important to keep in mind that more fluorescence means either less photochemistry and/or less heat (see also Question 13).

\section{Question 5: When are reaction centers considered to be closed?}

The biochemical definition of a closed reaction center is simple. If $Q_{A}$ is in the reduced state $\left(Q_{A}^{-}\right)$no further stable charge separations can occur and the rate constant for photochemistry $(\mathrm{kP})$ goes to 0 (the presence of $\mathrm{P} 680^{+}$ will also close PSII, but this we will not treat here; see for $\mathrm{P}_{680}{ }^{+}$Questions 18 and 22). Unfortunately, the redox state of $Q_{A}$ cannot be measured directly under most conditions; therefore, Chl $a$ fluorescence is used instead. Based on the paper of Duysens and Sweers (1963) a closed RC is generally equated to $F_{M}$. However, looking at the literature, the $F_{M}$ value depends on the technique used to determine it. A single turnover xenon or laser flash is thought to reduce $Q_{A}$ in all reaction centers. However, the $F_{M}$ measured under these conditions is 30-50\% lower than the $F_{M}$ induced by a saturating pulse of 200-500 ms (Samson and Bruce 1996). In addition, even at very high light intensities (12,000-15,000 $\mu \mathrm{mol}$ photons $\left.\mathrm{m}^{-2} \mathrm{~s}^{-1}\right)$, where the excitation rate is once every 40-50 $\mu$ s (Neubauer and Schreiber 1987; Lazár and Pospíśil 1999), which is considerably higher than the re-oxidation time of $Q_{A}^{-}$of $100-200 \mu \mathrm{s}$ in the presence of $Q_{B}$ and $400-600 \mu$ s in the presence of $Q_{\mathrm{B}}^{-}$ (Petrouleas and Crofts 2005), it still takes 80-100 ms to reach $F_{M}$ (Schreiber 1986; Neubauer and Schreiber 1987; Schansker et al. 2006). From a practical point of view, it can be argued that the $F_{M}$ represents a state with all RCs closed for both single turnover flashes and saturating pulses. The difference is that during a saturating pulse many other things happen as well that affect the fluorescence intensity and, therefore, the $F_{M}$ values of flash and pulse experiments are not directly comparable.

\section{Question 6: How can fluorescence measurements and derived fluorescence parameter be corrected for fluorescence emission by PSI?}

As noted in the previous paper (Kalaji et al. 2014a), at wavelengths longer than $700 \mathrm{~nm}$ PSI fluorescence emission contributes considerably to $F_{O}$. For commercial fluorimeters this contribution may be as high as $30-35 \%$ for $\mathrm{C} 3$ plants and 50-60\% for C4 plants (Genty et al. 1990b; Adams et al. 1990b; Pfündel 1998; Peterson et al. 2001). The stronger contribution of PSI fluorescence $\left(F_{\mathrm{PSI}}\right)$ in $\mathrm{C} 4$ plants is due to a higher PSI/PSII ratio (Edwards and Walker 1983; Ku et al. 1991) and to higher levels of spillover of excitation energy from PSII to PSI (Pfündel and Pfeffer 1997). The question whether PSI emits variable fluorescence at room temperature has been studied as well. It is often assumed that the fluorescence yield of open and closed RCs of PSI is the same (Butler 1978; Kyle et al. 1983; Savikhin 2006). Byrdin et al. (2000) reported a $12 \%$ increase of the fluorescence yield of PSI of Synechococcus elongatus on closing. If $F_{\mathrm{PSI}}$ is $30 \%$ of the $F_{O}$ fluorescence emission, then $12 \%$ more would be equal to $4 \%$ of $F_{O}$, and, since $F_{M}$ is 5-6 times $F_{O}$, this would represent $1 \%$ or less of the total variable fluorescence. In other words, even if there is some PSI variable fluorescence, this amount is so small that it can be ignored. This is further supported by several kinetic experiments. In leaves or intact chloroplasts, in the presence or absence of DCMU, the $F_{M}$ is the same (Schreiber and Krieger 1996; Tóth et al. 2005b) despite the fact that in the absence of DCMU P700 is reduced at $F_{M}$ and in its presence is oxidized (Schansker et al. 2005). In a variation on this experiment Peterson et al. 
(2014) showed that during fluorescence induction $\left(F_{O}\right.$ to $F_{M}$ ) the relationship between $\mathrm{F}(680)$ (more PSII fluorescence) and $\mathrm{F}(750)$ (more PSI fluorescence) did not show an oscillation related to the P700 oxidation and reduction kinetics occurring during OJIP fluorescence rise. Peterson et al. (2014) concluded that variable PSI fluorescence was less than $0.8 \%$ of $F_{V}$. In contrast, theoretical simulations performed by Lazár (2013), based on known values of rate constants of PSI reactions and considering the reported PSII/PSI stoichiometry, yielded an OJIP simulation with approximately correct kinetics. On the basis of these results Lazár concluded that the contribution of PSI variable $\mathrm{Chl}$ $a$ fluorescence to total $F_{V}$ could be $8-17 \%$. However, a close link between PSI kinetics and the OJIP rise can also be explained on the basis of the PSII conformational change hypothesis (Schansker et al. 2014).

Several authors have studied methods to correct parameters like $F_{V} / F_{M}$ for the contribution of PSI fluorescence, but, so far, this has not led to a simple formula that can be applied. It is important to note that the PSI contribution is instrument sensitive. Pfündel (1998) wrote that a special PAM instrument that detects the fluorescence emission at wavelengths shorter than $710 \mathrm{~nm}$ shows very little, or at least much less, contribution of PSI fluorescence.

Pfündel (1998) showed for a set of C3, C3-C4 and C4 plants that there is a linear relationship between the parameter $F_{M} / F_{V}$ determined at room temperature and the parameter F735/F685 determined at $77 \mathrm{~K}$, with a slope $m$ and an intercept of the $Y$ axis $b$. In the model of Pfündel (1998):

$F_{M} / F_{V}=b+m \times \mathrm{F} 735 / \mathrm{F} 685$

For the data set of Pfündel (1998) this gave a regression coefficient of 0.963 . On the basis of this approach, a corrected $F_{V} / F_{M}$ value of about 0.88 was obtained. To use this approach, it would be necessary to construct a calibration curve, like Pfündel (1998) did, for each instrument used and then to determine for the samples of interest both the $F_{O}$ and $F_{M}$ at room temperature measured on leaves and the $77 \mathrm{~K}$ fluorescence emission spectrum of diluted leaf powder, which in most cases is impractical.

Franck et al. (2002) approached the topic in a different way, developing a method for the resolution of the PSII and PSI contributions to the fluorescence emission spectrum. The authors noted that, for diluted PSII particles, the $F_{M} l$ $F_{O}$ was wavelength independent. On that basis, they concluded that the wavelength dependence of $F_{M} / F_{O}$ observed for leaves was due to the presence of PSI. Furthermore, they assumed that the PSI and PSII spectra do not change and, therefore, that these spectra can be scaled to obtain the $F_{O}$ and $F_{M}$ spectra. After correction by this method, the authors obtained a $F_{V} / F_{M}$ value of 0.83 instead of 0.81 .
This difference is considerably smaller than the correction found by Pfündel (1998).

The quantum yield of PSII can also be determined on the basis of time-resolved (ps) fluorescence measurements. Wientjes et al. (2013a) acclimated Arabidopsis plants to 20, 100 and $800 \mu \mathrm{mol}$ photons $\mathrm{m}^{-2} \mathrm{~s}^{-1}$. Under such conditions the PSII antenna size decreased as the light intensity increased. The quantum yields derived from the time-resolved fluorescence measurements were $0.84,0.89$ and 0.91 , respectively. The $F_{V} / F_{M}$ values (corrected for the PSI contribution) determined for the same plants were 0.83 , 0.87 and 0.86 , respectively. Since the first set of data is measured on thylakoid membranes and the second set of data on leaves, there are several possible explanations for the observed discrepancies.

The data of Wientjes et al. (2013a) support the choice of a $F_{V} / F_{M}$ value of 0.87 or 0.88 as a good approximation of the real $F_{V} / F_{M}$ value, at least for $\mathrm{C} 3$ plants. Taking 0.88 as the real value of the parameter $F_{V} / F_{M}$ of PSII $\left(=\Phi_{\mathrm{P} 0}\right)$ of C3 and $\mathrm{C} 4$ plants, it can be used to estimate the contribution of PSI fluorescence:

$F_{\mathrm{PSI}}=\left[\left(\phi_{P 0} \cdot \frac{F_{m}}{F_{m}-F_{o}}\right)-1\right] \cdot F_{m}$

when we take a typical $F_{V} / F_{M}$ value for C3 plants (e.g., 0.836 ), we get $F_{\mathrm{PSI}}=\sim 5.2 \%$ of $F_{M}$. When we take a typical value for $\mathrm{C} 4$ plants (e.g., 0.80), we get $F_{\mathrm{PSI}}=\sim 10 \%$ of $F_{M}$. This calculation can, however, only be applied to $F_{O}$ and $F_{M}$ measurements on plants that are completely relaxed with respect to photoprotective dissipation mechanisms (no NPQ) and non-stressed (no photoinhibition). The data of Wientjes et al. (2013a) also suggest that 0.88 is too high for plants acclimated to shade conditions. Another approach will also have to be developed for the correction of the $F_{V} / F_{M}$ values in the photosynthetic organisms in which the thylakoid stacking is hindered by the presence of phycobilisomes (cyanobacteria, red algae), or the thylakoids are appressed for their entire length (brown algae, diatoms, etc.), or display a not yet well-differentiated grana-intergrana arrangement (most green algae) (see Trissl and Wilhelm 1993; Solymosi 2012). Further, Peterson et al. (2014) described an additional contribution to $F_{O}$ in greening maize (up to $12-15 \%$ of $F_{M}$ at $680 \mathrm{~nm}$ ) and sunflower (up to $8 \%$ of $F_{M}$ at $680 \mathrm{~nm}$ ) leaves which was absent in mature leaves and correcting for which improved the analysis of the fluorescence data. The authors ascribed this fluorescence to emission by partially assembled PSII and could be the same fluorescence emission Srivastava et al. (1999) ascribed to free LHCII. Once $F_{\mathrm{PSI}}$ has been determined, it can be subtracted from all $F_{t}$ values and the resulting fluorescence data can be used for the calculation of all fluorescence parameters. 
A correction of fluorescence measurements for the PSI contribution is especially relevant when fluorescence measurements are correlated with data obtained by other methods (e.g., gas exchange or absorbance measurements).

Strong red LEDs with a peak emission at $\sim 650 \mathrm{~nm}$ were the first LEDs that became commercially available for a reasonable price. Instruments that use such LEDs need to measure fluorescence above $700 \mathrm{~nm}$ to avoid overlap with the emission of the red LEDs. This is the case for, e.g., classical PAM instruments and HandyPEAs. Using, e.g., blue LEDs it is possible to avoid the overlap problem and to measure fluorescence emission at $\sim 685 \mathrm{~nm}$, where the contribution of PSI fluorescence is very small (Krause and Weis 1991; Gitelson et al. 1998). However, Peterson et al. (2001, 2014) argued that in the end the fluorescence detected above $700 \mathrm{~nm}$ may be the better probe, because light around $680 \mathrm{~nm}$ is much more strongly absorbed by the leaf and, therefore, more a probe for the top cell layers of the leaf. Further, it should be noted that differences in filters and other specifications between instruments may affect the contribution of PSI to fluorescence measurements and can explain, at least to some extent, differences in the values of parameters like $F_{V} / F_{M}$ between different fluorometers.

Part of the JIP test parameters (e.g., $\mathrm{M}_{o}$, Area, Sm, $V_{J}$, $V_{I}, \psi E_{o}$ ) only depend on variable fluorescence and are not affected by the contribution of PSI fluorescence. For measurements derived from OJIP measurements it may be noted that, as long as the PSII to PSI ratio does not change, PSI fluorescence causes a systematic error. This means that it does not affect the comparability of measurements of comparable samples. With respect to the quenching analysis, the effect of PSI fluorescence emission on the calculated parameters increases for measurements made at stronger actinic light intensities. Higher light intensities quench $F_{M}$, and to a lesser extent $F_{O}$, but are not expected to affect $F_{\mathrm{PSI}}$, increasing the relative contribution of $F_{\mathrm{PSI}}$. Pfündel et al. (2013) studied the effects of $F_{\mathrm{PSI}}$ under steady-state conditions. They noted that the method of Oxborough and Baker (1997) to calculate $F_{O}{ }^{\prime}$ systematically produces values that are too low and they ascribed this to the fact that Oxborough and Baker (1997) did not take the contribution of $F_{\mathrm{PSI}}$ into account. Pfündel et al. (2013) also showed that correcting fluorescence data of maize for $F_{\mathrm{PSI}}$ makes the relationship between $\Phi_{\mathrm{PSI}}$ and $\Phi_{\mathrm{PSII}}$ more linear.

In summary, PSI fluorescence emission has only a significant effect on $F_{O}$. Even a rough correction of fluorescence data for PSI fluorescence emission, assuming that the real $F_{V} / F_{M}$ value is 0.88 , will considerably improve the quality of the fluorescence data.

\section{Question 7: How does cytochrome $b_{6} / f$ regulate and affect the redox state of the photosynthetic electron transport and parameters like $q_{\mathrm{E}}$ and ETR?}

The cytochrome (cyt) $b_{6} / f$ complex is located between PSII and PSI at a crossroad of different electron pathways (linear electron transport, $Q$ cycle, chlororespiration, cyclic electron transport) (Sacksteder et al. 2000; Bennoun 2002; Mulkidjanian 2010; Johnson 2011; Shikanai 2014) and is an important site for the regulation of electron flow and the control of regulatory mechanisms like state transitions (see Question 8 ) and $q_{\mathrm{E}}$. The $Q$ cycle and cyclic electron transport increase the ATP to NADPH ratio by diverting electrons away from $\mathrm{NADP}^{+}$while at the same time increasing the $\mathrm{pH}$ difference over the membrane (Sacksteder et al. 2000; Munekage et al. 2010; Johnson 2011). Lowering the lumen $\mathrm{pH}$ decreases the re-oxidation rate of $\mathrm{PQH}_{2}$, thereby slowing down electron transport (Witt 1979; Heber et al. 1988; Harbinson et al. 1990). A low lumen pH is also the driving force behind $q_{\mathrm{E}}$. Kramer et al. (1999), reviewing the literature on the lumen $\mathrm{pH}$, argued that under most conditions the lumen $\mathrm{pH}$ remains between $\mathrm{pH} 5.8$ and 6.3. The feedback inhibition of $\mathrm{PQH}_{2}$ oxidation may play an important role in keeping the lumen $\mathrm{pH}$ within this range. At the same time, this feedback inhibition will lead to a more reduced PQ pool where the cyt $b_{6} / f$ complex is known as a sensor for the PQ pool redox state, activating a kinase that can phosphorylate LHCII when the PQ pool becomes more reduced; this is the classical definition of state transitions (see Question 8).

Under steady-state conditions, a higher light intensity means a more reduced PQ pool and a more oxidized PSI donor side (Klughammer and Schreiber 1994; Živčák et al. 2014). This is due to the fact that PSII can pump electrons faster to the PQ pool and PSI can pump them faster to the electron acceptors at its acceptor side than cyt $b_{6} / f$ can transfer them from the PQ pool to plastocyanin and, then, P700. This imbalance increases as the light intensity is raised and can be detected by measuring Chl $a$ fluorescence and 820-nm transmission/absorption simultaneously (Klughammer and Schreiber 1994; Živčák et al. 2014). The described feedback mechanism can respond rapidly to fluctuations in the light intensity and will keep PSI in a relatively oxidized state. The excitation quenching ability of $\mathrm{P} 700^{+}$state of PSI has recently been suggested to play a photoprotective role, since in the cyanobacterium Arthrospira platensis $\mathrm{P} 700^{+}$photostability was shown to reduce PSI photodestruction (Shubin et al. 2008). A similar mechanism was hypothesized to be operative in vascular plants as well (Tikkanen and Aro 2014; Ferroni et al. 
2014). A similar observation (sensitivity of PSI to a reduced acceptor side) was made for plants in which cyclic electron transport was inactivated and the plants were exposed to fluctuating light conditions (Suorsa et al. 2012). Tikkanen et al. (2014) have argued that the ability of the chloroplast apparatus to keep PSI in a relatively oxidized state is critical, because damage to PSI is nearly irreversible. It is this aspect that makes the cyt $b_{6} / f$ complex a particularly important regulatory point for electron flow under conditions of changing light conditions (Genty and Harbinson 2004). Tikkanen et al. (2015) recently concluded that the cyt $b_{6} / f$ complex and not downregulation of PSII by the processes related to $q_{\mathrm{E}}$ regulates linear electron transport. They based this on the observation that PsbS-less npq4 mutant of Arabidopsis thaliana has an impaired ability to generate $q_{\mathrm{E}}$, but still combines a highly reduced PQ pool with strongly oxidized P700 in high light, indicating that the ability of cyt $b_{6} / f$ to control electron flow is retained. This supports the observation of Belgio et al. (2014) that processes related to $q_{\mathrm{E}}$ do not reduce the efficiency of PSII to trap excitation energy.

\section{Question 8: What is a state transition and how does it affect $\mathrm{Chl} a$ fluorescence?}

Bonaventura and Myers (1969) were the first to describe state transitions in cells of Chlorella only a few years after the existence of two photosystems had been shown (Duysens et al. 1961). Duysens et al. (1961) had used light of 560 and $680 \mathrm{~nm}$ to preferentially excite either of those two photosystems. This was done as well by Bonaventura and Meyers by using so-called light 2 of $645 \mathrm{~nm}$ exciting PSII and PSI and light 1 of $710 \mathrm{~nm}$ preferentially exciting PSI. The authors observed slow excitation-wavelength-dependent changes in the $\mathrm{O}_{2}$ evolution rate and Chl $a$ fluorescence, which they interpreted as a change in the distribution of light energy between the two photosystems. In 1977, John Bennett showed that in the light several photosynthetic proteins became phosphorylated (especially LHCII and a 9-kDa protein) and he suggested a link with the above-described state transitions (Bennett 1977). Subsequently, it was shown that a reduced PQ pool was needed to activate the kinase that phosporylated LHCII and that cyt $b_{6} / f$ acted as a redox sensor (Allen et al. 1981; Bennett et al. 1988; Rintamäki et al. 2000).

In the literature, several methods can be found to detect state transitions. A variation of the experimental approach of Bonaventura and Myers (1969) is the determination of the effect of pre-illumination with PSII and PSI light on $F_{M}{ }^{\prime}$ induced by a saturating pulse (e.g., Lunde et al. 2000). A variant of this approach is to use the $F_{S} / F_{M}{ }^{\prime}$ ratio induced in response to either PSI or PSII light (Wagner et al. 2008).
Emission spectra ( $77 \mathrm{~K}$ ) are also widely used to detect state transitions. In green algae such as Chlamydomonas reinhardtii, in which state 2 is induced by anaerobic conditions that cause a reduction of the PQ pool, this works very well (Depège et al. 2003; Iwai et al. 2008). On going from state 1 to state 2, the PSII bands decrease and the PSI bands increase in amplitude. A variation on this approach is to use the F735/F685 ratio (at $77 \mathrm{~K}$ ) as a measure for state transitions. This ratio increases during the transition from state 1 to state 2 and decreases during the transition from state 2 to state 1 as, e.g., demonstrated by McCormac et al. (1994) for Spirodela oligorrhiza. Studying OJIP transients, Schreiber et al. (1995) observed that state 2 in C. reinhardtii and Synechocystis PCC 6803 is associated with a reduction in the JI amplitude. Schansker et al. (2006) observed that already at low actinic light intensities the JI amplitude in the steady state decreased, an effect that had still not reversed after $15 \mathrm{~min}$ of dark adaptation. The authors interpreted this in analogy with Schreiber et al. (1995) as the effect of a state 1 to state 2 transition. Recently, a simple model to simulate state transitions in $C$. reinhardtii was created (Ebenhöh et al. 2014) based on which Stirbet and Govindjee (2016) tried to simulate the slow PS(M)T fluorescence decline.

Depège et al. 2003 identified the kinase (Stt7) in $C$. reinhardtii, and then, Bellafiore et al. (2005) identified its higher plant ortholog (STN7) in Arabidopsis thaliani. However, in the STN7 mutant, which lacked this gene had a phenotype that was very similar to that of the wild type. Only under fluctuating light conditions, the growth of the STN7less mutant was affected. Grieco et al. (2012) observed that in the STN7 mutant grown under fluctuating white light the PSI content decreases and they proposed, therefore, that state transitions are important in protecting PSI against damage under fluctuating white light conditions.

According to the classical concept of state transitions, the phosphorylated LHCII disconnects from PSII and migrates to PSI, which leads to a redistribution of excitation energy from PSII to PSI (Allen 1992; McCormac et al. 1994; Misra and Biswal 2000). This view has been modified in recent years. Wientjes et al. (2013b) showed that LHCII acts as an efficient antenna for both photosystems under most naturally occurring conditions and that only under special conditions (strong preferential excitation of PSI with FR light or strong light) LHCII migrates to PSII, where, especially under high light conditions, the light it absorbs can be more easily quenched. Grieco et al. (2015) looked at PSII and PSI as located in a connected lake of LHCIIs. For high light conditions, it has been shown that the LHCII kinase becomes inactivated by reduced thioredoxins leading to the dephosphorylated state of LHCII (Rintamäki et al. 2000).

In summary, since its discovery in 1969 the role of state transitions in higher plants has evolved and is still 
evolving. Based on recent studies on plants lacking the LHCII kinase, state transitions are thought to play a regulatory role under fluctuating light conditions, possibly protecting PSI. Its role in the redistribution of light energy, in higher plants, seems to be less important. For a comprehensive review of this topic, see, e.g., GoldschmidtClermont and Bassi (2015).

\section{Question 9: How can photochemical quenching be defined and what type of information does it provide?}

Photochemical quenching is a reflection of the redox state of $Q_{A}$. If the photosynthetic electron transport chain is oxidized, re-oxidation of $Q_{A}$ by forward electron transfer can compete strongly with fluorescence emission and can keep the fluorescence intensity low (Kautsky et al. 1960; Munday and Govindjee 1969; Bradbury and Baker 1981; Krause et al. 1982). This is called photochemical quenching (e.g., Bradbury and Baker 1981). If the relationship between $Q_{A}$ redox state and variable fluorescence were linear, as proposed by Duysens and Sweers (1963), we could simply use the parameter $q_{\mathrm{P}}$ as it was defined for the quenching analysis (Schreiber et al. 1986, Genty et al. 1989, van Kooten and Snel 1990):

$q_{\mathrm{P}}=\frac{F_{m}^{\prime}-F_{s}}{F_{m}^{\prime}-F_{o}^{\prime}}$

However, as first argued by Joliot and Joliot (1964), this relationship, at least in the presence of a PSII inhibitor like DCMU, is affected by the exchange of excitation energy between the antennae of different PSII RCs. This process has been called connectivity (e.g., Bennett 1983; Dau 1994; Lavergne and Trissl 1995) or grouping (Strasser 1978; Strasser et al. 2004). The parameter $q_{\mathrm{P}}$ is based on the socalled puddle model, which ignores the connectivity among PSII units. However, if connectivity also affects the whole fluorescence rise in the absence of inhibitors like DCMU, $q_{\mathrm{P}}$ needs a correction to take this process into account (Kramer et al. 2004b).

The question of how much connectivity affects the fluorescence rise is a contentious one. Strasser and Stirbet (2001) showed, on the basis of a simulation, that in the absence of DCMU connectivity mainly has a measurable effect on the first $300 \mu$ s of the fluorescence rise. Beyond that point re-opening and again closing PSII RCs and the effect of the different $S$ states on the fluorescence rise (see Questions 15 and 22) disturb the simple relationship that can be derived for DCMU-inhibited PSII RCs. Oja and Laisk (2012) demonstrated only a negligible effect of PSII connectivity and observed that $q_{\mathrm{P}}$ is proportional to the fraction of open PSII centers in the steady state. Tóth et al. (2007b) showed that the relation between $F_{J}$ and the area between the OJ rise and $F_{M}$ as a function of the extent of anaerobiosis was linear, indicating that at the $F_{J}$ step connectivity no longer affects the fluorescence rise. It has also been suggested that the sigmoidicity of the initial fluorescence rise, which is interpreted to be consequence of connectivity (reviewed by Stirbet 2013) can alternatively be explained on the basis of two overlapping exponential reactions (Vredenberg 2008; Schansker et al. 2011). This can account for the disappearance of the sigmoidicity of the fluorescence rise when the temperature is lowered to $-10{ }^{\circ} \mathrm{C}$ (Schansker et al. 2011). Schansker et al. $(2011,2014)$ provided experimental proof that only $70 \%$ of the variable fluorescence is related to the redox state of $Q_{A}$, introducing an additional complication. And finally, there is an important difference between the fluorescence rise in the absence and presence of a PSII inhibitor like DCMU. In the presence of an inhibitor, there is one single charge separation and all $Q_{A}$ becomes gradually reduced (as a function of the excitation rate). In the absence of an inhibitor, at, e.g., $3000 \mu \mathrm{mol}$ photons $\mathrm{m}^{-2} \mathrm{~s}^{-1}$, a single excitation of all reaction centers is complete after about $200 \mu \mathrm{s}$ (cf Neubauer and Schreiber 1987; Lazár and Pospísil 1999). At longer times, as mentioned above, there is a continuous re-oxidation and re-reduction of $Q_{A}$ in all RCs (see Questions 16 and 22), where the connectivity effect is likely to average out between all opening and closing PSII RCs.

The experimental data presented in the previous paragraph are not widely known and an approach based on the assumption that connectivity affects the whole fluorescence rise between $F_{O}$ and $F_{M}$ (Kramer et al. 2004b) is gaining wider acceptance. Kramer et al. (2004b) derived a modified parameter based on the "lake" model that considers the units to be fully connected, which they called $q_{\mathrm{L}}$ (Kramer et al. 2004b):

$q_{\mathrm{L}}=\frac{F_{m}^{\prime}-F_{\mathrm{s}}}{F_{m}^{\prime}-F_{o}^{\prime}} \cdot \frac{F_{o}}{F_{\mathrm{s}}}$

For the calculation of $q_{\mathrm{L}}$ without the measurement of $F_{O^{\prime}}$, Kasajima et al. (2009) derived the following equation:

$q_{\mathrm{L}}=\left(\frac{1}{F_{\mathrm{s}}}-\frac{1}{F_{m}^{\prime}}\right) /\left(\frac{1}{F_{o}}-\frac{1}{F_{m}}\right)$

With the parameters $q_{\mathrm{P}}$ or $q_{\mathrm{L}}$, we want to quantify the fraction of open PSII RCs, i.e., the fraction of PSII RCs with $Q_{A}$ in the oxidized state, in the light-adapted state (Kramer et al. 2004b; Roháček et al. 2008). Depending on the assumptions made (effect of connectivity or not), the value " $1-q_{\mathrm{P}}$ " or " $1-q_{\mathrm{L}}$ " represents the approximate redox state of $Q_{A}$, expressed as $Q_{A}^{-} / Q_{A}$ (tot) (Schreiber and Bilger 1987; Weis and Berry 1987). The expression " $1-q_{\mathrm{P}}$ " represents the balance between excitation rate 
and forward electron transport and is a reflection of the excitation pressure inside PSII (Ögren and Rosenquist 1992). It is also a measure for the degree of RC closure (Björkman and Demmig-Adams 1995; Roháček and Barták 1999).

$q_{\mathrm{P}}$ or $q_{\mathrm{L}}$ values vary between 0 and 1 , where 1 is observed in a fully relaxed dark-acclimated state (where $F_{S}=F_{O}$ ) and 0 represents the state when all $Q_{A}$ is reduced $\left(Q_{A}^{-}\right)$and $F_{S}=F_{M}{ }^{\prime}$. See Question 5 for a discussion of the relation between all $Q_{A}$ reduced and $F_{M}$.

An example of photochemical quenching $\left(q_{\mathrm{P}}, q_{\mathrm{L}}\right.$ and $\left.q_{\mathrm{L}}(c)\right)$ and $\Phi_{\mathrm{PSII}}$ as a function of the actinic light intensity is presented in Fig. 2a.

Figure 2 demonstrates that there is a considerable difference between $q_{\mathrm{P}}$ and $q_{\mathrm{L}}$, as well as between values of $q_{\mathrm{L}}$ calculated with or without $F_{O}{ }^{\prime}$. Figure $2 \mathrm{~b}$ illustrates that the relationship between $q_{\mathrm{P}}$ and $q_{\mathrm{L}}$ is nonlinear. It is worth mentioning that Pfündel et al. (2013) showed that $F_{O}{ }^{\prime}$ values estimated on the basis of the method of Oxborough and Baker (1997) are systematically too low (see Question $6)$.

The choice between $q_{\mathrm{P}}$ and $q_{\mathrm{L}}$ depends on the way we look at the Chl $a$ fluorescence induced by a saturating pulse. If we treat it as essentially a single charge separation, an analogy with the fluorescence rise in the presence of DCMU can be inferred, as Kramer et al. (2004b) did. If we take into account that the fluorescence rise induced by a saturating pulse consists of many turnovers of $Q_{A}$, the analogy is lost and then the straightforward parameter $q_{\mathrm{P}}$, though far from perfect, is probably a much better approximation of the $Q_{A}$ redox state in the light then the parameter $q_{\mathrm{L}}$.

\section{Question 10: Is the electron transport rate (ETR) calculated from $\mathrm{Chl} a$ fluorescence a reliable parameter?}

The electron transport rate (ETR) estimated from Chl $a$ fluorescence is often defined as:

$\mathrm{ETR}=\phi_{\mathrm{PSII}} \cdot \mathrm{PPFD} \cdot 0.5 \cdot$ leaf absorptivity coefficient

$$
\left(\mu \mathrm{mol} \text { electrons } \mathrm{m}^{-2} \mathrm{~s}^{-1}\right)
$$

where $\Phi_{\text {PSII }}$ (which is dimensionless) is the effective quantum yield of photosystem II in the light; PPFD ( $\mu$ mol photons $\mathrm{m}^{-2} \mathrm{~s}^{-1}$ ) is the photosynthetic photon flux density incident on the leaf (or any green organ); leaf absorptivity coefficient (which is dimensionless) is the absorptance of the photosynthetic organ, i.e., the proportion of the incident PPFD effectively absorbed by the photosynthetic surface, and " 0.5 " is a correction factor for PPFD, assuming that half of the photons are absorbed by PSI and the other half by PSII as first formulated by Krall and Edwards (1992). As formula 5 shows, ETR and $\Phi_{\mathrm{PSII}}$ are proportional and are, therefore, closely related parameters.

The parameter ETR has been shown to correlate well with linear electron flow calculated on the basis of $\mathrm{O}_{2}$ evolution rates (Flexas et al. 1999; von Caemmerer 2000). Genty et al. (1989) observed a linear correlation between $\Phi_{\text {PSII }}$ and $\mathrm{CO}_{2}$ assimilation rate at $1 \% \mathrm{O}_{2}$ for barley and $20 \% \mathrm{O}_{2}$ for mays. Edwards and Baker (1993) extended the number of conditions under which a linear correlation was observed. However, in many other studies a nonlinear, somewhat concave, relationship was observed (Peterson et al. 2001 and references therein). Correcting for PSI
Fig. 2 Photochemical quenching. a The parameters $\Phi_{\mathrm{PSII}}, q_{\mathrm{P}}, q_{\mathrm{L}}$ and $q_{\mathrm{L}}(\mathrm{c})$ as a function of the actinic light intensity determined on wheat leaves. b The relationship between $q_{\mathrm{P}}$ and $q_{\mathrm{L}}$ is nonlinear especially at low light intensities (values close to 1), whereas the relationship between $q_{\mathrm{L}}$ and $q_{\mathrm{L}}(\mathrm{c})$ is linear with $q_{\mathrm{L}}(\mathrm{c})$ systematically lower than $q_{\mathrm{L}}$ (Živčák and Brestič, unpublished data)
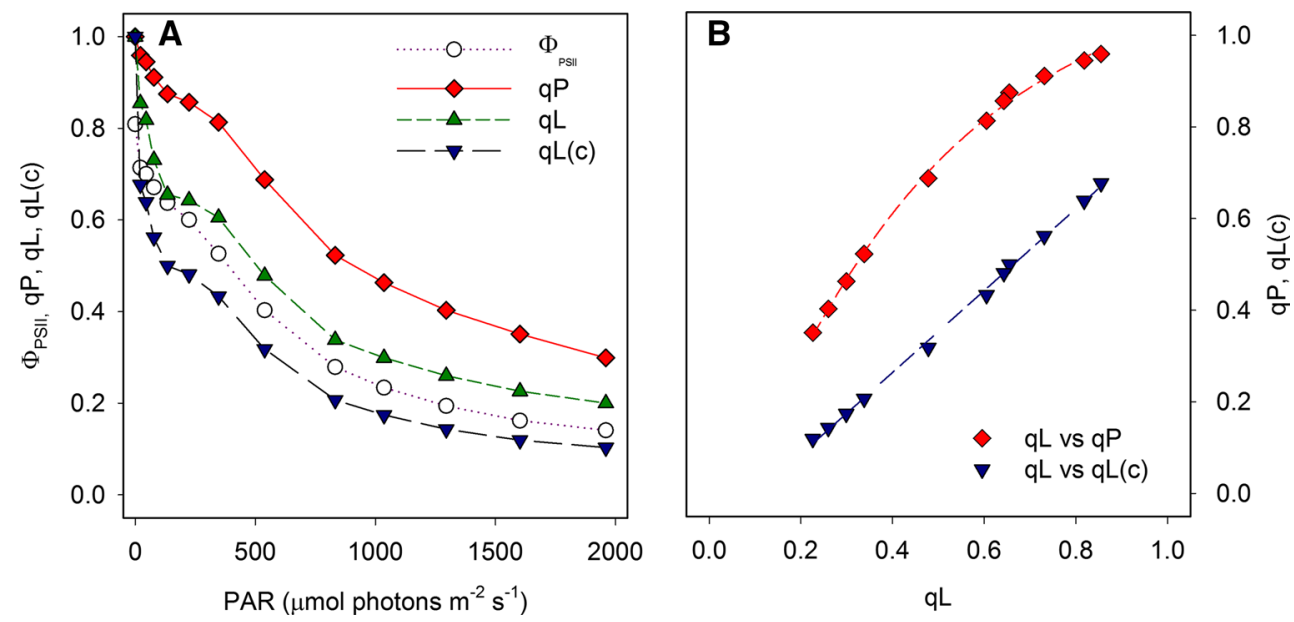
fluorescence improves the linearity of the relationship (Peterson et al. 2001).

For the leaf absorptivity coefficient, 0.85 is a typical value for C3 plants (Ehleringer and Pearcy 1983; Krall and Edwards 1992; Schultz 1996).

The value " 0.5 " is a rough estimate. Von Caemmerer (2000) wrote that this factor varies between 0.45 and 0.5 . In contrast, in some studies (e.g., Strasser and Butler 1977) it was observed that PSII absorbs more light than PSI. As a first approximation, and in the absence of further information, " 0.5 " is likely the best choice.

Peterson and Havir $(2003,2004)$ considered the possibility that the rate constants $\mathrm{kN}$ of heat dissipation and/or $\mathrm{kF}$ of $\mathrm{Chl} a$ fluorescence change during the OJIP fluorescence rise. According to Dau (1994) the relationship between $1 / F_{O}$ and $1 / F_{M}$ should be linear and proportional (slope $=1$ ) if NPQ is purely due to dissipation of excitation energy in the antenna. Peterson and Havir (2003) tested this assumption for WT and psbS mutants of Arabidopsis thaliana. They observed that for WT leaves the relationship is biphasic with a steeper slope at low light intensities than at high light intensities. In the case of the psbS-mutant leaves there is a strong deviation from linearity, mainly because the mutant shows no $F_{O}$ quenching. Peterson and Havir (2004) extended this study to $10 \mathrm{~A}$. thaliana lines and concluded that during an OJIP rise (saturating pulse) the $\mathrm{kN}$ and/or $\mathrm{kF}$ changes. They showed that this affects the relationship between ETR based on fluorescence measurements and ETR based on gas exchange measurements. They further showed that the relationship between these two parameters could be improved considering the redox state of $Q_{A}$. Schansker et al. $(2011,2014)$ proposed that during the OJIP rise the fluorescence yield increases ( $\mathrm{kF}$ increases) depending on the time $Q_{A}$ remains reduced, before becoming re-oxidized by forward electron transport. This interpretation model supports the observations and interpretations by Peterson and Havir (2003, 2004).

There are several other factors that may affect the correction factor 0.5: (1) state transitions (see Question 8) can cause a redistribution of light between PSII and PSI on a minutes timescale, especially in algae (Bonaventura and Myers 1969; Depège et al. 2003; Iwai et al. 2008); (2) the extent of stacking and the associated changes in spillover, again especially in algae (see Trissl and Wilhelm 1993 for a discussion of this point), may have a considerable effect on the distribution of light between the two photosystems; (3) as shown by Anderson et al. (1988), there are at least threefold differences in the PSII/PSI ratio (ranging from at least 1:1 to 1:3) between different plant species. This range may in part be compensated by differences in PSII antenna size, but it is likely that it also represents some variability in this parameter; (4) long-term acclimation of a plant species to different light regimes affects PSII antenna size and PSII/PSI ratio (Bailey et al. 2001; Ballottari et al. 2007; Hogewoning et al. 2012; Bielczynski et al. 2016). A change in both the PSII antenna size and the PSII/PSI ratio affects the $\mathrm{Chl} a / b$ ratio and may be used as an indicator for effects related to points (3) and (4).

Not only variations in the value " 0.5 ," but also corrections of $\Phi_{\mathrm{PSII}}$ should be considered. As noted in Question 6, a correction of $\Phi_{\mathrm{PSII}}$ for PSI fluorescence emission will yield a more reliable ETR value, especially for $\mathrm{C} 4$ plants, and improve the linear correlation with $\mathrm{CO}_{2}$ assimilation measurements (Pfündel et al. 2013).

In Kalaji et al. (2014a) simultaneous Chl $a$ fluorescence and $\mathrm{CO}_{2}$ assimilation measurements, and the information such measurements can yield, are discussed. In that paper the problems of using $\Phi_{\text {PSII }}$ or ETR as indicators for the quantum yield of $\mathrm{CO}_{2}$ assimilation by the leaf $\left(\Phi_{\mathrm{CO}_{2}}\right)$ were also discussed extensively. By determining the linearity of the relationship between ETR and $\mathrm{CO}_{2}$ assimilation (in the case of $\mathrm{C} 3$ plants in the presence of $2 \% \quad \mathrm{O}_{2}$ to suppress photorespiration), the usefulness of ETR as a measure for $\mathrm{CO}_{2}$ assimilation can be established for individual cases.

In C3 species where the linearity between ETR and net $\mathrm{CO}_{2}$ assimilation is often absent due to the existence of alternative electron sinks, especially photorespiration (see Question 11), a multivariate approach was shown to be a good alternative (Losciale et al. 2015). The rationale of this method is: to consider the main factors affecting net photosynthesis; to identify the related variables, and to combine these variables using a multivariate semi-mechanistic approach. Roughly, net photosynthesis $\left(P_{\mathrm{n}}\right)$ is a function of: (1) the electron transport rate of the ETC; (2) the $\mathrm{CO}_{2}$ concentration at the carboxylative sites, which depends on stomatal and mesophyll conductance; and (3) the carboxylative activity of Rubisco, which depends on the Michaelis-Menten constants for carboxylation, Kc, and photorespiration, Ko. The first factor can easily be determined using Chl $a$ fluorescence (ETR), and the last two are strictly related to the leaf-to-air temperature difference $(\Delta T)$ and the leaf temperature $(\mathrm{Tl})$, used for Ko and $\mathrm{Kc}$ estimation (von Caemmerer 2000). Using the function

$P_{\mathrm{n}}=\alpha+\beta_{1}\left[\operatorname{ETR}\left(\frac{\mathrm{Ko}}{\mathrm{Kc}}\right)\right]+\beta_{2}(\Delta T)$

it was possible to estimate accurately net photosynthesis based on the measurements of ETR, leaf and air temperature, only. The parameters $\alpha, \beta_{1}$ and $\beta_{2}$ are species-specific, and the model has been parameterized and validated for apple and pear (Losciale et al. 2015). As illustrated in Fig. 3, the calculated $P_{\mathrm{n}}$, called $I_{\mathrm{PL}}$ in Fig. 3b, shows a better linear relation with $P_{\mathrm{n}}$ than $J_{\mathrm{PSII}}$ (Fig. 3a). 

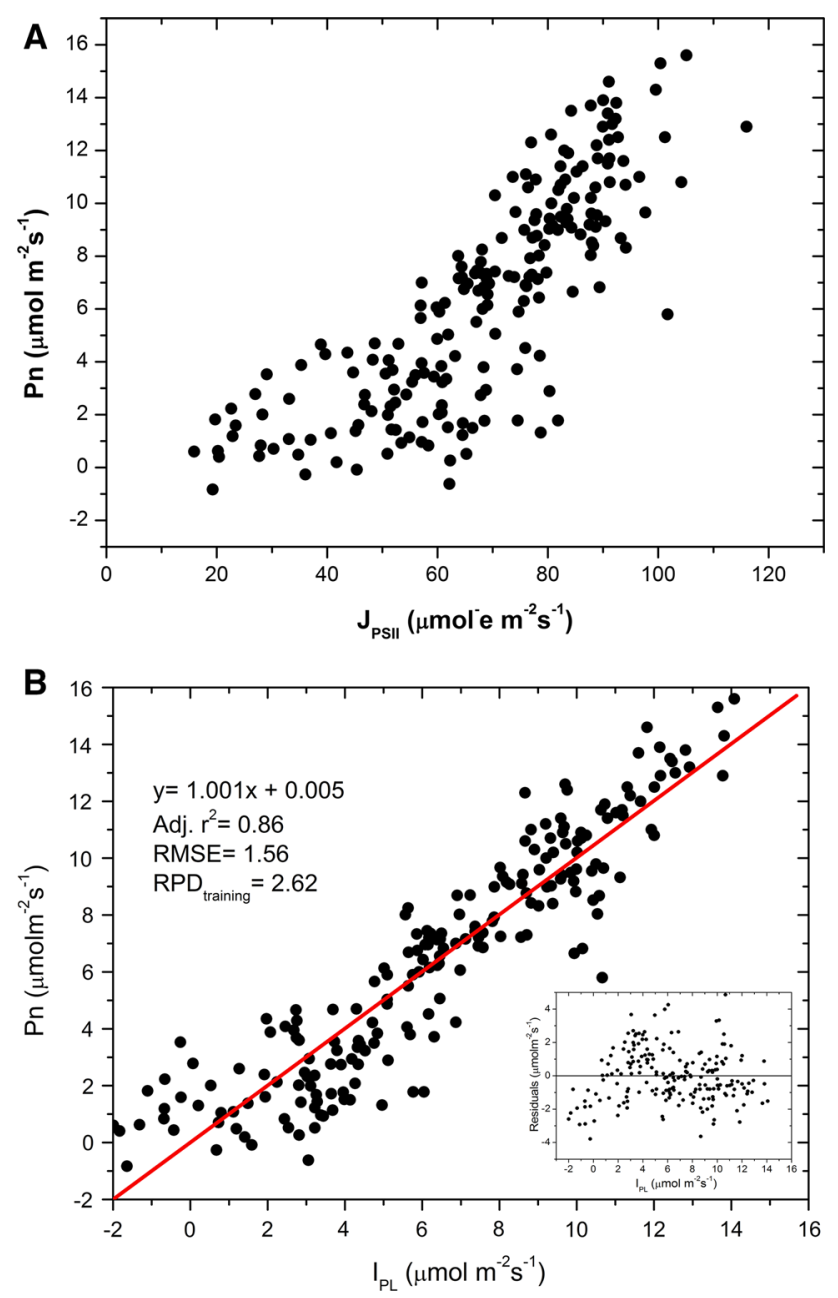

Fig. 3 Factors affecting the relationship between the electron transport rate (ETR) and net photosynthesis $\left(P_{\mathrm{n}}\right)$ in apple. a $P_{\mathrm{n}}$ as a function of ETR for 21 apple (C3 plant) genotypes exposed to different drought stress conditions. The relation is nonlinear; $\mathbf{b} P_{\mathrm{n}}$ as a function of a parameter $\left(I_{\mathrm{PL}}\right)$ based on ETR, leaf temperature and the leaf-to-air temperature difference and derived by a multivariate approach. This yielded a strongly linearly correlated relationship with a slope of nearly 1. (Figures $\mathbf{a}$ and $\mathbf{b}$ are adapted from Figs. 1d and 3 in Losciale et al. 2015)

In summary, ETR has been shown to linearly correlate with $\mathrm{CO}_{2}$ assimilation under several conditions. $\mathrm{CO}_{2}$ assimilation is, of course, only one of the available electron sinks (see Question 11), photorespiration in C3 plants being an important alternative sink, and, therefore, it should not be used to estimate absolute rates of $\mathrm{CO}_{2}$ assimilation (Baker 2008). Correcting the ETR calculation for PSI fluorescence emission (Question 6), taking into account possible deviations of the factor from 0.5 as well as the inclusion of several easy to measure parameters as described in the previous paragraph may further improve the usefulness and reliability of this parameter.

\section{Question 11: What are the experimental differences between $\mathrm{Chl} \boldsymbol{a}$ fluorescence and gas exchange measurements?}

ETR ("Electron Transport Rate") is the fluorescence parameter that gives a measure for the linear transport of electrons from $\mathrm{H}_{2} \mathrm{O}$ (i.e., from PSII) to the Calvin-Benson cycle (or other sinks, see below) under steady-state conditions. If ETR and the $\mathrm{CO}_{2}$ assimilation rate are compared, several theoretical and experimental factors have to be considered.

\section{Electron sinks}

It is important to realize that $\mathrm{CO}_{2}$ assimilation is only one of several potential electron sinks. The most important competitor under stress conditions, that cause reduced stomatal opening, is photorespiration. Photorespiration is mainly an issue for C3 plants (Cornic and Fresneau 2002). The most important alternative pathway under high light conditions is also photorespiration (Foyer and Noctor 2009; Bauwe et al. 2010). Photorespiratory activity can be nearly completely suppressed if the oxygen concentration is reduced to $2 \%$ or less. In $\mathrm{C} 4$ plants photorespiration is negligible (Laisk and Edwards 1998). Other electron sinks are the Mehler reaction (i.e., the reduction of molecular oxygen on the acceptor side of PSI) (Asada 1999; Foyer and Noctor 2009), cyclic electron transport around PSI (e.g., Heber and Walker 1992), nitrogen and sulfur metabolism, which also consume ATP and NADPH (e.g., Neyra and Hageman 1974; Leustek et al. 2000; Kopriva and Rennenberg 2004) and the export of reducing equivalents to mitochondria or peroxisomes (Raghavendra and Padmasree 2003; Yoshida et al. 2007). The importance of these alternative sinks under steady-state conditions has been a discussion issue for many, many years (see, e.g., Peterson and Havir 2004) and is beyond the scope of the present review.

\section{Structural considerations}

The assimilation rate of $\mathrm{CO}_{2}$ reflects the photosynthetic activity of the whole leaf, whereas ETR measurements derive mainly from fluorescence emission by chloroplasts in the top cell layers (in most cases, mainly the palisade parenchyma cells) of the leaf. In addition, $P_{\mathrm{n}}$ is measured by infrared gas analyzers (IRGAs) on an entire leaf or, more commonly, a significant part of it; Chl $a$ fluorescence, in contrast, is measured on a much more limited area of the leaf (a few square millimeters) (Rosenqvist and van Kooten 2003). Thus, the chloroplasts placed near the adaxial side of the leaf may be photoinhibited (e.g., by strong light or 
low temperatures in combination with moderate light), whereas other chloroplasts (deeper in the leaf) are photosynthesizing normally. However, given the steep light gradient inside the leaf, these chloroplasts will receive much less light.

In summary, IRGA and Chl $a$ fluorescence refer to different spatial scales in terms of surface and depth. Discrepancies between ETR and $\mathrm{CO}_{2}$ assimilation can, at least in part, be explained on the basis of these differences.

Finally, coexistence of mitochondrial respiration and photosynthetic metabolism needs to be considered. Photosynthesis measured by IRGA represents the net exchange of $\mathrm{CO}_{2}$ - gross photosynthesis minus the $\mathrm{CO}_{2}$ produced by respiration and photorespiration. In general, the respiration rate of a leaf is low compared with photosynthesis (around 5-10\%) (e.g., Kromer 1995) and can therefore be disregarded. However, in leaves with very low photosynthetic rates (e.g., plants under severe environmental stress or deep-shade-adapted species), respiration becomes comparatively more important.

If a linear relationship between ETR and $\mathrm{CO}_{2}$ assimilation is observed, one can conclude either that the contribution of alternative sinks is negligible, or that their contribution is light intensity independent. The same is true for the structural effects. A systematic study of the effects of these factors on the relationship between ETR and $\mathrm{CO}_{2}$ assimilation would, therefore, be useful. It is, however, always good to keep in mind the factors mentioned above that may affect the relationship between fluorescence and IRGA measurements.

\section{Question 12: Is it meaningful to determine energy partitioning?}

Demmig-Adams et al. (1996) published a paper in which they observed that the parameters $q_{N}$ and NPQ only gave relative values for energy dissipation as heat. They wanted to find a way to quantify in absolute terms the fraction of energy dissipated as heat. The authors noted that in sun leaves non-photochemical quenching was high and the PSII reaction centers remained relatively open. In shade leaves little non-photochemical quenching was induced and the PSII reaction centers were to a large extent closed. The authors then concluded that in shade leaves there is little heat dissipation. This conclusion, however, is questionable.

Let's have a look at a dark-adapted leaf. If a saturating pulse is given to a dark-adapted leaf, all PSII reaction centers become closed, photochemistry is reduced to 0 , and excitation energy can only be dissipated as either heat or fluorescence emission (if we ignore connectivity, spillover and oxidative damage). For such a saturating pulse it is also assumed that it does not induce non-photochemical quenching. Under such conditions fluorescence emission has been determined to be $\sim 10 \%$ (Barber et al. 1989). This means that at least $90 \%$ of the excitation energy in closed PSII reaction centers is dissipated as heat. This happens both in sun and in shade leaves. The induction of $q_{\mathrm{E}}$ can increase this percentage by no more than $10 \%$ by completely outcompeting fluorescence emission. In other words, the induction of the processes associated with $q_{\mathrm{E}}$ has only a rather small effect on the fraction of excitation energy dissipated as heat. However, the purpose of the processes associated with $q_{\mathrm{E}}$ is not so much to increase the fraction of heat dissipation; its purpose is to reduce the lifetime of excitation energy in the antenna. It may be expected that the probability that excitation energy will cause oxidative damage is a function of the lifetime of an exciton.

It can, therefore, be argued that the idea of DemmigAdams et al. (1996) was based on a false premise. Dissipation of excitation energy as heat in closed PSII reaction centers is always $90 \%$ or higher. What changes is an increase of the efficiency with which the antenna can dissipate excitation energy as heat if processes associated with $q_{\text {E }}$ are induced. A shift in the lifetime of excitation energy from $1.6-1.8$ to $0.5 \mathrm{~ns}$ as violaxanthin is turned into zeaxanthin $(\mathrm{Z})+$ antheraxanthin $(\mathrm{A})$ has been observed by Gilmore et al. (1998). These authors observed that changes in the parameter $F_{M}^{\prime} / F_{M}$ can be used to monitor directly fluorescence lifetimes, intrathylakoid $\mathrm{pH}$ and $[\mathrm{Z}+\mathrm{A}]$.

Recently, Lazár (2015) reviewed all the efforts that have been made to improve on the original concept of DemmigAdams et al. (1996). Demmig-Adams et al. argued that in addition to photosynthesis and dissipation there is an undefined component, called "excess" by the authors. Since no process is associated with this component this is difficult to accept and understand (see Lazár 2015 for a discussion of this point). Another point of criticism of the approach of Demmig-Adams et al. can be that their approach is too simplistic and ignores other processes that affect Chl $a$ fluorescence. Kornyeyev and Hendrickson (2007) and Kornyeyev et al. (2013) included photoinhibition and other photochemical and non-photochemical processes in their analysis. However, the observation that only $\sim 10 \%$ of the absorbed energy, which in dark-adapted leaves is emitted as fluorescence, can be quenched and emitted as heat is also valid for all these improvements.

In summary, fluorescence, heat dissipation and photosynthesis compete with each other. In a closed PSII RC photosynthesis is 0 and only fluorescence and heat dissipation compete. If the rate constant for heat dissipation $(\mathrm{kN})$ increases, heat dissipation will increase at the cost of fluorescence emission, which is observed as fluorescence quenching. The rate constant $\mathrm{kN}$ increases if the lifetime of the associated process decreases as observed by Gilmore et al. (1998) for the xanthophyll cycle. 


\section{Question 13: What is the fate of light absorbed by PSII?}

Once light is absorbed by the PSII antenna, it is efficiently transferred to the PSII RCs and if they are in the open state the most likely fate of the excitation energy $(\sim 90 \%)$ is that it is used to drive a charge separation between the accessory chlorophyll $\mathrm{Chl}_{\mathrm{D} 1}$ and Pheo followed by a transfer to $Q_{A}$ (see Question 6). Long and Humphries (1994) reported on the basis of the literature that in full sunlight the percentage of absorbed light used for the photosynthetic process as a whole drops to $5-10 \%$. This observation was confirmed by Losciale et al. (2010).

Alternative de-excitation pathways are: (1) dissipation as heat by the carotenoids bound to the antenna proteins (Gibasiewicz et al. 2005; Bode et al. 2009); (2) fluorescence emission by Chl $a$ molecules (Butler 1978; Lazár 1999; Maxwell and Johnson 2000; Kalaji et al. 2012b); (3) in the case of photoinhibition of a part of the PSII RCs, transfer of excitation energy from active to inactive RCs (see below); (4) the excitation of oxygen, resulting in singlet oxygen (reviewed in Krieger-Liszkay et al. 2008). These processes are all in competition. If $Q_{A}$ is reduced, the pathway related to charge separation is blocked, the lifetime of the excitation energy increases, and the other deexcitation pathways become more important.

The rate constant for dissipation as heat is increased considerably by photoprotective quenching, associated with $\Delta \mathrm{pH}$, PsbS and the xanthophyll cycle (Ort 2001). The xanthophyll cycle (i.e., the formation of zeaxanthin) can reduce fluorescence emission by 75-90\% (DemmigAdams et al. 1996; Flexas and Medrano 2002), which means that nearly all absorbed energy is dissipated as heat (see also Question 12).

Pogson et al. (1998) found that in addition to the xanthophyll cycle pigments (zeaxanthin and antheraxanthin), the $\alpha$-carotene-derived xanthophyll lutein, which is a structural component of the subunits of the light-harvesting complexes, contributes to the dissipation of excess absorbed light energy and the protection of plants against photooxidative damage. Lutein is also thought to contribute to $\mathrm{q}_{\mathrm{E}}$ (Pogson et al. 1998; Müller et al. 2001). The xanthophyll neoxanthin, on the other hand, that also binds to antenna proteins, does not seem to play a role in energy dissipation (reviewed by Jahns and Holzwarth 2012).

A special case, in this respect, is formed by photoinactivated PSII RCs. When inactivated, PSII RCs are disassembled and repaired (Aro et al. 1993). During a clear sunny day, in the absence of any additional stress, the total PSII pool can be completely inactivated and repaired without photoinhibition being detectable (Chow and Aro 2005). If plants also suffer from other stresses like water or nitrogen deficiency (Jifon and Syvertsen 2003; Cheng et al. 2000), a significant population of inactivated PSII RCs may accumulate in the thylakoid stacks. It has been proposed that these RCs play a role in the dissipation of light energy under high light conditions (Matsubara and Chow 2004; Chow et al. 2012). Matsubara and Chow (2004) showed that photoinhibition of PSII enriched membranes, which do not show xanthophyll cycle activity, caused the induction of a 0.3-ns lifetime component at the expense of 1.7- and 3.9-ns components. This suggests that photoinhibited PSII RCs become strong quenchers of light energy. On this basis, Matsubara and Chow (2004) proposed that by connectivity with other active PSII RCs the photoinhibited PSII RCs can act as quenching sinks. In thylakoid membranes isolated from photoinhibited leaves the authors observed 1.25- and 0.58-ns lifetime components, which they associated with photoinhibition. It may be noted, however, that the assignment of the 0.58-ns lifetime component to photoinhibition can be challenged, since Gilmore et al. (1998) ascribed a similar 0.5-ns component to the effect of zeaxanthin formation.

The formation of singlet oxygen by excited Chl molecules is a multistep process. The excited singlet $\mathrm{Chl}$ state $\left({ }^{1} \mathrm{Chl}\right)$ can return to the ground state under the emission of fluorescence. Alternatively, a transition of the ${ }^{1} \mathrm{Chl}$ state to the triplet state $\left({ }^{3} \mathrm{Chl}\right)$ can occur with a certain probability. There are two sources of ${ }^{3} \mathrm{Chl}$ : the PSII antenna and ${ }^{3} \mathrm{P} 680$ following recombination (Krieger-Liszkay 2004). ${ }^{3} \mathrm{Chl}$ can return to the ground state under transfer of its energy to $\mathrm{O}_{2}$. This leads to the formation of very reactive singlet $\mathrm{O}_{2}$ $\left({ }^{1} \mathrm{O}_{2}\right)$. The recombination pathway depends on the midpoint potential of $Q_{A}^{-}$. Fufezan et al. (2002) showed that in the presence of the phenolic herbicide bromoxynil the midpoint potential of $Q_{A}^{-}$is lowered, charge recombination between Pheo ${ }^{-}$and $\mathrm{P}_{680}{ }^{+}$is favoured, and the ${ }^{1} \mathrm{O}_{2}$ yield is higher. This indicates that it is this recombination pathway that leads in the RC to singlet oxygen formation via the ${ }^{3} \mathrm{P} 680$ state. Singlet $\mathrm{O}_{2}$ is thought to play a role in photoinhibition (see Krieger-Liszkay et al. 2008 for a discussion of this point).

The excitation pressure is not only reduced by more efficient heat dissipation but also by a higher electron transport rate (see Question 11). As discussed in Question 8 , preventing a strongly reduced acceptor side of PSI is important. Tiwari et al. (2016) showed recently for the pgr5 mutant, in which cyclic electron transport around PSI is inhibited, that the FeS clusters on the acceptor side of PSI become more easily damaged under high light conditions, showing the importance of this process for the protection of PSI. The glutathione-ascorbate cycle is also thought to play a role in the protection of the acceptor side of PSI from damage under conditions of low Calvin-Benson cycle 
activity (Noctor and Foyer 1998; Baker and Rosenqvist 2004). Baker and Rosenqvist (2004) noted that the particular physiological effects of different stresses determine which alternative process becomes more active.

Regulation of thermal and photochemical de-excitation pathways, together with the PSII recovery system, all contribute to a photoprotective system, which prevents photodamage to the photosynthetic apparatus (Baker and Rosenqvist 2004). Photoinhibition and photoprotective mechanisms can be detected using several techniques, but the most useful method is the measurement of Chl $a$ fluorescence (Krause and Weis 1991; Govindjee 1995; Maxwell and Johnson 2000; Losciale et al. 2008, 2010).

\section{Question 14: How to probe the donor side of PSII?}

Oxygen evolution takes place at the donor side of PSII, which consists of a Mn cluster of four manganese ions, a $\mathrm{Cl}^{-}$and $\mathrm{Ca}^{2+}$ ion and the surrounding protein environment (Debus 1992; Yocum 2008). Access of reductants like ascorbate is limited by the presence of three extrinsic proteins of the oxygen-evolving complex (OEC), i.e., PsbO, PsbP and PsbQ (reviewed by Tóth et al. 2013). The electron transfer link between the Mn cluster and P680 is a tyrosine molecule located in the D1 protein called TyrZ. During the oxygen-evolving process, the PSII donor side passes through five redox states, $S 0$ to $S 4$, of which the $S 4$ state is not stable and passes to the $S 0$ state within about 2 ms under the release of an oxygen molecule. Flash-induced oxygen evolution (Kok et al. 1970; Joliot et al. 1971), flash-induced fluorescence (Bouges-Bocquet 1980) and flash-induced DF (Grabolle and Dau 2005) are characterized by period-4 oscillations. For the analysis of the $S$ states, the five-state model as introduced by Kok et al. (1970) is still largely valid: The OEC following dark adaptation is in the $S 1$ state, and the period-4 oscillations are dampened by misses and double hits, i.e., a flash induces two charge separations [see, e.g., Dau et al. (2012) for a more mechanistic model for the period-4 oscillations]. The only major change is that a population of about $25 \%$ RCs, which Kok et al. ascribed to PSII RCs in the $S 0$ state in darkness, is now ascribed to electron donation by reduced TyrD to the Mn cluster between flashes (Velthuys and Visser 1975; Vermaas et al. 1984; Shinkarev 2005). Donation of an electron by TyrD to the Mn cluster is a slow reaction and can, in continuous light, not compete with the light-induced turnover of the OEC. In dark-adapted samples all RCs can be considered to be in the $S 1$ state. Period4 oscillations can be used to analyze the $S$ state distribution of a sample (cf. Ioannidis et al. 2000). This analysis can even be extended to super-reduced states such as the $S-1$ and $S$-2 states (Schansker et al. 2002). Period-4 oscillation in the $F_{O}$ level can be used to follow the decay kinetics of the $S$ states under natural conditions, but also in response to external electron donors like hydroxylamine or NO (see Ioannidis et al. 2000). The time resolution of such measurements depends on the flash frequency, which is, in practice, around $10 \mathrm{~Hz}$ (one flash every $100 \mathrm{~ms}$ ). This type of measurement can also be carried out with flash illumination of a sample on a bare oxygen electrode (e.g., Messinger and Renger 1990, 1993). The disadvantage of a bare oxygen electrode is that a rapid injection and mixing of reactants is not possible (Messinger and Renger 1990). Period-4 oscillations are usually measured on PSII enriched membranes, thylakoid membranes or algal cells. David Kramer and coworkers introduced, in 1990, an instrument to measure period-4 oscillations on leaves in the field (Kramer et al. 1990), although few studies using this instrument have been published.

In response to heat stress, the Mn cluster can be destroyed. Heat stress can cause a dissociation of extrinsic proteins, followed by a super-reduction of the Mn cluster, which destabilizes it, resulting in a disintegration of the Mn cluster and a release of the Mn ions into the lumen (Yamane et al. 1998; Pospícil et al. 2003; Barra et al. 2006). In the case of severe heat stress, the OJ rise turns into a $\mathrm{K}$ peak (e.g., Srivastava et al. 1997; Fig. 4). It has been proposed that the $\mathrm{K}$ peak, that can be made visible by subtraction of the double-normalized OJ rise, can be used

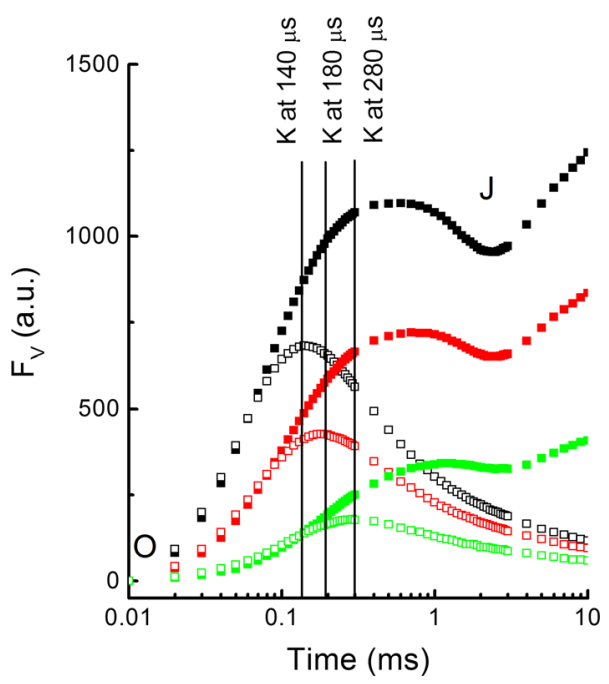

Fig. $4 \mathrm{Chl}$ a fluorescence transients of untreated (closed symbols) and severely high-temperature-stressed (open symbols) barley leaves illuminated with 5000 (green symbols), 10,000 (red symbols) and 15,000 (black symbols) $\mu \mathrm{mol}$ photons $\mathrm{m}^{-2} \mathrm{~s}^{-1}$. To allow a comparison of the kinetics the transients were shifted to 0 to have for all transients the same $O$ value. The time needed to reach the $\mathrm{K}$ peak at the different light intensities is indicated. The $\mathrm{K}$ peak equates approximately 1 charge separation in all $\mathrm{RCs}$ after which a shortage of electrons that can be donated by the donor side occurs, and the fluorescence intensity decreases due to re-oxidation of $Q_{A}^{-}$reduced by the first charge separation (modified from Tóth et al. 2007a) 
as a probe of PSII RCs with an inactive donor side (e.g., Smit et al. 2009; Yusuf et al. 2010). However, as discussed in Kalaji et al. (2014a), this is tricky, because the same phenomenon is also induced by differences in the PSII antenna size between samples and a difference in the redox state of the PQ pool (Strasser et al. 2001). There are, however, two alternative approaches available to researchers. At approximately $3000 \mu \mathrm{mol}$ photons $\mathrm{m}^{-2}$ $\mathrm{s}^{-1}$ one charge separation in all reaction centers takes about $200 \mu$ s (K step). PSII RCs with an inactive donor side are capable of a single charge separation and during this time interval the fluorescence rise is the same in active and inactive RCs (Tóth et al. 2007a; Fig. 4). Subsequently, TyrZ is re-reduced with a half-time of about $30 \mathrm{~ms}$ (Tóth et al. 2007a, 2009) and during this time no second stable charge separation can occur. This means that the $K$ to $J$ rise is slowed down as a function the fraction of PSII RCs with an inactive donor side. Making use of this phenomenon, the parameter $F_{K} / F_{J}$ was introduced to probe the extent of inactivation of the PSII donor side (Srivastava et al. 1995; Lu and Zhang 1999). Making use of the difference in the regeneration time of the donor side, it is also possible to give two strong 5-ms pulses spaced $2.3 \mathrm{~ms}$ apart (the dark interval when the pulse interval of the HandyPEA is set to 0). The ratio of the fluorescence intensity at $300 \mu$ s of the second and the first pulse can then be used as a measure of the fraction PSII RCs with an inactivated donor side (Oukarroum et al. 2009; see this paper also for a discussion of the different approaches).

In summary, to probe the fraction of PSII RCs with an inactive donor side, it is possible to make use of the difference in the regeneration time of the donor side of PSII (the re-reduction of $\mathrm{P}_{680^{+}}$and $\mathrm{TyrZ}^{+}$).

\section{Question 15: What is the character of the J step?}

The $\mathrm{J}$ step $\left(F_{2 \mathrm{~ms}}\right.$ or $\left.F_{3 \mathrm{~ms}}\right)$ forms the central point in the JIP test analysis. Giving the $\mathrm{J}$ step such a central role was one of the major innovations introduced by Strasser and Strasser (1995) together with the consistent use of a logarithmic time base to make the different steps of the OJIP rise visible. The J step of Strasser and coworkers equates the I1 step of Schreiber (Schreiber 1986; Neubauer and Schreiber 1987; Schreiber and Neubauer 1987). Compare for this equivalence panels A and B of Fig. 1 in Lazár (2006). In publications about the analysis of OJIP transients the time point taken for the $\mathrm{J}$ step is either at 2 or at $3 \mathrm{~ms}$ (e.g., Strasser and Strasser 1995 vs. Tóth et al. 2007b). Stirbet and Govindjee (2012) argued that it had to be $2 \mathrm{~ms}$ because this was the time point chosen by Strasser and Strasser (1995) for the JIP test. Kinetically, the choice is important. At low light intensities or for PSII reaction centers with a smaller antenna size the time needed to reach the $\mathrm{J}$ step is more than $2 \mathrm{~ms}$, and this provides additional variability, which may be interesting for a stress test. For people interested in the kinetics of the photosynthetic electron transport chain, the 3-ms point is the more logical choice. It is at the end of the step, where the traffic jam of electrons induced by the rate-limiting step presented by the exchange of reduced $\mathrm{PQH}_{2}$ for $\mathrm{PQ}$ is maximal. As shown in the literature, this time point remains the end of the $\mathrm{J}$ step independent of the light intensity; lowering the light intensity gradually makes $F_{3 \mathrm{~ms}}$ disappear (Schansker et al. 2005, 2011).

Another phenomenon observed, when using very high light intensities, is a dip occurring around 2-3 ms (Neubauer and Schreiber 1987; Schansker et al. 2005, 2011). A possible explanation for this dip is the presence of $\mathrm{P} 60^{+}$, which acts as an even stronger fluorescence quencher than $Q_{A}$ (Steffen et al. 2005; see Question 22). The lifetime of $\mathrm{P} 680^{+}$is short for all $S$ states, with the exception of the transition from the $S 3$ to $S 4$ state. As the light intensity is increased, the turnover of PSII will remain more synchronized during the first turnovers and the peak concentration accompanying the $S 3$ to $S 4$ state transition will not only occur at shorter times, but will also reach a higher peak value causing a stronger and more localized quenching effect.

It has been assumed that the $\mathrm{J}$ step is due to the rate limitation caused by the exchange of $Q_{B} \mathrm{H}_{2}$ for PQ (cf. Petrouleas and Crofts 2005; Schansker et al. 2005; Tóth et al. 2007b). The role of $\mathrm{P} 680^{+}$quenching discussed above suggests that the transitory accumulation of $\mathrm{P} 60^{+}$, in going from the $S 3$ to the $S 4$ state (after 3 charge separations), is the physical reason for the existence of the $\mathrm{J}$ step. For dark-adapted leaves and continuous light both processes occur synchronously. Using a preflash protocol, a desynchronization between PSII donor and acceptor sides can be induced. This happens following two preflashes. This creates the $S 3 Z \mathrm{ZP} 680 Q_{A} Q_{B}^{-}$state, where the $S 3$ state is quite stable during a, e.g., $100-\mathrm{ms}$ flash interval and $Q_{B} \mathrm{H}_{2}$ is exchanged within $2 \mathrm{~ms}$ for PQ. If $100 \mathrm{~ms}$ after the two preflashes the OJIP transient is measured, the induction kinetics of the fluorescence rise are quite drastically changed (Schreiber and Neubauer 1987; Strasser and Strasser 1998).

\section{Question 16: What is the information that can be derived from the Area parameter?}

Joliot and Joliot (1964) published evidence that the relationship between variable fluorescence and $Q_{A}$ redox state in the presence of an inhibitor (e.g., DCMU) that prevents the re-oxidation of $Q_{A}^{-}$is nonlinear. The authors proposed 
that this nonlinearity was due to the exchange of excitation energy between different PSII antennae. As a consequence the relationship became sigmoidal. Two years later, Malkin (1966) and Murata et al. (1966a) came up with an alternative. They proposed that the complementary area between $F_{M}$ and the fluorescence transient measured in the presence of an inhibitor of re-oxidation of $Q_{A}^{-}$is linearly related to the $Q_{A}^{-}$concentration. This was confirmed experimentally by Bennoun and Li (1973). Although this idea can still be applied, several complications have been identified.

First, the complementary area of fluorescence induction curves measured in the presence of DCMU was shown to consist of several rise components. Doschek and Kok (1972) concluded that the complementary area represented a two-electron process. Melis and Homann (1975, 1976) interpreted this phenomenon to represent PSII heterogeneity: PSII alpha and beta centers differing in antenna size. Subsequently, two more phases were identified, designated gamma and delta (e.g., Sinclair and Spence 1990).

Then it was discovered that the area was very sensitive to the place where the $F_{M}$ was set (Bell and Hipkins 1985). Particularly, in cases where part of the PSII RCs remained uninhibited, it took a long time before $F_{M}$ was reached. As a consequence, the area grew strongly. In the pre-LED period, when shutters with opening times of $0.8-2$ ms were used, measurements in the presence of inhibitors like DCMU had to be made at very low light intensities to record as much as possible of the fluorescence transient. With modern shutterless LED-based systems, such measurements at light intensities of $3000 \mu \mathrm{mol}$ photons $\mathrm{m}^{-2} \mathrm{~s}^{-1}$ or more can be made without any problem. Such measurements have much better defined $F_{M}$ levels, which strongly reduces the problem with inhibited PSII RCs.

Using high light intensities, area ratios of alpha/beta/ gamma $=0.58: 0.33: 0.06$ were obtained for pea leaves (Tóth and Strasser 2005). With a simulation approach, Lazár et al. (2001) obtained similar values for wheat leaves: 0.64:032:0.04. The percentage of gamma centers agrees well with the percentage of $Q_{B}$ non-reducing centers determined using another method (Schansker and Strasser 2005). Based on present knowledge, the area growth of alpha centers should parallel the reduction of $Q_{A}$.

It should be noted that Trissl and Lavergne (1995) argued that changing rate constants during induction would preclude the use of the area as a measure for $Q_{A}^{-}$. However, if we assume that almost each charge separation reduces $Q_{A}$ (quantum yield of approximately 0.88 , see Question 6), then the area growth simply follows the gradual reduction of $Q_{A}$ in all PSII RCs and does not depend on different reactions and changing rate constants in individual RCs.

The area approach has also been applied to OJIP transients, for example, in the JIP test (Strasser and Strasser
1995; Strasser et al. 2004), but also by Joliot and Joliot (2002) and Tóth et al. (2007b). Joliot and Joliot (2002) showed that the regeneration of the area above the OJIP transient following a saturating pulse of light is defined by two exponential phases that they ascribed to the reoxidation of the acceptor side of PSI (fast phase) and the reoxidation of the PQ pool (slow phase). Tóth et al. (2007b) manipulated the redox state of the electron transport chain using anaerobiosis and showed that both the area between $F_{M}$ and the OJ rise (0-3 ms) and the area between $F_{M}$ and the JI rise $(3-30 \mathrm{~ms})$ linearly correlate with the $F_{J}$ intensity.

The area above the OJIP transient is dominated by the area above the JIP rise. Schansker et al. (2011) have provided evidence that the character of the JIP rise differs from that of the OJ rise. During the JIP rise the fluorescence yield per reduced molecule of $Q_{A}$ reduced increases. In this respect, the calibration of the area above the OJIP transient by the area between the J step and the OJ rise, as done in the JIP test, may not be the best approach. Instead, it is better to use the area between the OJ rise and $F_{M}$, taking $J$ at $3 \mathrm{~ms}$. In this way, the fluorescence yield increase is taken care of. This area would equate approximately 3 electrons, i.e., the reduction of the acceptor side of PSII. The JIP test approach yields for dark-adapted, nonstressed leaves a value $N$ of about 30 electrons (=total Area divided by the area between the OJ rise and the $\mathrm{J}$ step). Joliot and Joliot (2002) observed that PQ pool and PSI acceptor-side pool size were similar. For the JIP test approach this means 7-8 PQ molecules per PSII, which agrees quite well with a calculation of Lavergne et al. (1992). Kirchhoff et al. (2000), on the other hand, concluded that in stacked thylakoid membranes there are about 3-4 PQ molecules per PSII, which is a considerably lower value. The approach of Tóth et al. (2007b), using the area between OJ rise and $F_{M}$, yields for Phaseolus vulgaris leaves a value $N$ of approximately 31 and about 7 PQ molecules. Although the calculation in both cases is quite different, the outcome is quite similar.

The conformational change concept gives a rationalization for the relationship between area and electron transport chain redox state (Schansker et al. 2011, 2014). The induction of the conformational changes depends on the time interval $Q_{A}^{-}$is reduced before being reoxidized, and this in turn depends on the redox state of the electron transport chain.

The use of the Area-if normalized-is an effective way to detect changes in the redox state of the electron transport chain or changes in the stoichiometry PSII/PQ pool/PSI acceptor side of unstressed leaves. For stressed leaves, the Area can only be used if it has been ascertained that several criteria are met: The $F_{M}$ should be reached and the rate constants of different electron transport reactions should 
not be affected (too much). In the case of high-temperature stress, individual PSII RCs are knocked out. The remaining PSII reaction centers have to reduce more electron acceptors before $F_{M}$ is reached. In this case, the increase in the Area should be a function of the extent of inhibition of the PSII RCs as long as $F_{M}$ is still reached, which in severe cases is no longer true (Tóth et al. 2005a; see transients in Srivastava et al. 1997).

In summary, the Area is a useful tool to probe electron transport chain capacity.

A related parameter is $t_{F_{m}}$, the time needed to reach $F_{M}$. A physiological characterization of this parameter is still missing. It seems likely that this parameter has a strong sensitivity to the PSII/PSI ratio and the size of the PSI acceptor-side pool.

\section{Question 17: Can the 77 K fluorescence emission bands be assigned to specific photosynthetic complexes or processes?}

The interpretation of $77 \mathrm{~K}$ fluorescence emission spectra of cyanobacteria, algal cells and higher plants discussed in the previous paper (Kalaji et al. 2014a) was based on the analysis of fluorescence emission spectra measured on isolated complexes of, e.g., CP47 and CP43. The assignment of especially the bands associated with PSII has changed considerably in the last 10 years. In Fig. 5 an example of a $77 \mathrm{~K}$ spectrum (of Arum italicum thylakoid membranes) with the deconvoluted bands indicated is given.

The commonly accepted assignment of F695 to CP47 and F685 to CP43, as mentioned above, is based on the

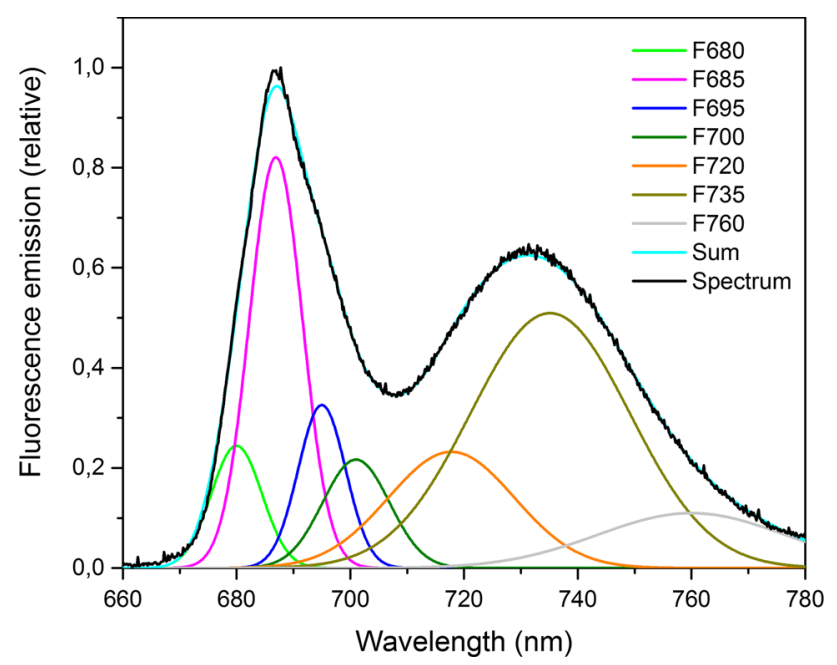

Fig. $577 \mathrm{~K}$ spectrum measured on Arum italicum thylakoid membranes with its deconvoluted bands indicated. For the assignment of the bands see the text of Question 17 (Pancaldi and Ferroni, unpublished data) analysis of the spectral properties of isolated complexes (Nakatani et al. 1984; van Dorssen et al. 1987; Alfonso et al. 1994; Groot et al. 1999).

Andrizhiyevskaya et al. (2005), studying the temperature dependence (4-293 K) of the fluorescence emission spectra, confirmed that F695 is emitted by CP47. The complex contains a $\mathrm{Chl}$ that absorbs light at $690 \mathrm{~nm}$ and excitation energy trapped by this Chl can only be transferred to the RC by uphill energy transfer. At low temperatures $(77 \mathrm{~K}$ or lower) this extra energy cannot be drawn from the environment and the excitation energy is irreversibly trapped giving rise to F695. Chls absorbing at $683 \mathrm{~nm}$ are found not only in CP43 but also in CP47. Also for excitation energy trapped on these Chls an uphill transfer to the RC is needed, but the energy difference is smaller. Lowering the temperature only leads to a slowdown of energy transfer. Andrizhiyevskaya et al. (2005) further observed the F685 band in CP47-RC complexes, but its intensity was smaller than in core complexes that also contain CP43. The authors concluded that F685 should be assigned to specific Chls in both CP47 and CP43.

The subunit approach came under criticism by a study of Krausz et al. (2005) who compared the emission of PSII holocomplexes with those of isolated LHCII, CP43, CP47 and a minimal PSII core unit formed by D1/D2/cyt $b_{559}$ and concluded that a combination of subunit spectra does not add up to the emission spectrum of intact PSII. Like Andrizhiyevskaya et al. (2005), the authors suggested that the PSII fluorescence emission spectrum depends on energy transfer bottlenecks due to a few $\mathrm{Chl}$ molecules in CP43 (ca. 2.5 Chls) and CP47 (ca. 1 Chl).

In the majority of the earlier studies a significant emission contribution from PSII RCs was excluded. Chen et al. (2015) made an extensive comparison between published results and new fluorescence emission spectra recorded on isolated PSII cores of spinach and from redissolved PSII core crystals of the cyanobacterium Thermosynechococcus elongatus. The authors showed that isolation procedures that preserve the intactness of the PSII core result in nearly overlapping $77 \mathrm{~K}$ emission spectra of cyanobacteria, the green alga Chlamydomonas reinhardtii and spinach. Four main emission sources governing the spectral outline in the PSII region were identified: (1) F695, originating from intact CP47; (2) two emission bands from destabilized CP47, peaking at $691 \mathrm{~nm}$ (FT1, matching that of isolated CP47 found in previous studies by van Dorssen et al. 1987) and at $685 \mathrm{~nm}$ (FT2); (3) emission near 686-687 nm, originating from pigments of the PSII reaction center. It was also concluded that CP43, whose emission peak is actually at $683.3 \mathrm{~nm}$ and not at 685-686 nm (Dang et al. 2008; Reppert et al. 2010), is not an important emission source of F685 in intact PSII. The authors also refer to the observation of Sun et al. (2015) 
that interaction of LHCII with purified PSII core increases F685 considerably, which would further support their assignment of F685 to PSII RC pigments. In other words, in intact PSII core, the main source for F685 is proposed to be the PSII reaction center.

D'Haene et al. 2015 came with an alternative interpretation of F695. In PSII mutants of the cyanobacterium Synechocystis lacking PsbH, a small PSII subunit, F695 is not detected. The authors suggest that the interaction of $\mathrm{PsbH}$ with a single monomeric Chl ligated to His114 of CP47 is responsible for F695.

The story of the PSII $77 \mathrm{~K}$ emission band assignment is a good illustration of the potential problems of using isolated subunits to study processes affecting the whole complex. In this case, the whole is more than the sum of the parts.

The assignment of the fluorescence emission associated with PSI at around $720 \mathrm{~nm}$ is more straightforward. The major fluorescence emission band of PSI is remarkably variable among different species (Murata et al. 1966b) with peak emission occurring between 710 and $730 \mathrm{~nm}$. Isolation of subunits from PSI particles allowed a more detailed characterization of PSI emission (Mullet et al. 1980; Argyroudi-Akoyunoglou 1984; Kuang et al.1984). In higher plants two main emission sources were identified at $77 \mathrm{~K}$ : F720 and F735 (Mukerji and Sauer 1990; Pålsson et al. 1995). In the green alga Chlamydomonas reinhardtii, LHCIPSI emission is quite blue-shifted compared to higher plants, i.e., a peak at ca. $715 \mathrm{~nm}$ instead of $735 \mathrm{~nm}$ (Garnier et al. 1986; Ferroni et al. 2011). Croce et al. (1997) showed that actually three Chl spectral forms exist in native PSI from maize thylakoids, having emission maxima at 720, 730 and $742 \mathrm{~nm}$. Analyzing barley mutants lacking individual LHCI subunits, Knoetzel et al. (1998) linked the Lhca1 and Lhca4 subunits of LHCI to emissions at 732 and $742 \mathrm{~nm}$, respectively, but only when such LHCI antennae were associated with PSI. A simplified band attribution can, therefore, be proposed: PSI core emits F720, but is also characterized by a short wavelength emission at $690 \mathrm{~nm}$ (Croce et al. 1997). Assembly of LHCI-PSI complexes gives rise to longer wavelength emissions with increased intensity, collectively giving rise to F735. In cyanobacteria, where PSI typically is organized in trimers instead of monomers, a further redshift in emission gives rise to F760 (Shubin et al. 1992; Karapetyan et al. 2014). Origin of PSI fluorescence bands is attributed to the "red chlorophylls" bound to PSI core, Lhca1/4 and Lhca2/3; the emission sources are probably dimers, trimers or aggregates of Chls which absorb light at wavelengths longer than $700 \mathrm{~nm}$, necessitating uphill energy transfer at $77 \mathrm{~K}$ as described above for PSII (reviewed by van Grondelle and Gobets 2004).

An important contribution to the $77 \mathrm{~K}$ spectra comes from LHCII. The presence of LHCII, formed by trimers of
Lhcb1-3 subunits in higher plants, gives the photosystems a high degree of flexibility, which is still the subject of intense study. The following interactions have been observed so far: (1) LHCII association with PSII core dimers to form LHCII-PSII supercomplexes (Boekema et al. 1999); (2) LHCII association with PSI in the so-called LHCII-PSI state transition complex to balance excitation between PSI and PSII (Pesaresi et al. 2009; Wientjes et al. 2013b); (3) aggregation of LHCII trimers related to the transition from the harvesting to the quenched state $\left(q_{\mathrm{E}}\right)$ (Johnson et al. 2011); (4) monomerization of LHCII trimers upon long-term acclimation to high light to reduce energy transfer to photosystems (Bielczynski et al. 2016); (5) LHCII trimers surrounding PSI-PSII megacomplexes (Yokono et al. 2015) and/or forming a bridge between PSI and PSII in such megacomplexes (Grieco et al. 2015; Suorsa et al. 2015) mediating photoprotective energy spillover from PSII to PSI (Yokono et al. 2015; Ferroni et al. 2016). Because LHCII can serve PSII and/or PSI, the relative size of F685 and F720 is dependent on the preillumination conditions (see also Question 8).

The F680 emission of LHCII, as demonstrated in isolated LHCII monomers and trimers (Kirchhoff et al. 2003; Caffarri et al. 2004; Karapetyan 2004), is not usually found as a distinct peak in $77 \mathrm{~K}$ spectra of thylakoids, reflecting the very efficient excitation energy transfer from LHCII to PSII in LHCII-PSII supercomplexes. Association of LHCII with PSII core increased the intensity of F695 (greening pea plants, Srivastava et al. 1999) and/or F685 (in vitro incorporation of LHCII in PSII cores, Sun et al. 2015), testifying to an increased efficiency of exciton transfer to the reaction centers. Conversely, release of LHCII trimers enhances F680. There are several lines of evidence linking F680 to free LHCII, among which LHCII release from grana cores induced by thylakoid unstacking (van der Weij-de Wit et al. 2007), LHCII release from grana margins induced by digitonin treatment of isolated thylakoids (Grieco et al. 2015), and also steady-state conditions in which LHCII trimers are overly abundant as compared to photosystem cores (Pantaleoni et al. 2009; Ferroni et al. 2013). Native LHCII trimers have a strong tendency to aggregate in vitro, leading to the appearance of a new longwavelength band, F700, also accompanied by a quenching of fluorescence, which could support a role of LHCII aggregates in excess energy dissipation (Mullet et al. 1980; Ruban and Horton 1992; Karapetyan 2004; Schaller et al. 2011). F700 has been recorded also in vivo in intact leaves (Šiffel and Braunová 1999; Oh et al. 2003). The LHCII aggregates that originated in leaves upon a $\mathrm{CO}_{2}$ deficit yielded a F700 band with high fluorescence yield (Šiffel and Braunová 1999). It is likely that, in vivo, different populations of LHCII aggregates can be formed, either engaged in thermal dissipation or not. 
In summary, much progress has been made with respect to the assignment of fluorescence emission bands to particular subunits at $77 \mathrm{~K}$. For the applied user, this assignment is important for the interpretation of changes in the emission spectra, especially in the case of PSII mutants or environmental stress effects. The molecular mechanisms behind the emission bands represent key information for the simulation of these bands (e.g., Reppert et al. 2010; Renger and Schlodder 2011).

\section{Question 18: What is the relationship between delayed and prompt fluorescence?}

Prompt fluorescence (PF) is due to the emission of light following absorption of a photon caused by the return of an excited Chl molecule to the ground state, which takes only 1.5-1.8 ns (Brody and Rabinowitch 1957; Barber et al. 1989; Krause and Weis 1991). Strehler and Arnold (1951) discovered that there is a second type of fluorescence emission that can be detected for quite long times after switching off the light. This type of fluorescence is related to recombination reactions, which, depending on the recombining charge pair, occur with a lifetime of $\sim 40 \mu \mathrm{s}$ for $\mathrm{P} 680^{+} / Q_{A}^{-}$(Govindjee and Jursinic 1979) or tens of seconds in the case of the recombination between $S 2$ or $S 3$ and $Q_{B}^{-}$(e.g., Rutherford et al. 1984). Half-times for the recombination between $Q_{A}^{-}$and $\mathrm{P}^{-} 60^{+}$determined for samples with a destroyed donor side (Tris-washed) give a half-time of about 120-130 $\mu$ s (Conjeaud and Mathis 1980; de Wijn and van Gorkom 2002). This type of fluorescence emission is called delayed fluorescence (DF) (e.g., Wraight and Crofts 1971; Goltsev et al. 2009) or delayed light emission (DLE) (e.g., Arnold and Thompson 1956; Srivastava et al. 1999) and is much weaker (about 100-fold) than PF (Jursinic and Govindjee 1982; Arnold 1991). In summary, the lifetime of different DF components is determined by the lifetime of the corresponding PSII state (charge pair). The emission spectrum of prompt and delayed fluorescence is identical (Strehler and Arnold 1951; Arnold and Thompson 1956; Sonneveld et al. 1980; Grabolle and Dau 2005). As described by the reversible radical pair model of Schatz et al. (1988) recombination can lead to the formation of "secondary excited" $\mathrm{Chl}$ *. This excitation energy can be transferred back to the antenna. There it is emitted by the same mechanism as PF (e.g., Grabolle and Dau 2005). Like PF, DF is predominantly a PSII phenomenon and it always involves a recombination between a negative charge on the acceptor side of PSII and a positive charge on the donor side of PSII. Figure 6 shows a schematic representation of the conditions under which PF and DF emission occurs. Details on the characteristics of DF can be found in a number of reviews (Lavorel 1975; Amesz and van Gorkom 1978;

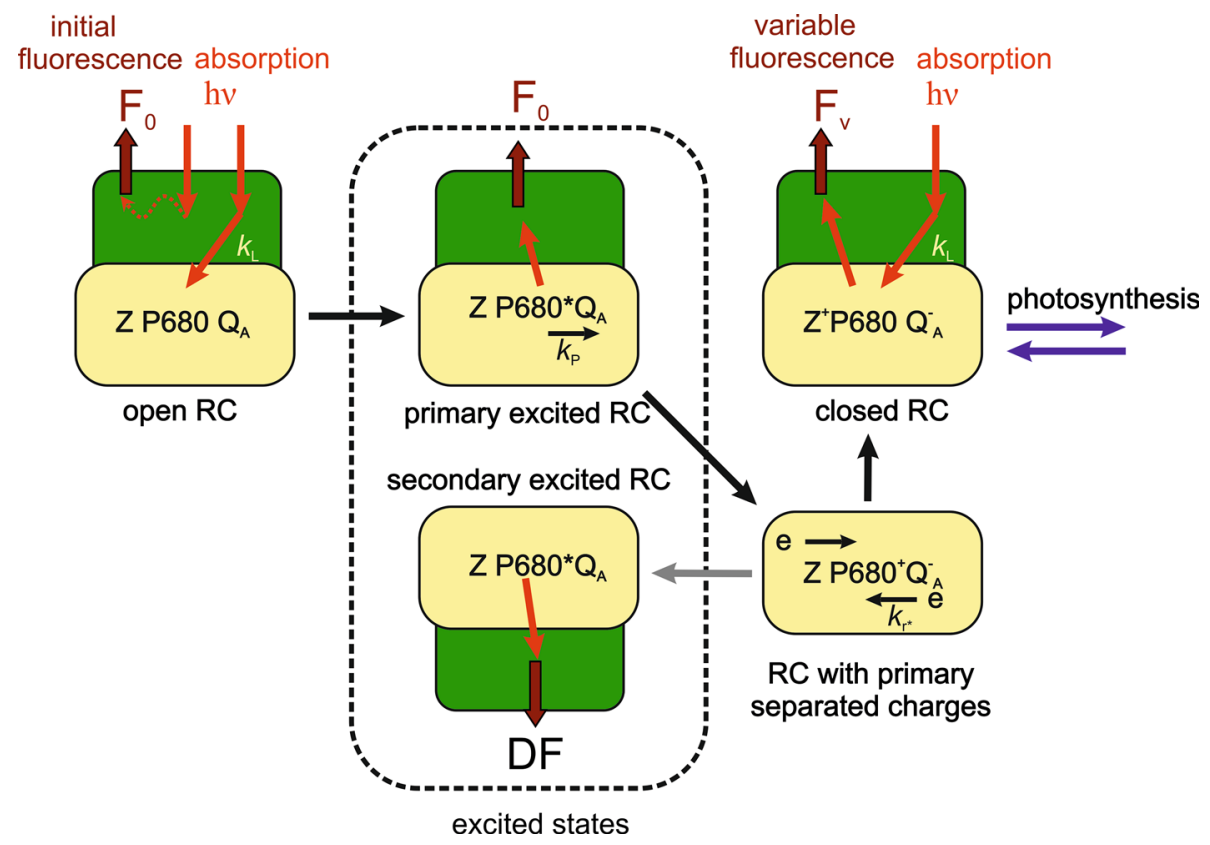

Fig. 6 Illustration of the difference between $F_{O}$ and DF. Following absorption of a photon the excitation energy may be lost in the antenna and re-emitted as fluorescence $\left(F_{O}\right)$. The excitation energy may induce a charge separation, which may be followed by electron transfer to $Q_{A}$ or a recombination reaction. In the latter case the energy may again be lost as fluorescence $\left(F_{O}\right)$. Emission occurs at subnanosecond times following excitation. Fluorescence emission induced by recombination between $Q_{A}^{-}$and the PSII donor side leads to a delay in the emission time with the fluorescence emission occurring $\sim 40 \mu \mathrm{s}$ or longer following excitation and is called delayed fluorescence (DF) (Goltsev, unpublished data) 
Malkin 1979; Lavorel et al. 1982; Jursinic 1986; Veselovskii and Veselova 1990; Gaevsky and Morgun 1993; Radenovic et al. 1994; Tyystjärvi and Vass 2004; Goltsev et al. 2009; Kalaji et al. 2012a). The older reviews predate the introduction of the reversible radical pair mechanism and contain extensive discussions on the mechanism of DF. These articles are good sources for experimental DF phenomena though.

\section{Question 19: What is the relationship between delayed fluorescence (DF) and thermoluminescence (TL)?}

As noted above, each charge pair has its own particular recombination time. Based on their specific lifetimes, the different charge pairs can be identified and their population/amplitude and recombination rate characterized (reviewed by, e.g., Vass and Govindjee 1996; Vass 2003). Thermoluminescence (TL), light emission as a result of temperature-dependent recombination of charge pairs, is a special application of DF, although it has to be noted that not all DF phenomena have a TL counterpart (Vass and Govindjee 1996). In addition, there are high-temperature TL bands that are due to oxidative chemiluminescence $\left(50-70{ }^{\circ} \mathrm{C}\right)$ (Hideg and Vass 1993; Skotnica et al. 1999) and lipid peroxidation $\left(130-140{ }^{\circ} \mathrm{C}\right.$ ) (Ducruet and Vavilin 1999). As the temperature is lowered, charge recombinations become gradually inhibited. Below approximately $-40{ }^{\circ} \mathrm{C} /-45^{\circ} \mathrm{C}$ no charge recombinations occur anymore and induced charge pairs can be stabilized (Brudvig et al. 1983). For TL, there are different illumination strategies. It is possible to give one or more single turnover flashes at, for example, $-20{ }^{\circ} \mathrm{C}$, at which temperature it is still possible to induce stable charge separations in practically all reaction centers, followed by a rapid lowering of the temperature to stabilize the induced state. Alternatively, it is possible to illuminate a sample for a much longer time in the range -60 to $-80{ }^{\circ} \mathrm{C}$ to induce a single charge separation in all reaction centers (Brudvig et al. 1983). This does not induce $\mathrm{Chl}$ or Car radicals as suggested by Stirbet and Govindjee (2012); to induce these radicals the temperature has to be lowered further (e.g., Faller et al. 2001). Inversely, upon warming the sample, the different charge pairs can gradually recombine; each charge pair with its own typical peak temperature (Vass 2003). Longer recombination times go together with higher TL peak temperatures. The $Q$ band, the charge recombination between $Q_{A}^{-}$and the $S 2$ or $S 3$ state of the OEC, peaks around $0{ }^{\circ} \mathrm{C}$, whereas the B band, the charge recombination between $Q_{B}^{-}$and the $S 2$ or $S 3$ state of the OEC, peaks at considerably higher temperatures. TL measurements become distorted during the transition from the frozen to the thawed state (Vass et al. 1981). To minimize the effects of this transition, different solutions have been found, for example a stronger heating around $0{ }^{\circ} \mathrm{C}$ (Ducruet and Miranda 1992) or the presence of 30 or $60 \%$ glycerol (Demeter et al. 1985). See Ducruet and Vass (2009) for a practical guide to the application of TL.

TL measurements are destructive. A sample does not survive the freezing/heating cycle that is applied during a measurement. DF measurements, on the other hand, are non-destructive. Even so, TL measurements have been more popular than DF measurements due to the absence of commercially available instruments for the measurement of DF. Such an instrument is now available on the market (MPEA, Hansatech Instruments Ltd, UK; see Question 21). It may also be mentioned that the analysis of TL measurements is better developed (DeVault et al. 1983; Vass 2003) than the analysis of DF transients. TL measurements have been used extensively for the characterization of PSII mutants (e.g., mutants that affect charge recombination properties) (e.g., Etienne et al. 1990; Cser and Vass 2007) and treatments that change the state of PSII (e.g., $\mathrm{Cl}^{-}$and $\mathrm{Ca}^{2+}$ depletion from the donor side of PSII or the effects of the presence of herbicides bound to the $Q_{B}$ site of PSII) (e.g., Krieger et al. 1993; Demeter et al. 1993; Bock et al. 2001). It is also a convenient technique for the study of environmental stresses that affect PSII (e.g., Mohanty et al. 1989; Hideg et al. 1993; Tóth et al. 2005a).

\section{Question 20: Does a recombination reaction always lead to DF emission?}

PF intensity is linearly related to the actinic light intensity (see, e.g., Schansker et al. 2006). In the case of DF only absorbed light that induces a stable charge separation can lead to DF and especially at high light intensities, this is only a small fraction of the absorbed light. In addition, DF emission is much more spread out in time because the DFinducing charge recombinations can be due to different charge pairs, with different recombination times. A third factor that reduces the DF yield is the existence of several recombination pathways of which only one leads to DF (see, e.g., Krieger-Liszkay and Rutherford 1998 and Cser and Vass 2007 for a discussion of this point). Cser and Vass (2007) give an overview of the different pathways: (1) a direct recombination between $Q_{A}^{-}$and $\mathrm{P}_{680^{+}}$via tunneling, which is non-radiative; (2) recombination via the triplet state of $\mathrm{P} 680^{+} \mathrm{Pheo}^{-}$, which is also non-radiative, but there is an increased probability of singlet oxygen formation (see Question 13); (3) recombination via the singlet state of $\mathrm{P} 60^{+} \mathrm{Pheo}^{-}$, which decays via light emission. Pathways 2 and 3 are called indirect charge recombination pathways. Krieger-Liszkay and Rutherford 
(1998) showed that DCMU bound to the $Q_{B}$ site increases the midpoint potential of $Q_{A}$, stabilizes the charge separation (shift of TL bands to higher temperatures) and increases the probability that recombination occurs via tunneling (pathway 1). Bromoxynil, on the other hand, decreases the midpoint potential of $Q_{A}$, destabilizes the charge separation (shift TL bands to lower temperature) and increases the probability that recombination occurs via pathways 2 and 3). Cser and Vass (2007) confirmed this interpretation using mutants in which amino acids near Pheo and P680 were modified using site-directed mutagenesis. The authors showed that the free energy difference between $\mathrm{P} 680$ in the excited state and $\mathrm{P} 680^{+} \mathrm{Pheo}^{-}$is an important determinant of TL emission intensity.

\section{Question 21: How can PF and DF be simultaneously measured?}

PSII reaction centers and antennae emit about 100 times more PF than DF. In addition, the emission spectra of both types of fluorescence emission are identical (Strehler and Arnold 1951; Arnold and Thompson 1956; Sonneveld et al. 1980; Grabolle and Dau 2005) (see Question 18). As a consequence, both cannot be detected at the same time and thus a separation strategy is needed. Two approaches for the simultaneous monitoring of PF and DF signals have been developed: (1) quasi-continuous illumination of darkadapted samples and (2) illumination of a dark-adapted sample by single pulse (usually by a laser) [see, e.g., Steffen et al. (2001) for such a experimental setup and Belyaeva et al. (2015) for a discussion of laser-flash-induced fluorescence data], or by continuous illumination. The results of these two methods differ.

The first approach has been used for the evaluation of the induction kinetics of the two fluorescence signals over short time periods (seconds) (Wraight and Crofts 1971; Zaharieva and Goltsev 2003; Strasser et al. 2010), whereas the second approach has been used for both the analysis of induction kinetics (e.g., Schansker et al. 2011) and the analysis of PF and DF relaxation kinetics within wider time intervals (minutes to hours) (Katsumata et al. 2008, Berden-Zrimec et al. 2011).

The first approach has been successfully implemented using the Multifunction Plant Efficiency Analyzer (MPEA, Hansatech Instruments Ltd, UK). The sample is illuminated by a series of red light pulses of variable duration (produced by light-emitting diodes), followed by short dark periods (duration: 1/3 of the light pulses). This type of illumination is often called quasi-continuous. Prompt fluorescence is measured during the illumination periods, and the decaying DF signal is recorded during the dark intervals. The maximum rate of digitization of both signals in the M-PEA is 1 point per $10 \mu \mathrm{s}$. It should be noted that the dark intervals reduce the effective light intensity by onethird. Thus, $5000 \mu \mathrm{mol}$ photons $\mathrm{m}^{-2} \mathrm{~s}^{-1}$ light pulses yield an effective light intensity of $3334 \mu \mathrm{mol}$ photons $\mathrm{m}^{-2} \mathrm{~s}^{-1}$. In addition, during the dark intervals the dark reactions continue (electron flow), whereas the light reactions (charge separations) stop, changing the relationship between dark and light reactions.

Using quasi-continuous illumination, DF induction transients complementing the PF induction transients can be constructed. To do this, the DF intensities measured during a particular dark interval for all dark periods are selected and averaged, and then used as single points for the DF induction curve. By selecting different decay intervals, induction curves can be constructed that show DF kinetic components with different lifetimes (Kalaji et al. 2012a; Fig. 7a).

Using the second approach, $\mathrm{Chl} a$ fluorescence decay is recorded after a short (ns) laser pulse or after continuous illumination. The strong fluorescence signal registered
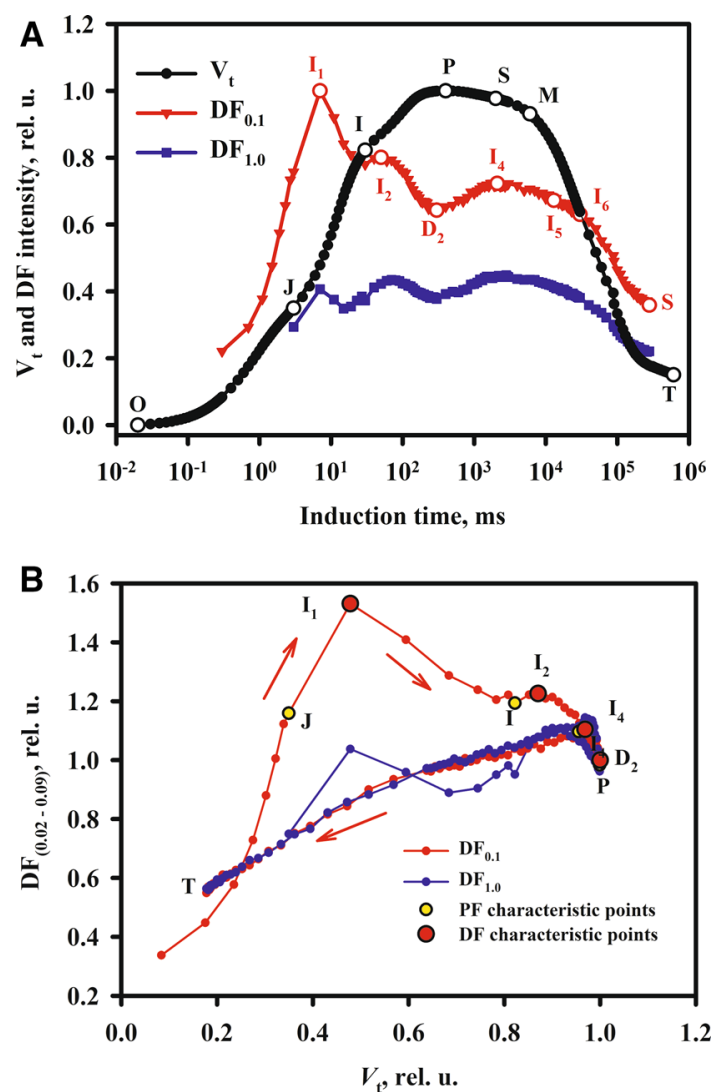

Fig. 7 Kinetics of prompt fluorescence (PF) induced by a pulse of $5000 \mu \mathrm{mol}$ photons $\mathrm{m}^{-2} \mathrm{~s}^{-1}$, interspaced by short intervals of darkness to measure the delayed fluorescence (DF) kinetics. a PF intensity, DF intensity following $0.1 \mathrm{~ms}$ of darkness and the DF intensity following $1 \mathrm{~ms}$ of darkness as a function the time of illumination; b DF intensity following 0.1 and $1 \mathrm{~ms}$ of darkness as a function of the PF intensity (Goltsev, unpublished data) 
during the first few nanoseconds following illumination is due to PF, while at longer times after illumination the emitted light is the result of charge recombinations (i.e., DF) (Goltsev et al. 2009). Alternatively, single light pulses of variable length (e.g., 1-200 ms) can be given, during which PF is measured, followed by a dark period during which DF is measured (Schansker et al. 2011). The M-PEA also allows this type of measurement. This approach is much more time-consuming than method (1), because each time point is a separate measurement, but has the advantage that the intensity of the actinic light is also the effective light intensity and, therefore, the results can be related to OJIP transients on a one-to-one basis.

\section{Question 22: How to compare PF induction transients with induction curves of different DF decay components?}

The DF induction curve is often compared to the simultaneously measured PF induction curve. To allow a direct comparison of the maxima for both fluorescence types, they are superimposed on each other on the same timescale (Govindjee and Papageorgiou 1971; Krause and Weis 1991; Malkin et al. 1994; Goltsev et al. 2003, 2005, 2009; Strasser et al. 2010; Kalaji et al. 2012a; Fig. 7a). For the construction of DF induction transients, see Question 21.

During induction, different reactions occur in parallel in different RCs. Upon turning off the light, different reoxidation and recombination reactions all occur separately with their own particular rate constants (for PF see, e.g., Schansker et al. 2005). Since PF is measured during induction (a mix), and DF during dark intervals (in time separated reactions), comparing $\mathrm{PF}$ and $\mathrm{DF}$ is not straightforward (Mar et al. 1975) and needs an analysis framework.

To understand DF measurements during the first few ms of induction, it is important to understand what happens on the donor side of PSII. The different $\mathrm{S}$ states each transfer an electron to $\mathrm{Tyr}_{Z}^{+}$with different transfer times: $S 0 \rightarrow S 1=30 \mu \mathrm{s}, S 1 \rightarrow S 2=100 \mu \mathrm{s}, S 2 \rightarrow S 3=300 \mu \mathrm{s}$, $S 3 / 4 \rightarrow S 0=1.5 \mathrm{~ms}$ (see Grabolle and Dau 2007 and references therein). This sequence shows that the lifetime of the couple $\left(\mathrm{Tyr}_{\mathrm{Z}} / \mathrm{P} 680\right)^{+}$increases as a function of the $S$ state. As shown by Grabolle and Dau (2005) the fast $\mu$ s DF component increases on the first three single turnover flashes reaching a maximum intensity on going toward the $S 4$ state. These authors also showed for the ms DF component that only the state induced by three flashes (on going from $S 3$ via $S 4$ to $S 0$ ) still yields a high DF intensity. After a ms, $\mathrm{Tyr}_{\mathrm{Z}}^{+}$has been re-reduced by the oxygen-evolving complex going to the $S 1, S 2$ and $S 3$ states.
According to simulations (Lazár 2003) and actual DF measurements (Schansker et al. 2011, Kalaji et al. 2012a) the maximum DF intensity/maximum $\left[\mathrm{P} 680^{+}\right]$occurs after 3-4 ms (I1 peak) and coincides with the $J$ step or slightly after. It is likely that the $\mathrm{K}$ peak (in high-temperature treated samples observed after $\sim 300 \mu$ s of illumination) is equal to one charge separation and, therefore, the $S 2$ state (Tóth et al. 2007a). Simulations of Lazár (2003) suggest that the second charge separation peaks around $1 \mathrm{~ms}$ of illumination ( $S 3$ state). And as mentioned above, the peak DF intensity ( $S 4$ state) occurs after 3-4 ms. This reaction sequence accounts for the DF rise to the I1 peak.

Using single turnover flashes, several $\mathrm{S}$ state cycles can be detected (see Grabolle and Dau 2005 for DF). OJIP transients, on the other hand, are induced by much lower light intensities that are much less concentrated in time. As a consequence the $S$ states will dampen (due to double hits and misses; Kok et al. 1970) more quickly. Probably, already shortly after reaching the $\mathrm{J}$ step, the donor sides of the PSII RCs will become desynchronized. This means that, at all times, there are 25\% S0, 25\% S1, 25\% S2 and $25 \% S 3$ and all donor side effects will cancel each other out. Once this has happened the acceptor side of PSII starts to determine DF again. This is observed beyond the $\mathrm{J}$ step where an inverse relationship between DF and PF is observed (e.g., Schansker et al. 2011; Kalaji et al. 2012a). Between $J$ and $P$ the ETC becomes gradually reduced (Schansker et al. 2005). As the PQ pool becomes reduced the availability of oxidized PQ decreases. This means that it takes longer before an oxidized PQ molecule binds to the $Q_{B}$ site and it takes longer before $Q_{A}^{-}$can again become reoxidized. This has two effects: $P F$ increases and DF decreases because only following the re-oxidation of $Q_{A}^{-}$a new charge separation can occur, and only then there is again a transient induction of $\mathrm{P} 60^{+}$(see Schansker et al. 2011, 2014 and Oukarroum et al. 2012 for a more in-depth discussion of this point).

The position of the I1 peak is sensitive to the light intensity. At a relatively low light intensity (500 $\mu \mathrm{mol}$ photons $\mathrm{m}^{-2} \mathrm{~s}^{-1}$; i.e., an effective light intensity of $334 \mu \mathrm{mol}$ photons $\mathrm{m}^{-2} \mathrm{~s}^{-1}$ ), the initial DF induction is rather slow and $\mathrm{I} 1$ is reached after about $23 \mathrm{~ms}$ (Kalaji et al. 2012a, b). Destruction of the Mn cluster changes the DF response. In that case the lifetime of $\mathrm{P} 680^{+}$ increases and the $Q_{A}^{-}$concentration strongly decreases (see Oukarroum et al. 2012).

Two DF induction curves are shown in Fig. 7a. They represent different DF decay intervals: 20-90 $\mu \mathrm{s}$ and 0.1-0.99 ms. The characteristic points of the DF induction are indicated according to the nomenclature proposed by Goltsev and Yordanov (1997) and Goltsev et al. (2005, 2009). The maxima (denoted by $I$ ) and minima (denoted by $D$ ) are numbered according to their position on 
the DF induction curve (I1, I2, D2 up to I6; $S$ is steady-state DF level).

Similarly to the PF induction curve, the DF induction curve can be divided into fast and slow phases (Itoh et al. 1971; Itoh and Murata 1973; Malkin and Barber 1978; Goltsev and Yordanov 1997; Goltsev et al. 2003, 2005; Fig. 7). The fast phase lasts for about $300 \mathrm{~ms}$ and coincides with the OJIP transient of PF. The slow phase lasts several minutes, reaching a stationary level after 5-10 min of illumination by quasi-continuous actinic light. Two maxima I1 and I2 (sometimes with a minimum D1 in between) are observed in the fast phase, after which DF drops to a minimum labeled as D2 (Goltsev and Yordanov 1997; Goltsev et al. 2003; Zaharieva and Goltsev 2003; Kalaji et al. 2012a). The second maximum (I2) appears at about 60-100 ms during the IP phase of PF. I2 strongly depends on the actinic light intensity; at high light intensities it is visible only as a shoulder (Schansker et al. 2011; Kalaji et al. 2012a).

The correlation between simultaneously recorded PF and DF in one sample can be visualized in a "phase diagram" (Malkin et al. 1994; Goltsev et al. 2003; Schansker et al. 2011; Kalaji et al. 2012a). In a 2D graph, DF values collected from different dark delay intervals are plotted as a function of relative double-normalized PF, $\mathrm{V}_{t}$ (Fig. 7b). For the two plots there is a long induction interval, where the intensities of PF and DF change synchronously (inversely). This appears on the diagram as an almost linear section between phases $S$ and $T$ of the PF transient. During this time interval both PF and DF intensities are modified, and it has been suggested that this is mainly due to changes in fluorescence quantum yield (Lavorel 1975; Goltsev et al. 2003). In the fast phase of induction (OJIP), PF and DF deviate from linearity for the reasons discussed above. In the first part of the diagram the changes are most pronounced for DF recorded during the 20- to 90- $\mu$ s decay interval. The dependence between DF and PF is not linear but almost quadratic, which means that PF increases initially much faster than DF. In the time range between points $\mathrm{I} 1$ and D2, DF shows a negative correlation with $\mathrm{PF}$-micro- and millisecond DF decreases when PF increases from $J$ to $I$ and $P$. See above for a discussion of the reasons for this relationship.

Between 0.5 and $5 \mathrm{~s}$ DF and PF are again inversely proportional. DF increases from D2 to I4, initially, as a result of the activation of FNR, due to which electron flow restarts (Satoh 1981; Harbinson and Hedley 1993; Schansker et al. 2003, 2008), $Q_{A}$ starts to turnover again (is re-oxidized and then reduced again), which is accompanied by a transient $\mathrm{P} 680^{+}$generation and this leads to an increase of DF emission (and a decrease of PF). The restarted electron flow is also accompanied by a further energetization of the thylakoid membranes. External electric fields are known to stimulate recombination reactions (Vos et al. 1991; Dau and Sauer 1992), and the naturally induced electric field may, therefore, also have an effect on DF emission.

In summary, during induction PF follows the reduction of the electron transport chain, whereas the fast $40-\mu$ s and ms DF components are determined by the $\mathrm{P} 680^{+}$concentration of the population of PSII RCs, which is determined by the $S$ states and the turnover rate of $Q_{A}$. In other words, both signals monitor the reduction kinetics of the photosynthetic electron transport chain in different ways, providing complementary information.

\section{Question 23: How do the fluorescence parameters vary during the day?}

To produce comparable experimental data several factors are important. In the previous paper the variability among instruments and the extent to which parameters vary naturally was discussed (Kalaji et al. 2014a). With respect to the parameter statistics we want to refer to two additional studies (Lazár and Nauš 1998; Lazár et al. 2005). In the first study it is shown that the statistical distribution of eight OJIP-derived parameters does not follow the Gaussian distribution law, and in the second study it is shown that a stress like high-temperature stress affects the statistical distribution. Changes occurring in leaves during the day and their effect on leaf sampling form another factor. An important variable is the orientation of a leaf. Certain leaves are oriented toward the east and receive most of their daily light dose in the morning, whereas other leaves may be oriented toward the west and receive a large part of their daily light dose in the afternoon. Losciale et al. (2010) and Demmig-Adams et al. (2012) gave experimental examples of this. Another important factor is the angle of the leaf relative to the plane. Leaves that are oriented almost orthogonally will intercept only a relatively small part of the sunlight, whereas leaves oriented nearly parallel to the plane will intercept a large part of the sunlight. A steeper orientation allows a better distribution of the light over different layers of leaves (e.g., Ishida et al. 1999; Falster and Westoby 2003; Stewart et al. 2003). Another factor is wind. Leaves that move around in the wind will continuously change their orientation relative to the sun causing fluctuations in the intercepted light. These fluctuations are further modulated by the passage of clouds and sunflecks. The intensity of sunlight also varies over the day, being low in the early morning and late afternoon and peaking around noon. This natural pattern is responsible for the typical bell curves of parameters like $F_{V} / F_{M}$ or zeaxanthin + antheraxanthin content during the day (e.g., Demmig-Adams et al. 1996, 2012; García-Plazaola et al. 
1997; Bernacchi et al. 2006). In this sense, photosynthetic activity is strongly dependent on the perceived average light intensity, which ultimately drives long-term light acclimation. In shade leaves, having a lower photosynthetic capacity, saturation will be reached at lower light intensities than in sun leaves. It may be noted, on the other hand, that shade leaves exhibit a higher photosynthetic activity at low light intensities and a lower compensation point. Saturation is further modulated by temperature. With the exception of the light reactions (photophysical), photosynthetic electron transport, Calvin-Benson cycle and the photorespiratory pathway have a biochemical character and, as a consequence, photosynthetic activity is quite strongly temperature dependent. A lowering of the temperature will shift the light intensity at which saturation occurs to lower light intensities. Low temperatures associated with high light intensities cause severe winter photoinhibition in Mediterranean (Martínez-Ferri et al. 2004) and Alpine-Central European (Robakowski 2005) tree species. Another important variable is the humidity of the air. Dry air is likely to lead to high levels of evaporation, to which the plant may respond by (partially) closing its stomata (Lange et al. 1971; Schulze 1986). This lowers the uptake of $\mathrm{CO}_{2}$ and increases photorespiratory activity at the expense of the assimilatory activity (Medrano et al. 2002). Closing of the stomata will, however, increase the leaf temperature (e.g., Long et al. 2006). All these variables should be considered when designing a leaf sampling protocol for an experiment (Rousseau et al. 2013). Many researchers have experimentally characterized the diurnal pattern of Chl $a$ fluorescence parameters in C3, C4 and CAM plants (Adams and Demmig-Adams 1992; Franco et al. 1999; Pollet et al. 2009; Desotgiu et al. 2013). On bright days, solar radiation is supersaturating with respect to the photosynthetic capacity during a considerable part of the day in most plant species and regions of the world (Long and Humphries 1994). Midday high light conditions lead to a decrease in the maximum quantum yield under dark-adapted conditions $\left(F_{V} / F_{M}\right)$ and an increase in the rate constant for thermal dissipation reflected by higher levels of $q_{\mathrm{E}}$ (Gilmore et al. 1998; Adams III and Demmig-Adams 2004). Field observations show the progressive decrease in $F_{V} / F_{M}$, measured after $20-25$ min of dark adaptation, with the lowest values at midday (Desotgiu et al. 2013). An obvious candidate for this decrease is photoinhibition, which causes a loss/quenching of $F_{V}$ (Long and Humphries 1994). Normally, plants recover from photoinhibition during the night and $F_{V} / F_{M}$ reaches its maximum value before sunrise (predawn observations) (e.g., DemmigAdams et al. 2012). Dark acclimation with leaf clips for 20 min during daytime hours ensures the relaxation of the transthylakoid pH difference (Quick and Stitt 1989; Nilkens et al. 2010), inactivation of FNR (Schansker et al.
2006, 2008) and realignment of the chloroplasts within the cell (Cazzaniga et al. 2013; Kong and Wada 2014). Peter Jahn's zeaxanthin-dependent $q_{Z}$ quenching, on the other hand, needs considerably more time to recover (Nilkens et al. 2010; Jahns and Holzwarth 2012), just as the release of part of the PSII antenna proposed by Alfred Holzwarth (Holzwarth et al. 2009) and Roberto Bassi (Betterle et al. 2009), and even more time is needed to recover from photoinhibition (Horton and Hague 1988). These last three processes are all associated with a sustained $F_{V} / F_{M}$ reduction.

Photosynthesis frequently remains depressed during the afternoon hours (Correia et al. 1990; Pollastrini et al. 2013). Apart from photoinhibition and slowly reversing regulatory mechanisms, abscisic acid-induced stomatal closure in the afternoon (Tallman 2004) and feedback inhibition of photosynthesis by accumulating sucrose and/ or starch have been considered (see Paul and Foyer 2001 for a discussion). It is worth noting that nighttime recovery of photoinhibition may be inhibited or slowed down by low night temperatures (Strand and Lundmark 1987; Bussotti 2004). The temperature dependence of the repair cycle and its inhibition under low temperature conditions has been described under in vitro conditions (Aro et al. 1990).

The parameter $1-V_{J}$, which is an approximation of the relative amplitude of the thermal phase, follows the same diurnal pattern as the parameter $F_{V} / F_{M}$ (Desotgiu et al. 2013; Pollastrini et al. 2011, 2014). In the JIP test this parameter is associated with electron transport activity (Strasser et al. 2004); however, there are several processes to which this parameter is sensitive, including severe hightemperature stress associated with inactivation of the PSII donor side (Schreiber and Neubauer 1987; Srivastava et al. 1997; Tóth et al. 2007a), and a partially reduced PQ pool (Tóth et al. 2007b), but also certain regulatory mechanisms (Schreiber et al. 1995; Schansker et al. 2006). Photoinhibition, reducing the donation capacity of all PSII RCs together, may, in this respect, have the same effect on OJIP transient as high-temperature stress. The water status of plants may play an important role. Desotgiu et al. (2012a) observed for well-watered plants of Fagus sylvatica L. that $1-V_{J}$ increased at midday. The amplitude of the IP phase, which was shown experimentally to correlate with leaf PSI content in fully dark-adapted leaves, was always found to be enhanced during the noontime hours as well as in sun leaves (Cascio et al. 2010; Desotgiu et al. 2013; Pollastrini et al. 2014). However, the reason for this observation is different in these two cases. In dark-acclimated sun leaves the PSII/PSI ratio may be lower than in dark-acclimated shade leaves (Anderson et al. 1988) and this is expected to lead to an increased IP amplitude. If leaves suffer from a significant amount of photoinhibition at noontime, the electron donation capacity of PSII will be lower. This is 
comparable to illumination at a lower light intensity (cf. Schansker et al. 2011) or high-temperature stress (cf. Srivastava et al. 1997), both conditions that increase the relative amplitude of the IP phase. The electron transport rate (ETR), the effective quantum yield $\left(\Phi_{\text {PSII }}\right)$ and photochemical quenching $\left(q_{\mathrm{P}}\right)$ derived from PAM fluorimetry on light-adapted samples are higher during the day than at night (Larcher 2000; Pollet et al. 2009; Desotgiu et al. 2013). An explanation for this observation may be that leaves kept overnight in darkness may have lower sink activities and a reduced stomatal opening (Felle et al. 2000) compared to plants that have been exposed for hours to $\sim 600 \mu \mathrm{mol}$ photons $\mathrm{m}^{-2} \mathrm{~s}^{-1}$.

\section{Question 24: How do fluorescence parameters vary within a tree canopy?}

Leaves from a tree form a population, and each single leaf may differ from the others in terms of size, age and position in the tree canopy. The position of the leaf in the canopy (top vs. bottom leaves; outer vs. inner) determines primarily the exposure to sunlight and the differentiation between sun and shade leaves. Sun and shade leaves differ significantly in their photosynthetic apparatus and performance (Lichtenthaler et al. 1981; Anderson et al. 1988). Sun leaves have in general a lower Chl $a$ and $b$ content, smaller thylakoid stacks, lower LHCII content, higher Chl $a / b$ ratio, lower $\operatorname{Chl}(a+b) /$ carotenoid ratio and a higher PSI/PSII ratio when compared with shade leaves. Furthermore, sun leaves have a higher Calvin-Benson cycle capacity relative to the capacity of the electron transport chains, and more efficiently dissipate excess energy as heat, compared to shade leaves. Shade leaves, on the other hand, are more efficient at exploiting low PAR levels for photosynthesis, having a larger PSII antenna and more extensively stacked thylakoid membranes (Lichtenthaler et al. 1981).

The Chl $a$ fluorescence parameters most sensitive to sunlight exposure during the day are (1) the maximum quantum yield of primary photochemistry $\left(F_{V} / F_{M}\right)$ as a measure for the efficiency of the whole PSII population and the sensitivity of PSII to photoinhibition, and (2) nonphotochemical quenching (NPQ). NPQ induction in response to solar radiation is stronger in sun leaves than in shade leaves. Among the chlorophyll fluorescence parameters derived from OJIP transients, the amplitude of the IP phase, that reflects the relative PSI content (Oukarroum et al. 2009; Ceppi et al. 2012), is higher in sun leaves than in shade leaves, which is in agreement with the higher PSI/ PSII ratio.

The Chl $a$ fluorescence parameters are also sensitive to the age and/or senescence of leaves/needles in evergreen tree species and to the age of leaves appearing in the spring compared with those developed during the summer or autumn (Desotgiu et al. 2012b).

Some stress factors act preferentially on a specific side of the canopy. These factors include, e.g., wind blowing dominantly out of a particular direction and provoking desiccation of leaves, salt from the sea shore, and chemicals from local pollution sources. Other environmental factors, such as soil properties and air pollutants, affect the canopy in a different way. The effects of these factors depend on the physiology, ontogeny and the position of leaves in the crown. We can thus have damaged leaves at specific levels in the tree crown, e.g., ozone symptoms on leaves in the lower part of the crown, but not in the higher part (Desotgiu et al. 2012b).

Finally, when leaves are lost from the branches due to senescence, or damaged by biotic or abiotic factors, the photosynthetic activity of the remaining foliage may increase (Eyles et al. 2011). Such a response affects the value of several Chl $a$ fluorescence parameters, especially the amplitude of the IP phase of the OJIP transient (Desotgiu et al. 2012b).

\section{Question 25: What do Chl $a$ fluorescence measurements tell us about drought stress?}

The usefulness of individual Chl $a$ fluorescence parameters and protocols for evaluation of drought depends on the severity and duration of drought stress (Suresh et al. 2012). Mild-to-moderate drought stress causes a decrease in the photosynthetic rate, mainly due to stomatal closure, whereas drought has no direct effect on the capacity of individual metabolic reactions (Brestič et al. 1995; Cornic and Massacci 1996; Flexas and Medrano 2002). Recently, it was observed that blue-light-induced chloroplast movements are very sensitive to drought stress. Inhibition occurred already at RWC values considerably above $70 \%$, and was, therefore proposed to be a sensitive tool for small changes in RWC (Nauš et al. 2016). In typical C3 plants, critical leaf relative water content is about $70 \%$ and below this value non-stomatal effects occur. These phenomena are also reflected in $\mathrm{Chl} a$ fluorescence and calculated fluorescence parameters.

The most frequently used fluorescence parameter in photosynthetic or environmental research (including drought stress) is the maximum quantum yield of PSII photochemistry $\left(F_{V} / F_{M}\right)$. This parameter is easy to measure and is generally well accepted as a measure for the PSII status (or more correctly the status of the population of PSII RCs). However, it is highly insensitive to stomatal changes and other effects occurring under moderate drought stress (see Fig. 8, recorded during quick 


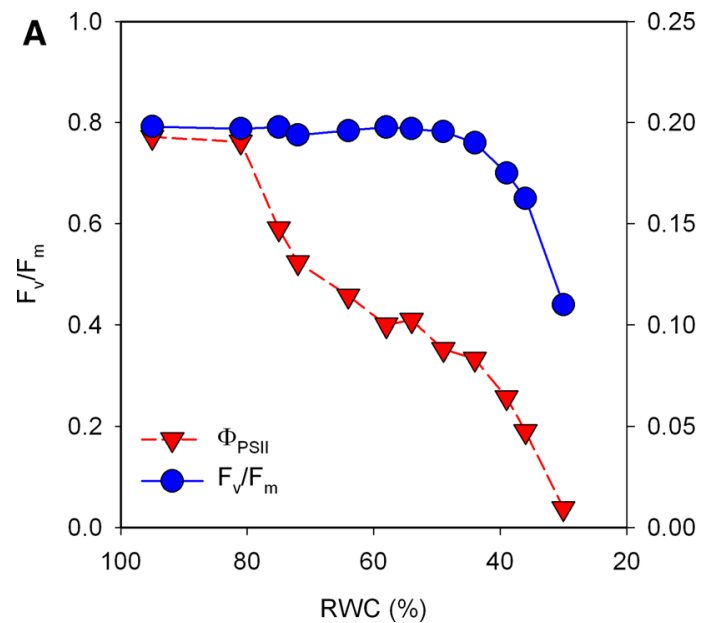

Fig. 8 Response of $\mathrm{Chl} a$ fluorescence parameters to drought stress. a The parameters $F_{V} / F_{M}$ and $\Phi_{\mathrm{PSII}}$ measured with a PAM fluorometer as a function of the relative water content (RWC); $\mathbf{b}$ the parameters

dehydration of a wheat leaf in very low light). $F_{V} / F_{M}$ values are extremely stable, starting to decline at a dehydration level that is lethal for typical leaves. If drought stress persists under field conditions for a longer period (days) the decrease in $F_{V} / F_{M}$ values can be dramatic (Živčák et al. 2008b). Hence, decreases in $F_{V} / F_{M}$ cannot be used to monitor early drought stress effects. However, the $F_{V} / F_{M}$ measurements during drought stress may draw attention to the effects of co-occurring stresses (high-temperature stress, photoinhibition, etc.) or to leaf senescence (Lu et al. 2002; Kotakis et al. 2014).

Both the slow and fast Chl $a$ fluorescence kinetics provide parameters sensitive to drought stress (Fracheboud and Leipner 2003; Oukarroum et al. 2007, 2009; Živčák et al. 2008a, 2014; Goltsev et al. 2012 ). The early decrease of the effective PSII quantum yield $\left(\Phi_{\mathrm{PSII}}\right)$ in droughtstressed leaves compared to well-hydrated ones is mainly due to lack of $\mathrm{CO}_{2}$ inside the leaf (closed stomata). In $\mathrm{C} 3$ leaves, the decrease of $\Phi_{\mathrm{PSII}}$ is not linearly correlated with net photosynthetic rate, as increased photorespiration efficiently consumes part of the electrons flowing through the photosynthetic electron transport chain. Moreover, alternative electron transport pathways also consume electrons generated by linear electron transport under drought stress conditions, complicating the physiological interpretation of the results (Živčák et al. 2013; Questions 11 and 13). For example, in $\mathrm{C} 3$ plants PSII activity $\left(\mathrm{O}_{2}\right.$ evolution rate) relative to Rubisco activity is $\sim$ equal to the carboxylase activity + the oxygenase activity which in turn correlates with PSII activity measured by Chl $a$ fluorescence analysis (Krall and Edwards 1992; Oberhuber et al. 1993).

In summary, measurements of slow fluorescence kinetics and calculation of quantum yields and electron transport rate (ETR) are useful for determination of drought stress

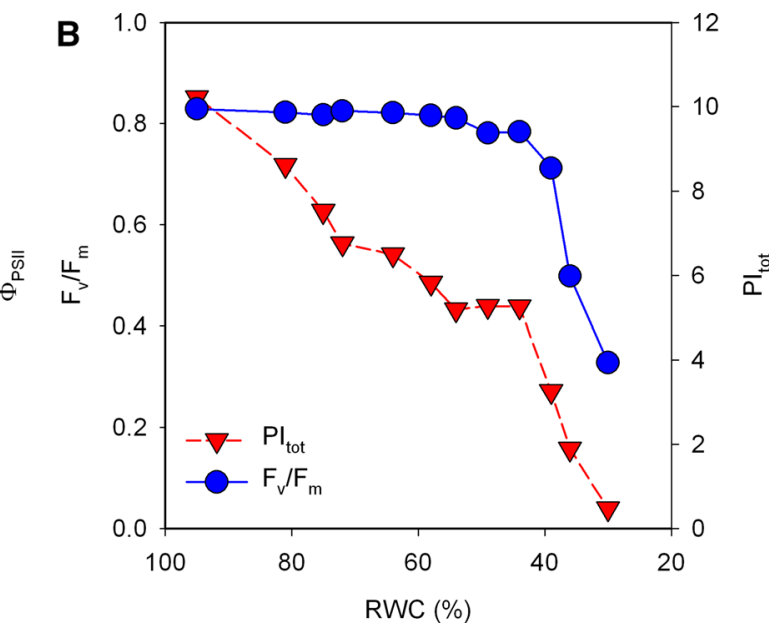

$F_{V} / F_{M}$ and $P I_{\text {tot }}$ measured with a HandyPEA as a function of the RWC (modified from Brestič and Živčák, Molecular Stress Physiology of Plants, Chapter 4, republished with permission of Springer)

effects, reflecting both stomatal and non-stomatal effects. However, such measurements during drought stress cannot be directly related to $\mathrm{CO}_{2}$ assimilation (Baker 2008). Indeed, the relative fluorescence decrease ratio $\left(R_{\mathrm{Fd}}\right)$ was shown to be more sensitive and better correlated with the photosynthetic $\mathrm{CO}_{2}$ assimilation rate than PSII quantum yield or ETR (Lichtenthaler et al. 2005a). $R_{\mathrm{Fd}}$ was introduced as a so-called vitality index, calculated as $R_{\mathrm{Fd}}=$ $\left(F_{P}-F_{S}\right) / F_{S}$, where $F_{P}$ and $F_{S}$ denote the fluorescence intensities at the fluorescence peak after $200-500 \mathrm{~ms}$ of illumination $\left(F_{P}\right)$, i.e., $P$ of the OJIP transient, and the fluorescence level in the steady state $\left(F_{S}\right)$ reached after a few minutes (usually 2-5 min) of illumination. When a plant is stressed, $F_{P}$ decreases due to processes such as photoinhibition and under stress conditions $F_{S}$ can also increase, leading to a decrease of $R_{\mathrm{Fd}}$ (Lichtenthaler et al. 1986). Lichtenthaler (2013) showed that $R_{\mathrm{Fd}}$ is sensitive to the acclimation state of leaves (sun-blue-shade-halfshade-shade) and correlates roughly with $P_{\mathrm{n}}$, and there is a strong correlation with $F_{V} / F_{O}$. The fluorescence decrease from $F_{P}$ to $F_{S}$ depends on several factors that have not yet been characterized completely, complicating the physiological interpretation of $R_{\mathrm{fd}}$ values (Roháček 2002).

Recently, fast Chl $a$ fluorescence kinetics and the JIP test (Strasser et al. 2000) have become popular for rapid screening of stress effects. The measurements are as simple as $F_{V} / F_{M}$ measurements and provide additional information on the photochemistry of PSII and the photosynthetic electron transport chain. Figure 8 shows the comparison of the response of three parameters $\left[F_{V} / F_{M}\right.$ measured by the PAM (Fig. 8a) and HandyPEA (Fig. 8b), $\Phi_{\text {PSII }}$ (Fig. 8a) and the Performance Index ( $P I_{\text {tot }}$ ) (Fig. 8b)] to decreasing leaf water content (RWC) in wheat. The $F_{V} / F_{M}$ level is quite insensitive to a decrease in RWC, whereas $\Phi_{\text {PSII }}$ and 
$P I_{\text {tot }}$ respond strongly to a RWC decrease, showing a similar dependence on RWC. Similar decreases have been observed under natural conditions during slowly advancing drought stress (Živčák et al. 2008b). The decrease in the Performance Index is associated with changes in Chl $a$ fluorescence transients and, by extension, changes in rate constants of individual electron transport steps and/or the state of the ETC that are detected by fast Chl $a$ fluorescence induction (reviewed, e.g., by Lazár 1999; Strasser et al. 2004). The simplicity of the fast Chl $a$ measurements and automated analysis have sometimes led to misapplication. Analysis should be supported by parallel measurements using other methods, and the restrictions of the JIP test analysis should be kept in mind (is the $F_{M}$ level still reached, is the $F_{O}$ truly measured, is the electron transport chain fully reoxidized?).

\section{Question 26: Can Chl $a$ fluorescence be used for high-temperature stress tolerance comparisons?}

Photosynthesis is very sensitive to high temperatures and can be partially or even completely inhibited before other stress symptoms are observed. High temperatures affect photosynthesis by their effect on the rates of chemical reactions and on the structural organization of the photosynthetic apparatus (Pastenes and Horton 1996).

Photosynthetic $\mathrm{CO}_{2}$ assimilation decreases at moderately high temperature levels (up to $38{ }^{\circ} \mathrm{C}$ ) due to thermal inactivation of Rubisco activase, the enzyme that removes inhibiting molecules from the active site of Rubisco (Feller et al. 1998; Law and Crafts-Brandner 1999; Salvucci and Crafts-Brandner 2004). This decrease is reversible and leads to a decrease in the linear electron transport rate, which can be detected using the saturating pulse method.

Direct impairment of PSII occurs when the leaf temperature reaches $\sim 40{ }^{\circ} \mathrm{C}$ and higher. This may be due to high-temperature-induced changes in the properties of thylakoid membranes (Sharkey and Zhang 2010; Yamauchi and Sugimoto 2010), the dissociation of the manganese-stabilizing protein from the PSII reaction center complex and the release of Mn atoms (Yamane et al. 1998). The Mn cluster can be reconstituted (Ananyev and Dismukes 1996 and references therein); however, in the leaf this does not seem to happen and once the Mn cluster has fallen apart, PSII follows the same repair cycle as in the case of photoinhibition (Tóth et al. 2005b; Komayama et al. 2007).

Damage to PSII can be observed in slow Chl $a$ fluorescence kinetics using the saturating pulse method (e.g., Pastenes and Horton 1996), but the most efficient way is through measurements taken after a period of dark acclimation. The high-temperature effects on PSII photochemistry and high-temperature tolerance at the PSII level have frequently been characterized in terms of $F_{O}$ increases or $F_{M}$ decreases. However, the basal and maximum fluorescence values are rather variable between different samples, even under non-stressed conditions, and their use can, therefore, become a source of uncertainty.

The maximum quantum yield, $F_{V} / F_{M}$, is the most frequently used measure of direct high-temperature effects on PSII. This parameter is based on the assumption that $F_{O}$ is measured for open RCs $\left(Q_{A}\right.$ fully oxidized) and $F_{M}$ for closed RCs ( $Q_{A}$ fully reduced). Under high-temperature conditions, $F_{O}$ can slightly increase as high temperatures enhance the process of chlororespiration, leading to a partial reduction of $Q_{A}$ in the dark due to a more reduced PQ pool (Sazanov et al. 1998). In high-temperature-stressed samples with a large population of PSII with an impaired oxygen-evolving complex (OEC), electron transport rate is lower and it takes longer to reach the maximum fluorescence intensity. In high-temperature treated samples, FNR may be activated before $F_{M}$ is reached and then the measured $F_{M}$ does not relate to a fully reduced ETC-a prerequisite for $F_{M}$ determination under non-inhibited conditions-causing an underestimation of $F_{M}$ (Tóth et al. 2005a, 2007a). Both the overestimation of $F_{O}$ and underestimation of $F_{M}$ lead to $F_{V} / F_{M}$ underestimation and therefore overestimate the temperature effect. In addition, the observed change in the $F_{V} / F_{M}$ value is related to a loss of electron donation capacity and not to a change in the PSII quantum yield.

Rate constants or some parameters derived from fast $\mathrm{Chl}$ $a$ fluorescence induction show a greater sensitivity to high temperatures than conventional fluorescence parameters such as $F_{V} / F_{M}$. The typical "visual" symptom of PSII high-temperature injury is the appearance of a new peak within the fluorescence induction curve at approximately $300 \mu$ s (denoted as the $\mathrm{K}$ step). This is frequently accompanied by a slowdown of the $J-I$ rise and by an increase in the amplitude of the $I-P$ rise (Fig. 9). The K step appears as a consequence of a high-temperature-induced destruction of the Mn cluster in a considerable fraction of PSII RCs (Srivastava et al. 1997; Tóth et al. 2005a).

The $K$ step has only been described in response to hightemperature stress or manganese deficiency, and its occurrence is thus a very specific symptom of high-temperature-induced damage of PSII. This is clearly illustrated in Fig. 10a, where $V_{K} / V_{J}$ values derived from fluorescence kinetics recorded on wheat plants subjected to progressive drought stress and a high-temperature treatment are shown. It is evident that even very strong drought stress did not induce any increase in $V_{K} / V_{J}$, whereas short periods of high temperatures caused substantial increases in this parameter. High-temperature stress responses vary considerably when 


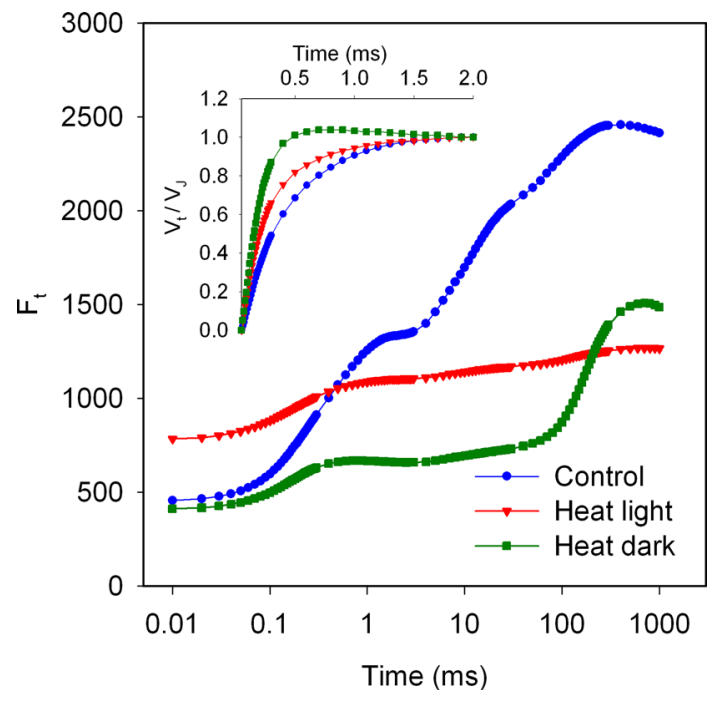

Fig. 9 Comparison of the effect of high-temperature stress applied in the light and in darkness on the OJIP kinetics. In the inset the Chl $a$ fluorescence transients double-normalized between $O$ and $J$ are shown (Živčák and Brestič, unpublished data)

data from a large collection of wheat genotypes of diverse provenance are used (Brestič et al. 2012).

The $K$ step is much more apparent if the leaves are heated in the dark (Fig. 9), since light causes a constant oxidation of the $\mathrm{Mn}$ cluster and therefore prevents the super-reduction of the Mn cluster, which is responsible for the disintegration of the Mn cluster in the absence of extrinsic proteins (e.g., Beck and Brudvig 1987). As a consequence, the $K$ peak is much lower under such conditions (Fig. 9). RCs that lack a Mn cluster are very sensitive to light (e.g., Blubaugh and Cheniae 1990) and, therefore, quickly become photodamaged. High-temperature susceptibility of PSII differs depending on species, age, physiological status, acclimation level, etc. The ability of PSII to tolerate high-temperature stress is termed "PSII thermostability" and is clearly demonstrated by the dependence of basal fluorescence on temperature $\left(F_{O^{-}} \mathrm{T}\right.$ curve), introduced by Schreiber and Berry (1977). The $F_{O^{-}}$ $\mathrm{T}$ curve method is based on a continuous increase of sample temperature during which the $F_{O}$ value is continuously recorded. The critical temperature $T_{\mathrm{C}}$ represents the temperature at which $F_{O}$ starts to increase steeply (Fig. 10b). As shown by Ducruet (1999), this increase is due to a shift in the $\left(Q_{A} Q_{B}\right)^{-}$equilibrium toward $Q_{A}$. The critical temperature for different species may vary considerably, with the critical temperature ranging from $42{ }^{\circ} \mathrm{C}$ up to more than $50{ }^{\circ} \mathrm{C}$ (Froux et al. 2004). It is known that increasing the saturation level of membrane lipids increases the tolerance to high temperatures (Murakami et al. 2000) and differences in this respect between plant species may explain in part the observed variability.
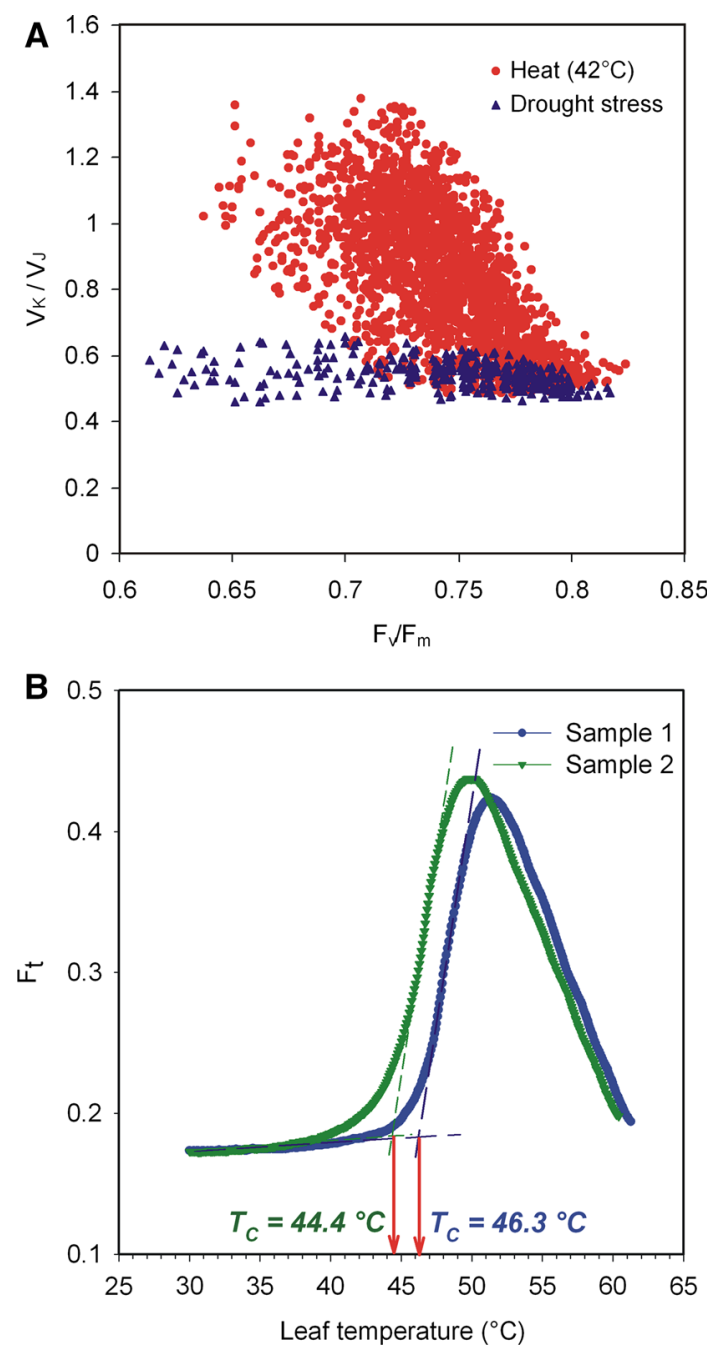

Fig. 10 High-temperature stress effects. a The parameter $V_{K} / V_{J}$ as a function of the parameter $F_{V} / F_{M}$ used here as a measure for the severity of the treatment. Heat stress induces a significant increase in the value of the parameter $V_{K} / V_{J}$, whereas drought stress has no effect on this parameter. b. Demonstration of the critical temperature $\left(T_{\mathrm{C}}\right)$ : gradually increasing the leaf temperature leads above a certain critical temperature to an increase of the $F_{O}$ value. $T_{\mathrm{C}}$ is determined as indicated in $\mathbf{b}$; it differs between photosynthetic samples and may be used as an indicator for adaptation or tolerance to heat (Živčák and Brestič, unpublished data)

Analogous to the continuous $F_{O}$ measurements, the exposure of fresh leaf samples to a single temperature (the graduated temperature approach) (Živčák et al. 2008c; Brestič and Živčák 2013) provides more detailed information about high-temperature effects on PSII photochemistry and enables the estimation of the $T_{\mathrm{C}}$ for $F_{O}$. Critical temperatures represent the point at which a severe disorganization of structure and loss of main functions occur. Hence, the estimated critical temperature can occur above the "physiologically relevant" temperature range, reaching temperatures as high as $50^{\circ} \mathrm{C}$. This estimate is non-physiological, as in most cases leaves in a field are not 
typically exposed to $50{ }^{\circ} \mathrm{C}$ or more. On the other hand, the use of graduated temperatures with fast $\mathrm{Chl} a$ fluorescence not only gives a real-life $T_{\mathrm{C}}$ measurement, but also enables the calculation of other parameters. A broad study on 30 field-grown wheat genotypes demonstrated that the $K$ step occurred even at temperatures $4-5{ }^{\circ} \mathrm{C}$ lower than the steep $F_{O}$ increase (Brestič et al. 2012). Moreover, the capacity to increase thermostability of the $\mathrm{K}$ step was higher than for the $F_{O}$ increase. High-temperature acclimation can occur quite quickly. Lazár et al. (1997a, b) observed a shift of the temperature at which the $\mathrm{K}$ peak appeared $\left(T_{\mathrm{K}}\right)$ by $3{ }^{\circ} \mathrm{C}$ (from 47 to $50{ }^{\circ} \mathrm{C}$ ) following the incubation of barley leaves for $2-3 \mathrm{~h}$ at $35{ }^{\circ} \mathrm{C}$. Using possibly the same acclimation data set Lazár and Ilík (1997) observed an approximately linear relationship between $T_{\mathrm{C}}$ and $T_{\mathrm{K}}$. However, $T_{\mathrm{K}}$ increased more than $T_{\mathrm{C}}$ following acclimation and $T_{\mathrm{K}}$ was always higher than $T_{\mathrm{C}}$. This contradicts somewhat the above-mentioned observation of Brestič et al. (2012).

PSII thermostability is only a small part of overall hightemperature tolerance. The reversible inactivation of Rubisco activase occurs already at considerably lower temperatures than PSII inactivation. However, inactivation of the donor side of PSII takes considerably longer to repair than re-activation of Rubisco activase. As a consequence, PSII donor side inactivation may not so much play a role during a high-temperature episode; instead, it may have an effect on plant photosynthetic productivity in its aftermath. In any case, the application of fast $\mathrm{Chl} a$ fluorescence measurements with $F_{O}, F_{V} / F_{M}$ along with the $\mathrm{K}$ step determination appears to be an efficient approach for screening PSII thermostability, enabling rapid identification of high-temperature-resistant or high-temperature-sensitive samples.

\section{Question 27: Can variable Chl $\boldsymbol{a}$ fluorescence be used as a biomarker of environmental pollution?}

By using Chl $a$ fluorescence it is possible to detect the effects of environmental stressors (e.g., herbicides, ozone, trifluoroacetic acid, acid rain, many heavy metals) that disrupt photosynthesis (Judy et al. 1991; Lewis et al. 2001; Guidi et al. 2010; Chaudhary et al. 2013). Chl $a$ fluorescence-based methods have been applied in ecotoxicological studies to examine the effects of pollutants on algae and plants (Kumar et al. 2014). These methods have many advantages over existing bioassays, especially with regard to sensitivity, rapidity, and the non-destructiveness and non-invasiveness of the methodology. Depending on the stressor, changes can be detected before visible symptoms appear (Guidi et al. 1997, 2000; Popovic et al. 2003).

While it is commonly assumed that parameters linked to PSII electron transport are indicators for toxicity in plants (e.g., Perreault et al. 2010), it is important to specify which kind of parameter could be a good biomarker for a particular agent. For instance, in Lemna plants exposed to copper oxide nanoparticles, the effective PSII quantum yield $\left(\Phi_{\mathrm{PSII}}\right)$ is a reliable indicator for toxicity after $24 \mathrm{~h}$ of exposition, but $F_{V} / F_{M}$ remains unaffected (Perreault et al. 2010). However, upon long exposure of Lemna to a heavy metal such as $\mathrm{Cr}, F_{V} / F_{M}$ and $F_{\mathrm{V}} / F_{O}$ showed a clear dosedependent decrease (Reale et al. 2016). Similarly, $F_{V} / F_{M}$ can be used for the detection of several herbicides that inhibit linear electron transport (see Question 28) and petrochemicals that can contaminate natural waterways or reservoirs (Conrad et al. 1993; Ralph and Burchett 1998; Dorigo and Leboulanger 2001; Choi et al. 2012). The effective quantum yield has been found to be a sensitive indicator for certain heavy metals, herbicides and petrochemicals (Ralph and Burchet 1998; Haynes et al. 2000; Juneau et al. 2001; Marwood et al. 2001; Macinnis-Ng and Ralph 2003; Perreault et al. 2010; Wilson and Ralph 2012).

The tolerance to pollutants differs between plant species. Uptake reduction, compartmentalization or differential detoxification are strategies that have been demonstrated in plants (Hall 2002). For every bioassay it is, therefore, important to choose plant or algal species that are sensitive to the pollutant or mixture of pollutants of interest. In the case of crops, the comparison of sensitive and tolerant cultivars can also be interesting (e.g., Calatayud et al. 2002; Degl'Innocenti et al. 2002).

In summary, for a successful bioassay, the choice of a species sensitive to the pollutant and of a Chl $a$ fluorescence parameter that is affected by the pollutant in the concentration range of interest is critical (Choi et al. 2012). Taking these considerations into account, Chl $a$ fluorescence methods represent a more rapid and sensitive methodology than growth assays (Kvíderová 2010; Fai et al. 2007).

\section{Question 28: Can toxicity induced by different herbicides be identified?}

Screening for herbicide efficacy and plant sensitivity is usually a lengthy process. The experiments take up a lot of space, and the evaluation of results, either quantitatively or qualitatively, is normally completed more than a week after the treatment (Christensen et al. 2003). In this respect, the most straightforward category of herbicides is those that bind to the $Q_{B}$ site of PSII (e.g., DCMU, atrazine and phenolic herbicides like ioxynil or bromoxynil). Full inhibition of PSII by these herbicides raises $F_{J}$ to the $F_{M}$ level, and the percentage of increase between $F_{J}$ and $F_{M}$ can be easily quantified (cf. Lazár et al. 1997a, 1997b, 1998; Tóth et al. 2005b). However, as 
shown by Krieger-Liszkay and Rutherford (1998), the effect of these herbicides on the midpoint potential of $Q_{A}$ affects their working mechanism. DCMU increases the midpoint potential of $Q_{A}$, stabilizing the charge separation, whereas phenolic herbicides decrease the midpoint potential of $Q_{A}$ and destabilize the charge recombination (see also Question 13). The first type of herbicides reduces the probability that a charge recombination induces singlet oxygen, whereas the second type increases this probability. In other words, the extent of inhibition is only half the story.

The activities of a surprisingly large number of herbicides are directly or indirectly influenced by light (Hess 2000). Herbicides that catalyze the formation of reactive oxygen species (e.g., paraquat and diquat), block the synthesis of carotenoids directly or indirectly, inhibit protoporphyrinogen oxidase during $\mathrm{Chl}$ biosynthesis or inhibit glutamine synthetase in the nitrogen assimilation pathway can, therefore, also be detected using fluorometric methods (Fuerst et al. 1985; Kaňa et al. 2004; Merkell et al. 2004; Søbye et al. 2011). Paraquat, also known as methylviologen, can accept electrons from the FeS clusters of PSI in competition with Fd. In dark-adapted leaves this is observed as a suppression of the IP phase of the OJIP transient (e.g., Schansker et al. 2005). Herbicides binding to the $Q_{B}$ site do not necessarily affect the $F_{O}$ or $F_{M}$ values (Tóth et al. 2005b). However, the measuring light of a PAM instrument will strongly increase the measured $F_{O}$ value in samples in which the re-oxidation of $Q_{A}^{-}$is fully or partially inhibited. This effect decreases the calculated $F_{V} /$ $F_{M}$ value and allows the detection of such herbicides (Bolhar-Nordenkampf et al. 1989).

Glyphosate application leads to a depletion of free phosphate, leading to an inhibition of ATP synthesis, and isoxaflutole inhibits PQ synthesis. Søbye et al. (2011) showed that $F_{J}$ and the slopes at the $J$ and $I$ steps can be used to titrate the effects of the herbicides glyphosate and isoxaflutole as well as mixtures of these two compounds. However, looking at the OJIP transients shown in that paper, these parameters seem to reflect mainly the destruction of either the photosynthetic system or alternatively PSII, since the main effect was observed on the $F_{V}$ amplitude. The authors note that the fluorescence data obtained $48 \mathrm{~h}$ after the treatment gave the same information as the biomass measurements carried out 3 weeks after the treatment.

Special equipment for the detection of herbicides in microalgae was introduced by the laboratries of JeanClaude Duval and Claudia Büchel (Arsalane et al. 1993; Conrad et al. 1993). The principle is the same as that for the PAM and $F_{V} / F_{M}$ mentioned above, but the equipment was considerably more sensitive than the PAM. The equipment works with low-intensity-modulated light that does not lead to the induction of fluorescence in the absence of herbicide, leads to the induction of fluorescence as a function of the fraction of PSII RCs inhibited by herbicide.

Herbicides that inhibit the synthesis of amino acids or lipids have indirect effects on photosynthesis through their effect on the carbon metabolism or the stability of thylakoid membranes and may consequently alter PSII and PSI photochemical activity. Their effects on the fluorescence kinetics will only be apparent after a much longer exposure time than in the case of PSII-type inhibitor herbicides (Popovic et al. 2003). Olesen and Cedergreen (2010) and Yanniccari et al. (2012) have argued that the inhibition of $\mathrm{CO}_{2}$ assimilation is a better and more direct probe for glyphosate effects than $\mathrm{Chl} a$ fluorescence. This is true for OJIP transients, but it can be pointed out that a full Kautsky curve measured till the steady state is reached would also give information on the inhibition of $\mathrm{CO}_{2}$ assimilation. As noted by Yanniccari et al. (2012), the parameter ETR (a parameter determined for steady-state conditions) is a good probe for herbicides that have a kinetic effect on photosynthetic activity since fluorescence measurements are easier and faster than quantification of $\mathrm{CO}_{2}$ assimilation by IRGA.

Chl $a$ fluorescence imaging does not only allow the measurement of a whole plant or several small plants simultaneously, and it also makes it possible to follow the spread of herbicides in leaves or whole plants (see Lichtenthaler et al. 2005b). Herbicide-induced perturbations of plant metabolism have been detected using changes in the derived images of fluorescence parameters before any visual effects on growth were observed (Barbagallo et al. 2003). Konishi et al. (2009) made a three-dimensional analysis of the uptake of DCMU, in plants of Cucumis melo, in which the third dimension was the uptake time. This allowed the authors to show in detail how the herbicide, arriving in the leaf via the xylem, spread inside the leaf. Saura and Quiles (2009), using Chl $a$ fluorescence imaging, to compare the uptake of DCMU and paraquat in Chrysanthemum morifolium, Rosa meillandina and Spathiphyllum wallisii, showed (1) that paraquat, an herbicide acting on the acceptor side of PSI, can also be monitored by Chl $a$ fluorescence imaging and (2) that the more water-soluble paraquat affected the leaves more homogeneously than DCMU. Muller et al. (2008) showed how a combination of the Maxi imaging PAM, black 96 well plates and Chlorella vulgaris and Phaeodactylum tricornutum as biosensors can be used as a rapid and inexpensive bioassay for herbicides in, e.g., water samples.

Chl $a$ fluorescence imaging has not only been used to detect the effects of herbicides on the photosynthetic performance of plants but also of algae as recently reviewed by Kumar et al. (2014). 


\section{Question 29: Can fluorescence parameters be used for QTL studies?}

QTLs or quantitative trait loci refer to a location on a chromosome coding for one or more genes that affect a certain characteristic or process, e.g., stress tolerance or sensitivity. It is likely that, e.g., drought stress tolerance is affected by many genes that can be found on several chromosomes. The word quantitative refers to the fact that the genes linked to a QTL only have partial control over a characteristic. With respect to photosynthesis there is one more peculiarity. QTLs are located in the nuclear genome, whereas many important photosynthetic genes are found in the chloroplast genome.

Several studies have been carried out that had as goal to identify QTLs related to fluorescence parameters (see Question 30 for a discussion of a rational choice of parameters for such studies). There are two main approaches to the identification of QTLs, but for the study of QTLs related to fluorescence parameters only one of them, association mapping, has been used (see Flood et al. 2011 for a discussion of linkage mapping). Czyczyło-Mysza et al. (2013) used for their study 94 daughter lines from the cross of two wheat varieties: Chinese spring $\times$ SQ1. For these daughter lines the authors determined the following parameters: $F_{V} / F_{M}, P I_{\text {abs }}$ (performance index), ABS/CSm, $\mathrm{TRo} / \mathrm{CSm}, \mathrm{ETo} / \mathrm{CSm}, \mathrm{DIo} / \mathrm{CSm}, \mathrm{RC} / \mathrm{CSm}, \mathrm{Chl} a+b$, SPAD, Car, DWP (dry weight per plant), GWE (grain yield of the main stem), YP (grain yield per plant).

Separately, a genetic map was made based on the DNA of 90 lines. This resulted in a Chinese spring $\times$ SQ1 genetic map defined by 1039 genetic markers of which 472 were derived from their study. In addition, 165 genes related to the photosynthetic light reactions, pigment metabolism.

The authors then tested, which of the studied parameters were linked, in the sense that they were inherited together in the different daughter lines. By studying the extent of linkage between the parameters and the genetic markers, the genes controlling the variability in the studied parameters could be assigned to areas on particular chromosomes. Czyczyło-Mysza et al. (2013) observed that the productivity traits were not consistently correlated with any of the fluorescence or pigment traits.

Similar studies were carried out by several other groups. Stamp and coworkers published several QTL studies related to chilling tolerance in maize. Fracheboud et al. (2002) studied 233 recombinant inbred lines derived from a cross between a drought-tolerant and a drought-sensitive maize variety grown at 15 and $25{ }^{\circ} \mathrm{C}$. The authors screened the following parameters: $\mathrm{CO}_{2}$ assimilation, $\Phi_{\mathrm{PSII}}, F_{V} / F_{M}, F_{O}$ (rel.), stomatal resistance, $\mathrm{Chl} a \times b, \mathrm{Chl} a / b, \quad \beta-$ carotene/lutein and different carotenes/xanthophylls. In a second study, Fracheboud et al. (2004) screened: $F_{O}, F_{V} /$ $F_{M}, \Phi_{\mathrm{PSII}}, F_{V}^{\prime} / F_{M}{ }^{\prime}, q_{\mathrm{P}}, \mathrm{CER}\left(\mathrm{CO}_{2}\right.$ assimilation), SPAD, shoot DW, $N(\%)$ (nitrogen content). Apart from an assignment of several QTLs, these studies also yielded a lot of biological variability allowing the study of the relationship between different parameters. Yang et al. (2007) studied 150 recombinant inbred lines of the cross Hanxuan $10 \times$ Lumai 14 lines of wheat under drought and wellwatered conditions. The authors screened the following parameters: Chl content, $F_{O}, F_{M}, F_{V}, F_{V} / F_{M}, F_{V} / F_{O}$. The authors noted that for each of the two conditions studied different QTLs were found. An observation that is made in several other studies as well. Yin et al. (2010) studied 184 recombinant inbred lines of crosses of the soybean varieties Kefeng no 1 and Nannong1138-2. The authors used the following parameters for screening: TRo/ABS $\left(F_{V} / F_{M}\right.$ JIP test), ETo/TRo, REo/ETo, ABS/RC, $P I_{\text {abs }}, F_{V} / F_{M}$ (PAM), $F_{V}^{\prime} / F_{M}{ }^{\prime}, q_{P}, \Phi_{\mathrm{PSII}}, P_{\mathrm{n}}\left(\mathrm{CO}_{2}\right.$ assimilation rate $)$. The authors planted at different times in order to be able to measure all the plants at the same age. Remarkable was the observation that the $F_{V} / F_{M}$ measured with the HandPEA did not yield the same results as the $F_{V} / F_{M}$ measured with a PAM instrument. It is possible that the authors erroneously used the 50- $\mu$ s point for the $F_{O}$ value of the OJIP measurements, but it may also suggest that there were methodological problems. Šimić et al. (2014) studied 205 recombinant inbred lines of the cross of the maize lines B73 $\times$ M017 in four environmental conditions differing in soil moisture levels. The authors used the following parameters for screening: TRo/ABS, ETo/TRo, $\mathrm{ABS} / \mathrm{RC}, \mathrm{TRo} / \mathrm{RC}, \mathrm{ETo} /$ RC, DIo/RC, TRo/DIo, ETo/(TRo - ETo), $P I_{\text {abs. }}$. The authors identified 10 significant QTLs, but also observed that different QTLs were found for different environmental conditions.

With respect to the JIP test parameters chosen in the above-mentioned studies, it can be noted that most of them have not been characterized physiologically. See the next question for a discussion about the choice of parameters for this type of study.

Fracheboud et al. $(2002,2004)$ tried to link their QTLs to candidate genes. In most of the other cited studies the link with the physiology of the studied plants is less evident.

\section{Question 30: Which parameters to choose for QTL studies?}

Fluorescence parameters reflect an underlying process or processes. If we would assume, for example, that a decrease in PSI content during stress is a marker for 
oxidative damage, then the resistance of the IP amplitude to stress could be a good candidate for a QTL study. Although the meaning of changes in the ratio between the amplitude of the photochemical phase and the amplitude of the thermal phase has not been established, it probably reflects a fundamental property of the chloroplast and may, therefore, be another candidate. Another parameter could be the normalized area [the parameter Sm of the JIP test (Strasser et al. 2004)], or even the normalized area split in parts (area between $F_{M}$ and the OJ, JI and IP rise, respectively; see Tóth et al. 2007b and Question 16) that give information on the ratio between PSII and the number of electron acceptors downstream (PQ pool and acceptorside PSI). For the $q_{\mathrm{E}}$ several genes have already been identified (Jung and Niyogi 2009) and it may, therefore, be a less interesting parameter for a QTL study. The parameter $q_{\mathrm{P}}$, as a rough indicator of the balance between excitation pressure and electron flow, in response to a certain stress may be another candidate parameter to screen for stress resistance genes.

Parameters that have not been characterized physiologically are less rational choices for QTL studies. Many JIP test parameters are only used conceptionally and have so far not been characterized physiologically in a proper way. Quite a few of the JIP test parameters mentioned in the previous question belong to this category of conceptional parameters. Other parameters are so fundamental that they are unlikely to change much. The quantum yield of PSII has been shown to be sensitive to antenna size (Wientjes et al. 2013a), but, apart from that, is essentially invariable (see Question 6). On the other hand, the parameter $F_{V} / F_{M}$ can be used to probe the resistance to photoinhibition.

QTL studies are, in principle, not so different from mutant screening. If you do not have a well-thought-out strategy, it is unlikely that you will obtain interesting information.

In summary, in our opinion, QTL studies can yield more interesting information if a more rational approach, making better use of our knowledge of the meaning of fluorescence parameters, is applied.

\section{Question 31: Can Chl a fluorescence measurements/parameters be used for crop improvement?}

As discussed in response to several questions in this paper (e.g., 25 and 26), Chl $a$ fluorescence can be used to monitor environmental stress. Therefore, fluorescence parameters can, in principle, be used as selection tools in plant breeding programs and for analyzing genotype-environment interactions (Araus et al. 1998; Kalaji and Pietkiewicz 2004). In quite a few studies this approach has been advocated (Greaves and Wilson 1987; Baker and Rosenqvist 2004; Kalaji and Pietkiewicz 2004).

There are a few points that should be considered: (1) It is important to obtain $\mathrm{Chl} a$ fluorescence-related traits showing a high correlation with yield or plant performance in addition to Chl $a$ fluorescence-related traits that are specific for resistance to the stress of interest; (2) the measurements should cause only minimal perturbations in growth conditions: The shorter the measurements the better; (3) short measurements that are easy to carry out, to allow the accumulation of many measurements in a short time-plant characteristics change during growth in response to both age and environmental factors. To allow comparisons between different cultivars, varieties or crosses of all plants of interest, measurements have to be made within a short time interval. With respect to point (1), it may be noted that our knowledge of plant stress responses is often too limited to decide with certainty which trait will improve both stress resistance and plant yield. For example, in the case of photoinhibition it has been argued widely that this represents damage. More recently, however, it has been suggested that the inactivation of PSII by light (reversible damage) prevents damage to PSI (largely irreversible damage) (Grieco et al. 2012; see Question 8). Breeding for plants with PSII that is no longer inactivated by light could, therefore, have catastrophic consequences for the survival chances of such plants. Another example of such a conflict of interest is the observation that increasing the synthesis of enzymes that scavenge ROS may make the plant more resistant to abiotic stress, but, at the same time, more sensitive to biotic stress, because it weakens programmed-cell-death-based defense mechanisms against pathogens (Mittler 2002).

Thus, two Chl $a$ fluorescence-related traits have to be used to screen for crop improvements: (1) processes that are closely correlated with plant performance, which can be monitored by Chl $a$ fluorescence and (2) Chl $a$ fluorescence-related traits that correlate with the stress of interest and are under genetic control and can be genetically manipulated.

In the field, plants often are subjected to several types of biotic or abiotic stresses during the growing season. If two or three types of stress interact, the response of the plant may differ considerably from the response to individual stresses. This complicates the analysis of data for breeding programs directed at a particular stress. It should be noted that the growth conditions of horticultural plants grown in greenhouses can, in this respect, be much better controlled.

If stress combinations are common, it is also possible to target such a combination of stresses. Rosyara et al. (2010) looked at the combination of high-temperature stress and spot blotch in wheat. They observed that the parameters $F_{V} / F_{M}$, Chl content (SPAD measurements) and the 
parameter canopy temperature depression (measured using infrared thermography) showed a better correlation with plant yield (e.g., thousand kernel weight) than the leaf area affected by spot blotch.

The inheritance of $\mathrm{Chl} a$ fluorescence features in different stress environments indicates typical quantitative traits determined by multiple QTLs (Fracheboud et al. 2004; Hund et al. 2005; Yang et al. 2007; Guo et al. 2008; Kalaji and Guo 2008; Kiani et al. 2008; Zhang et al. 2010; Czyczyło-Mysza et al. 2013; Anithakumari et al. 2012). Depending on the complexity of the processes associated with a trait, the number of QTLs and their effect size differ.

Although the technical quality of Chl $a$ fluorescence measurements and the efficiency with which they can be carried out have increased over the years, there still remain many basic interpretation issues of which quite a few are mentioned here and in Kalaji et al. (2014a). Chl $a$ fluorescence analysis is now rapid, sensitive, non-destructive and relatively cheap (Misra et al. 2001a, b, 2006; Kalaji et al. 2012a). A discussion issue remains under which conditions Chl $a$ fluorescence measurements can be used for early detection of stress and which parameters are subsequently the best tools. Chl $a$ fluorescence can be measured on whole tree canopies down to single cells or even chloroplasts (Malenovsky et al. 2009; Snel and Dassen 2000). It has to be noted though that for imaging applications, the time resolution is considerably lower than for point measurements and homogeneous illumination of the measured leaves or plants remains a concern for kinetic fluorescence measurements. Also, for tree crown measurements, the relatively large distance between measuring equipment and photosynthetic sample imposes considerable limitations on the Chl $a$ fluorescence parameters that can be measured (Malenovsky et al. 2009).

One of the most important problems associated with the use of Chl $a$ fluorescence parameters is the heterogeneity of photosynthetic samples. Different factors such as senescence (Wingler et al. 2005), injury/wounding (Quilliam et al. 2006), microbial infection (Scharte et al. 2005; Bonfig et al. 2006; Berger et al. 2007), leaf water status (Meyer and Genty 1999; Nejad et al. 2006), photosynthetic induction (Meyer and Genty 1999), spatial gradients in thylakoid differentiation (Pantaleoni et al. 2009), chilling (Hogewoning and Harbinson 2007) and ozone exposure (Leipner et al. 2001) can cause photosynthetic heterogeneity. This type of photosynthetic heterogeneity is difficult to capture with non-imaging fluorescence techniques. Recent advances in fluorescence imaging technology has turned it into an important tool for resolving spatial heterogeneity of leaf photosynthesis (Nedbal and Whitmarsh 2004; Oxborough 2004). But even when using imaging techniques, it is not easy to quantify the effect of a pathogen infection on the photosynthetic capacity of a leaf based on Chl $a$ fluorescence measurements alone. In such cases, it would probably be necessary to calibrate such measurements using, for example, $\mathrm{CO}_{2}$ assimilation measurements that yield absolute activities.

For a breeder it is important to measure all plants in a breeding program in a short time to be able to compare the different crosses (see above). However, it is also possible to use imaging or even remote sensing methods depending on the question whether the chosen parameter(s) can be determined using such techniques. Overall, the measurement of Chl $a$ fluorescence can be helpful for breeding purposes if the questions "what to measure" and "how to measure it efficiently" can be resolved.

\section{Question 32: Are machine learning methods useful for the analysis of $\mathrm{Chl} a$ fluorescence induction curves?}

Prompt fluorescence (PF) induction curves reflect energy and electron transfer processes in thylakoid membranes following a dark-to-light transition of dark-adapted photosynthetic samples. Analyzing the shapes of the induction transient, the absolute values of the fluorescence signal at different times $\mathrm{F}_{t}$ during induction (OJIP), their relative amplitudes, normalized to the variable part of the signal, or difference curves reflecting stress effects, we can study different sites of the photosynthetic electron transport chain and monitor stress effects on them. Stress often affects not only specific sites of the photosynthetic structure or specific reactions, but modifies a wide range of cell structures (which may not all be directly related to the photosynthetic apparatus) and change their function. This may have secondary (indirect) effects on photosynthetic processes. These effects can be detected looking at changes in the "fingerprint" - a term also used by Tyystjärvi et al. (1999) — formed by the constellation of parameters derived from OJIP transients or by studying the modified shape of the fluorescence rise kinetics. This "stress response" often contains hidden information concerning the stress type, a specific plant tolerance to the applied stress, and other important and interesting information related to the plant as whole and that, at first sight, is not directly connected to the photosynthetic apparatus of the plant.

The identification of such kinds of hidden information is possible through additional secondary fluorescence data processing, using methods of artificial intelligence that allow the analysis of large data sets, the amount, precision and complexity of which cannot not be efficiently analyzed by traditional methods (Samborska et al. 2014). Here, the analysis of OJIP transients is discussed. In an earlier series of articles, Tyystjärvi and coworkers tried to apply similar artificial intelligence methods to the analysis of 
fluorescence data induced by a sequence of different types of illumination (low light intensity, saturating pulse, farred, etc.) in order to identify plant species (Tyystjärvi et al. 1999; Keränen et al. 2003; Codrea et al. 2003). Simulating OJIP transients is another approach to mine the information contained in OJIP transients, which is not further discussed here (reviewed by Lazár and Schansker 2009).

Machine learning methods, like artificial neural networks (ANNs) or self-organizing maps (SOM), are powerful tools of Chl $a$ fluorescence data analysis. They enable us to quickly classify the different responses of plants to environmental stress by (1) finding different shapes of $\mathrm{Chl}$ $a$ fluorescence induction curves, (2) determine the most important $\mathrm{Chl} a$ fluorescence parameters or points on the Kautsky curve that differentiate them. Moreover, we can also (3) predict the values of other environmental or physiological variables on the basis of Chl $a$ fluorescence data (Goltsev et al. 2012).

\section{Reducing data complexity}

For an analysis, we only need the table of points of Chl $a$ fluorescence induction curves, obtained from fluorimeters, or the table of measured/calculated Chl $a$ fluorescence parameters. Chl $a$ fluorescence data usually show noisy patterns of many variables and parameters cluttered on the charts. By using multivariate analyses similar to principal component analyses (PCAs) we can reduce the large set of Chl $a$ fluorescence variables to the few most informative ones (Legendre and Legendre 2012; Goltsev et al. 2012). This way, we can also detect the main trade-offs among $\mathrm{Chl} a$ fluorescence parameters and remove errors from the data set.

\section{Relating Chl a fluorescence parameters to environmental or physiological processes}

Artificial neural networks are designed to perform analyses that relate the selected $\mathrm{Chl} a$ fluorescence parameters (input) and output variables through a network of interconnected cells called neurons in a learning process in the same way the human brain works (Goltsev et al. 2012). The typical objective of ANNs is to find the Chl $a$ fluorescence parameters that characterize the "stressed" or "unstressed" plants (Kalaji et al. 2014b) or to enable us to predict values [e.g., water content in plant tissue (Goltsev et al. 2012), or predict the plant biomass].

\section{Visualization and classification}

Self-organizing maps (SOM) are used as a data mining and visualization method for complex data sets. SOM is a type of neural network and can be seen as a nonlinear form of
PCA. This method can also be used for the classification of fluorescence induction curves measured with portable fluorimeters by displaying the groups of $\mathrm{Chl} a$ fluorescence parameters in an easy to understand form in a two-dimensional plane (Maldonado-Rodriguez et al. 2003).

Example 1 In Fig. 11a, the results of a PCA analysis of $54 \mathrm{Chl} a$ fluorescence parameters randomly sampled from a large set of Chl $a$ fluorescence measurements measured on Brachypodium pinnatum (a grass) are shown. The grass grew in meadows, and its density increased with age. The relative variable fluorescence at the $I$ step $\left(V_{\mathrm{I}}\right)$ decreases, when we move along the first PCA axis (Dim1), from the left to the right side of Fig. 11a. At the same time an increase of the parameters that, according to the JIP test, represent specific energy fluxes expressed per active PSII
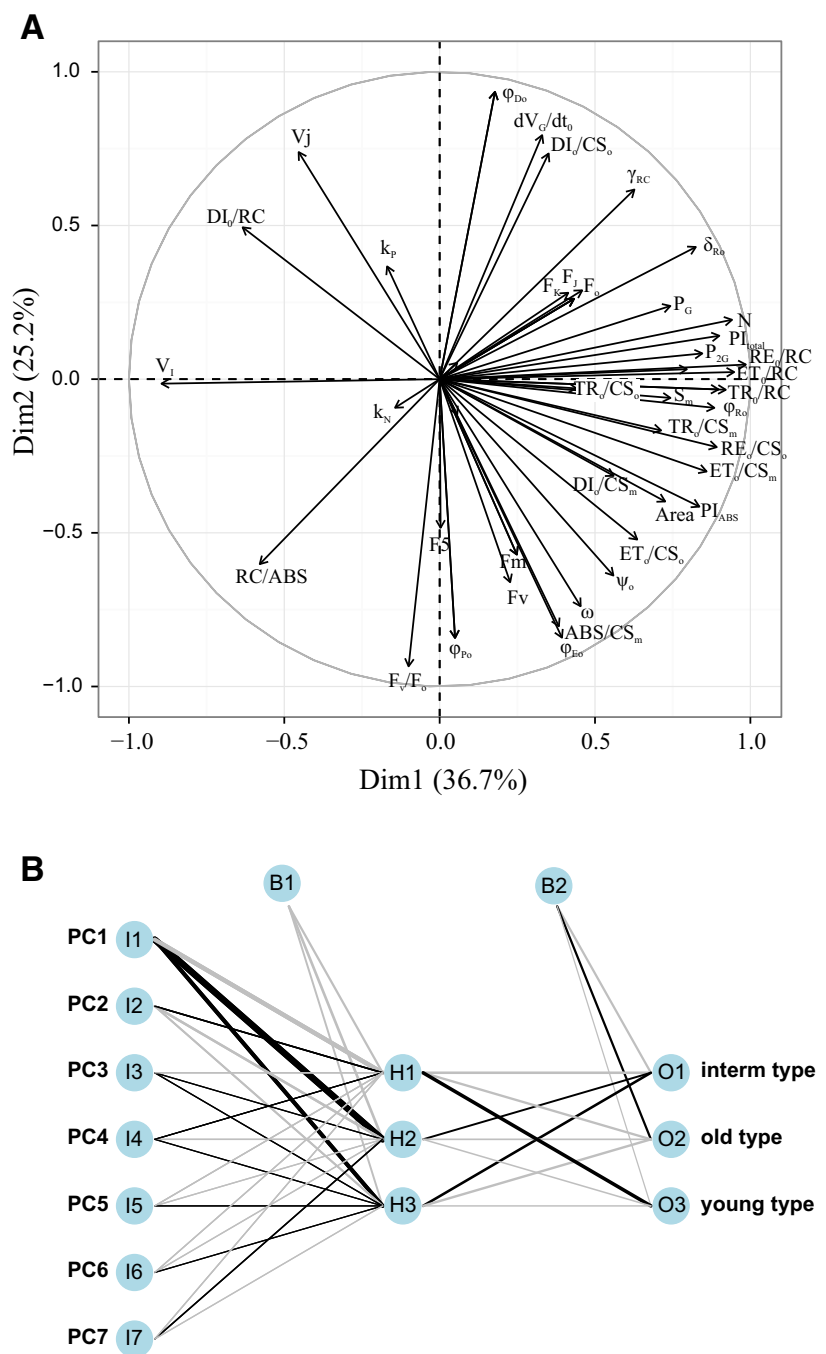

Fig. 11 Machine learning methods. a principal component analysis (PCA) of $54 \mathrm{Chl} a$ fluorescence parameters derived from a study on the effect of the density of grass on OJIP measurements. b Artificial neural network (ANN) analysis of the same data set. For further details see Question 32 (Bąba, unpublished data) 
reaction center $\left(\mathrm{TR}_{O} / \mathrm{RC}, \mathrm{ET}_{O} / \mathrm{RC}, \mathrm{RE}_{O} / \mathrm{RC}\right)$ is observed. When we move along the second PCA axis (Dim2), from bottom to upper side of Fig. 11a, the increase of the values of the parameters $F_{\mathrm{V}} / F_{O}$ and maximum quantum yield of primary PSII photochemistry $\left(\phi_{\mathrm{Po}}\right)$ can be seen. In this way we find the four most important parameters (instead of the initial 54), which describe the state of the photosynthetic apparatus, related to the studied increase in the stand density of grass.

Example 2 In Fig. 11b, the artificial neural network (ANN) analysis of Chl $a$ fluorescence of the same data set is shown. As in the previous analyses we performed the PCA and retained the first 7 axes (the two most important axes are related to fluorescence parameters from Example 1). The ANNs find the relationships between these parameters and the plants of different age growing in grasslands. In other words, the analyses allow the comparison of the responses of the photosynthetic apparatus to the condition of these three plots.
Example 3 Unsupervised self-organizing maps (SOM) using another population of plants growing under stressed (drought stress) and unstressed (well-watered) conditions. The pattern of differences in Chl $a$ fluorescence parameters $\left(F_{0}, F_{1}, \mathrm{TR}_{0} / \mathrm{RC}, \mathrm{ET}_{0} / \mathrm{RC}, \mathrm{RE}_{0} / \mathrm{RC}, \mathrm{Sm}, \phi_{\mathrm{Po}_{\mathrm{o}}}\right)$ is easily detectable (SOM; Fig. 12).

Examples of successful use of machine learning methods for Chl $a$ fluorescence analysis from the literature are:

1. Taxonomic classification of plant species based on Chl $a$ fluorescence parameter data using an artificial neural network (Kirova et al. 2009)

2. Determination of relative water contents (RWCs) in leaves based on PF, DF and MR820 signals $(=820 \mathrm{~nm}$ reflection signal) or calculated JIP parameters (Goltsev et al. 2012)

3. Prediction of the yield of wheat based on selected factors in wheat crop production with self-organizing maps (Pantazi et al. 2014)
Fig. 12 Example of a selforganizing map (SOM) based on a set of OJIP measurements of unstressed and droughtstressed plants. For further details see Question 32 (Baba, unpublished data)

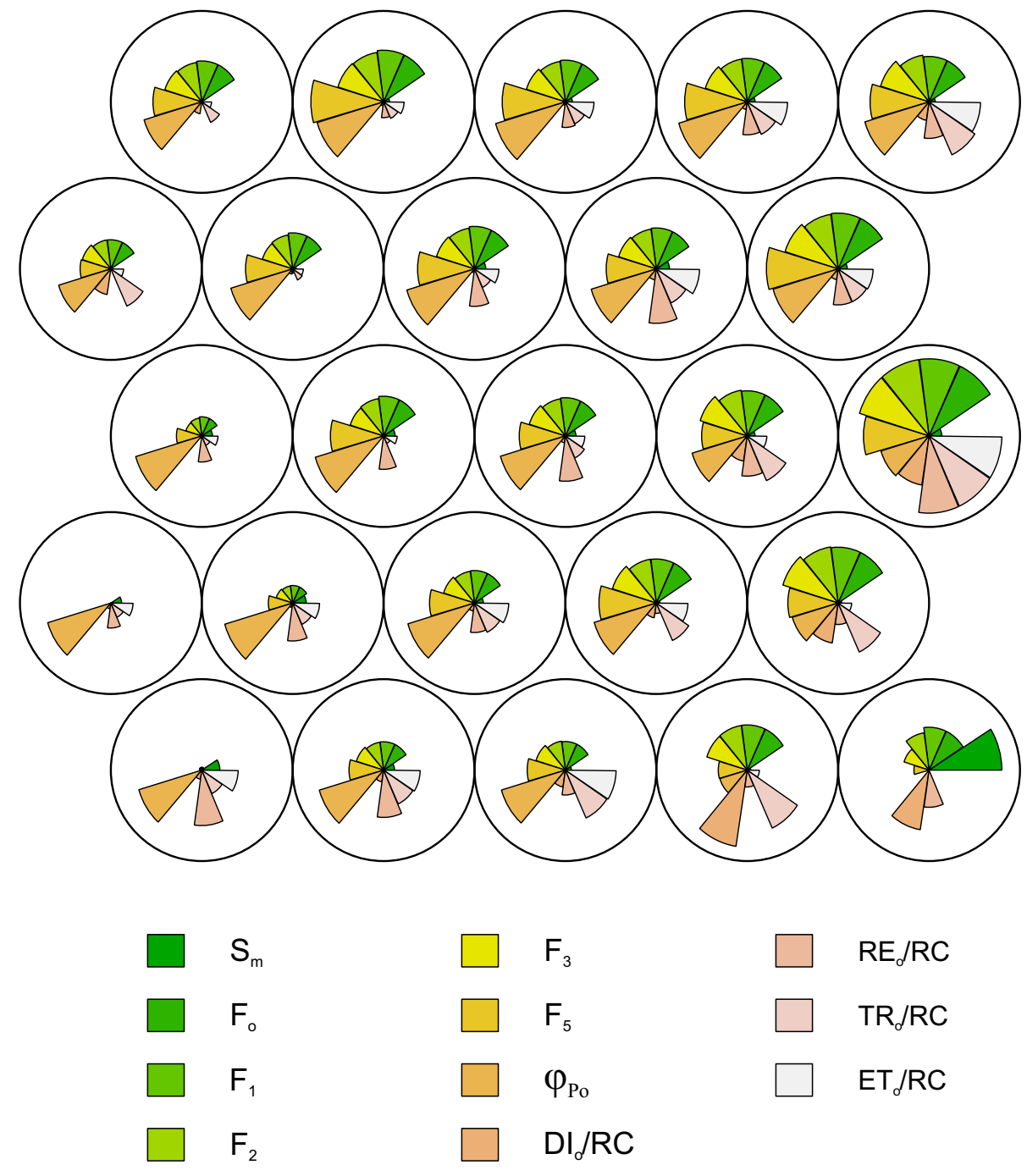


How to perform the analyses? All analyses presented above are implemented using statistical packages, for example Statistica (StatSoft Inc. 2011) or SAS (SAS Enterprise Miner; SAS Institute, Cary, NC). Neural network analyses can also be performed in MATLAB with the Neural Network Toolbox (MathWorks).

\section{Question 33: Which are the most useful books about Chl $a$ fluorescence?}

There are not many books dealing with $\mathrm{Chl} a$ fluorescence. Since the 1980s there have been two classic references: "Light Emission by Plants and Bacteria" (1986), edited by Govindjee, Amesz and Fork; and "Applications of Chlorophyll Fluorescence in Photosynthesis Research, Stress Physiology, Hydrobiology and Remote Sensing" (1988), edited by Lichtenthaler. More recently, Laisk and Oja (1998) published "Dynamics of Leaf Photosynthesis on Dynamic Measurements of Photosynthesis in Leaves" and DeEll and Toivonen (2003) published "Practical Applications of Chlorophyll Fluorescence Science in Plant Biology." Further there are three books in the series Advances in Photosynthesis and Respiration that contain much information on Chl $a$ fluorescence: "Chlorophyll a Fluorescence: a Signature of Photosynthesis" (2004), edited by Papageorgiou and Govindjee, "Photosynthesis in Silico: Understanding Complexity from Molecules to Ecosystems" (2009), edited by Laisk, Nedbal and Govindjee and "Non-photochemical Quenching and Energy Dissipation in Plants, Algae and Cyanobacteria" (2014), edited by Demmig-Adams, Garab, Adams and Govindjee. A recent addition is "Applications of Chlorophyll Fluorescence in Understanding Plant Performance" (2016), edited by Kalaji et al. In addition, several review papers have been published: Krause and Weis (1991), Dau (1994), Govindjee (1995), Rohácek and Barták (1999), Maxwell and Johnson (2000), Roháček (2002), Lazár (2006), Logan et al. (2007), Baker (2008), Henriques (2009), Allakhverdiev (2011), Gorbe and Calatayud (2012), Misra et al. (2012), Kalaji et al. (2012b), Murchie and Lawson (2013) and Lazár (2015).

\section{Concluding remarks}

Chlorophyll $a$ fluorescence is a simple, non-invasive, nondestructive, quick and relatively easy way to determine the efficiency and activity of the photosynthetic electron transport chain. However, as this paper and the previous paper (Kalaji et al. 2014a) demonstrate, the interpretation of fluorescence measurements demands knowledge. Both knowledge of the sample (e.g., do I still measure the true
$F_{O}$ or true $F_{M}$ ) but also of all the photosynthetic processes that can affect the fluorescence intensity, are important. Once these two requirements are met, $\mathrm{Chl} a$ fluorescence offers, without doubt, a very broad range of probes for the monitoring of processes related to photosynthesis.

The parameters derived from fluorescence signals can provide information about the structure (PSII antenna size, photosystem integrity and stoichiometry of components of the ETC) and function of PSII and the ETC. It has to be emphasized once more that the utility $\mathrm{Chl} a$ fluorescence is not limited to PSII. Chl $a$ fluorescence measurements can also give insight into the function and content of PSI, cyclic electron flow and excitation transfer to and between the photosystems. Several fluorescence methods (e.g., prompt and delayed Chl $a$ fluorescence), measured using various protocols and instruments, are applied at different temperatures to decipher the functional integrity of the pigment protein complexes in different photosystems. The impact of biotic and abiotic environmental stress on green plants - but also on algae and cyanobacteria — and in many cases their early detection is possible. Fluorescence methods can also be combined with other photosynthetic techniques to help decipher the intricate regulation and complex interactions between a plant's metabolic systems.

Thanks to the efforts of several generations of scientists in the field of photosynthetic research, $\mathrm{Chl} a$ fluorescence is becoming more and more one of the success stories in plant physiology. Over the last decades the number of users of this technique in basic and applied research has grown exponentially. However, if new users lack sufficient basic knowledge of $\mathrm{Chl} a$ fluorescence and of the experimental possibilities of Chl $a$ fluorescence-based techniques, there is a high risk that the users will misinterpret or overinterpret their results, that they will insufficiently take into account the preconditions that have to be met to successfully apply $\mathrm{Chl} a$ fluorescence and that they will not make use of all the possibilities the method offers to them.

In this review we have treated an additional set of questions relevant to contemporary $\mathrm{Chl} a$ fluorescence research. Nevertheless, many details and specific aspects of $\mathrm{Chl} a$ fluorescence are beyond the scope of this review and every reader is urged to consult the primary literature for a fuller treatment of this rapidly moving area of research. Finally, as many of the treated questions imply, much is still unknown, many topics rest on weak foundations, new protocols can be designed, and new instruments developed. There is still considerable potential for chlorophyll fluorescence techniques to be improved and to expand, providing new insights into the fundamental process of photosynthesis.

Acknowledgements The authors would like to thank the following prominent scientists for encouraging us and to prepare this 
manuscript, their important leads to enhance the quality of the text, and fruitful discussions: Govindjee, Suleyman Allakhverdiev, George Papageorgiou, Merope Tsimilli-Michael, Giles Johnson, Helmut Lichtenthaler, Ulrich Schreiber, Jeremy Harbinson, Bernard Genty and João Serôdio.

Open Access This article is distributed under the terms of the Creative Commons Attribution 4.0 International License (http://crea tivecommons.org/licenses/by/4.0/), which permits unrestricted use, distribution, and reproduction in any medium, provided you give appropriate credit to the original author(s) and the source, provide a link to the Creative Commons license, and indicate if changes were made.

\section{References}

Adams WW III, Demmig-Adams B (1992) Operation of the xanthophyll cycle in higher plants in response to diurnal changes in incident sunlight. Plant 186:390-398

Adams WW III, Demmig-Adams B (2004) Chlorophyll fluorescence as a tool to monitor plant response to the environment. In: Papageorgiou GC, Govindjee (eds) Advances in photosynthesis and respiration series chlorophyll fluorescence: a signature of photosynthesis, vol 19. Springer, Dordrecht, pp 583-604

Adams WW III, Demmig-Adams B, Winter K, Schreiber U (1990a) The ratio of variable to maximum chlorophyll fluorescence from photosystem II, measured in leaves at ambient temperature and at $77 \mathrm{~K}$, as an indicator of the photon yield of photosynthesis. Planta 180:166-174

Adams WW III, Winter K, Schreiber U, Schramel P (1990b) Photosynthesis and chlorophyll fluorescence characteristics in relationship to changes in pigment and element composition of leaves of Platanus occidentalis L. during autumnal senescence. Plant Physiol 93:1184-1190

Alfonso M, Montoya G, Cases R, Rodriguez R, Picorel R (1994) Core antenna complexes, CP43 and CP47, of higher plant photosystem II. Spectral properties, pigment stoichiometry, and amino acid composition. Biochemistry 33:10494-10500

Allakhverdiev SI (2011) Recent progress in the studies of structure and function of photosystem II. J Photochem Photobiol B Biol $104: 1-8$

Allakhverdiev SI, Klimov VV, Carpentier R (1994) Variable thermal emission and chlorophyll fluorescence in photosystem II particles. Proc Natl Acad Sci USA 491:281-285

Allakhverdiev SI, Los DA, Mohanty P, Nishiyama Y, Murata N (2007) Glycinebetaine alleviates the inhibitory effect of moderate heat stress on the repair of photosystem II during photoinhibition. Biochim Biophys Acta 1767:1363-1371

Allen JF (1992) Protein phosphorylation in regulation of photosynthesis. Biochim Biophys Acta 1098:275-335

Allen JF, Bennett J, Steinback KE, Arntzen CJ (1981) Chloroplast protein phosphorylation couples platoquinone redox state to distribution of excitation energy between photosystems. Nature 291:21-25

Amesz J, van Gorkom HJ (1978) Delayed fluorescence in photosynthesis. Annu Rev Plant Physiol 29:47-66

Ananyev GM, Dismukes GC (1996) Assembly of the tetra-Mn site of photosynthetic water oxidation by photoactivation: Mn stoichiometry and detection of a new intermediate. Biochemistry 35:4102-4109

Anderson JM, Chow WS, Goodchild DJ (1988) Thylakoid membrane organization in sun/shade acclimation. Aust J Plant Physiol $15: 11-26$
Andrizhiyevskaya EG, Chojnicka A, Bautista JA, Diner BA, van Grondelle R, Dekker JP (2005) Origin of the F685 and F695 fluorescence in photosystem II. Photosynth Res 84:173-180

Anithakumari AM, Nataraja KN, Visser RGF, van der Linden G (2012) Genetic dissection of drought tolerance and recovery potential by quantitative trait locus mapping of a diploid potato population. Mol Breed 30:1413-1429

Antal TK, Krendeleva TE, Rubin AB (2007) Study of photosystem 2 heterogeneity in the sulfur-deficient green alga Chlamydomonas reinhardtii. Photosynth Res 94:13-22

Antal TK, Matorin DN, Ilyash LV, Volgusheva AA, Osipov A, Konyuhow IV, Krendeleva TE, Rubin AB (2009) Probing of photosynthetic reactions in four phytoplanktonic algae with a PEA fluorometer. Photosynth Res 102:67-76

Araus JL, Amaro T, Voltas J, Nakkoul H, Nachit MM (1998) Chlorophyll fluorescence as a selection criterion for grain yield in durum wheat under Mediterranean conditions. Field Crops Res 55:209-223

Argyroudi-Akoyunoglou J (1984) The 77 K fluorescence spectrum of the Photosystem I pigment-protein complex CPIa. FEBS Lett 171:47-53

Arnold WA (1991) Experiments. Photosynth Res 27:73-82

Arnold WA, Thompson J (1956) Delayed light production by bluegreen algae, red algae and purple bacteria. J Gen Physiol 39:311-318

Aro EM, Hundal T, Carlberg I, Andersson B (1990) In vitro studies on light-induced inhibition of PSII and D1-protein degradation at low temperatures. Biochim Biophys Acta 1019:269-275

Aro EM, Virgin I, Andersson B (1993) Photoinhibition of photosystem II. Inactivation protein damage and turnover. Biochim Biophys Acta 1143:113-134

Arsalane W, Parésys G, Duval JC, Wilhelm C, Conrad R, Büchel C (1993) A new fluorometric device to measure the in vivo chlorophyll $a$ fluorescence yield in microalgae and its use as a herbicide monitor. Eur J Phycol 28:247-252

Asada K (1999) The water-water cycle in chloroplasts: scavenging of active oxygens and dissipation of excess photons. Annu Rev Plant Physiol Plant Mol Biol 50:601-639

Ashraf M, Harris PJC (2004) Potential biochemical indicators of salinity tolerance in plants. Plant Sci 166:3-16

Bailey S, Walters RG, Jansson S, Horton P (2001) Acclimation of Arabidopsis thaliana to the light environment: the existence of separate low light and high light responses. Planta 213:794-801

Baker NR (2008) Chlorophyll fluorescence: a probe of photosynthesis in vivo. Annu Rev Plant Biol 59:659-668

Baker NR, Rosenqvist E (2004) Applications of chlorophyll fluorescence can improve crop production strategies: an examination of future possibilities. J Exp Bot 55:1607-1621

Ballottari M, Dall'Osto L, Morosinotto T, Bassi R (2007) Contrasting behavior of higher plant photosystem I and II antenna systems during acclimation. J Biol Chem 282:8947-8958

Barbagallo RP, Oxborough K, Pallett KE, Baker NR (2003) Rapid, noninvasive screening for perturbations of metabolism and plant growth using chlorophyll fluorescence imaging. Plant Physiol 132:485-493

Barber J, Malkin S, Telfer A (1989) The origin of chlorophyll fluorescence in vivo and its quenching by the photosystem II reaction centre. Philos Trans R Soc Lond B 323:227-239

Barra M, Haumann M, Loja P, Krivanek R, Grundmeier A, Dau H (2006) Intermediates in assembly by photoactivation after thermally accelerated disassembly of the manganese complex of photosynthetic water oxidation. Biochemistry 45:14523-14532

Baumann HA, Morrison L, Stengel DB (2009) Metal accumulation and toxicity measured by PAM-chlorophyll fluorescence in 
seven species of marine macroalgae. Ecotoxicol Environ Safe 72:1063-1075

Bauwe H, Hagemann M, Fernie A (2010) Photorespiration: players, partners and origin. Trends Plant Sci 15:330-336

Beck WF, Brudvig GW (1987) Reactions of hydroxylamine with the electron-donor side of photosystem II. Biochemistry 26:8285-8295

Belgio E, Kapitonova E, Chmeliov J, Duffy CDP, Ungerer P, Valkunas L, Ruban AV (2014) Economic photoprotection in photosystem II that retains a complete light-harvesting system with slow energy traps. Nat Commun 5:4433. doi:10.1038/ ncomms 5433

Bell DH, Hipkins MF (1985) Analysis of fluorescence induction curves from pea chloroplasts: photosystem II reaction centre heterogeneity. Biochim Biophys Acta 807:255-262

Bellafiore S, Barneche F, Peltier G, Rochaix J-D (2005) State transitions and light adaptation require chloroplast thylakoid protein kinase STN7. Nature 433:892-895

Belyaeva NE, Schmitt F-J, Paschenko VZ, Riznichenko GY, Rubin $A B$ (2015) Modeling of the redox state dynamics in photosystem II of Chlorella pyrenoidosa Chick cells and leaves of spinach and Arabidopsis thaliana from single flash-induced fluorescence quantum yield changes on the $100 \mathrm{~ns}-10 \mathrm{~s}$ time scale. Photosynth Res 125:123-140

Bennett J (1977) Phosphorylation of chloroplast membrane polypeptides. Nature 269:344-346

Bennett J (1983) Regulation of photosynthesis by reversible phosphorylation of the light-harvesting chlorophyll $a / b$ protein. Biochem J 212:1-13

Bennett J, Shaw EK, Michel H (1988) Cytochrome b6f complex is required for phosphorylation of light-harvesting chlorophyll $\mathrm{a} / \mathrm{b}$ complex II in chloroplast photosynthetic membranes. Eur J Biochem 171:95-100

Bennoun P (2002) The present model for chlororespiration. Photosynth Res 73:273-277

Bennoun P, Li Y-S (1973) New results on the mode of action of 3,(3,4-dichlorophenyl)-1,1-dimethylurea in spinach chloroplasts. Biochim Biophys Acta 292:162-168

Berden-Zrimec M, Drinovec L, Zrimec A (2011) Delayed fluorescence. In: Suggett DJ, Borowitzka M, Prášil O (eds) Chlorophyll $a$ fluorescence in aquatic sciences: methods and applications, developments in applied phycology, vol 4. Springer, The Netherlands, pp 293-309

Berger S, Sinha AK, Roitsch T (2007) Plant physiology meets phytopathology: plant primary metabolism and plant-pathogen interactions. J Exp Bot 58:4019-4026

Bernacchi CJ, Leakey ADB, Heady LE, Morgan PB, Dohleman FG, McGrath JM, Gillespie GM, Wittig VE, Rogers A, Long SP, Ort DR (2006) Hourly and seasonal variation in photosynthesis and stomatal conductance of soybean grown at future $\mathrm{CO}_{2}$ and ozone concentrations for 3 years under fully open-air field conditions. Plant Cell Environ 29:2077-2090

Betterle N, Ballotari M, Zorzan S, de Bianchi S, Cazzaniga S, Dall'Osto L, Morosinotto T, Bassi R (2009) Light-induced dissociation of an antenna hetero-oligomer is needed for nonphotochemical quenching induction. $\mathrm{J}$ Biol Chem 284:15255-15266

Bielczynski LW, Schansker G, Croce R (2016) Effect of light acclimation on the organization of photosystem II super and subcomplexes in Arabidopsis thaliana. Front Plant Sci. doi:10.3389/ fpls.2016.00105

Björkman O, Demmig-Adams B (1995) Regulation of photosynthetic light energy capture, conversion, and dissipation in leaves of higher plants. In: Schulze ED, Caldwell MM (eds) Ecophysiology of photosynthesis. Springer, Berlin, pp 17-47
Blubaugh DJ, Cheniae GM (1990) Kinetics of photoinhibition in hydroxylamine-extracted photosystem II membranes: relevance to photoactivation and site of electron donation. Biochemistry 29:5109-5118

Bock A, Krieger-Liszkay A, Ortiz de Zarate IB, Schönknecht G (2001) $\mathrm{Cl}$-channel inhibitors of the arylaminobenzoate type act as photosystem II herbicides: a functional and structural study. Biochemistry 40:3273-3281

Bode S, Quentmeier CC, Liao P-N, Hafi N, Barros T, Wilk L, Bittner F, Walla PJ (2009) On the regulation of photosynthesis by excitonic interactions between carotenoids and chlorophylls. Proc Natl Acad Sci USA 106:12311-12316

Boekema EJ, Van Roon H, Van Breemen JFL, Dekker JP (1999) Supramolecular organization of photosystem II and its lightharvesting antenna in partially solubilized photosystem II membranes. Eur J Biochem 266:444-452

Bolhar-Nordenkampf HR, Long SP, Baker NR, Öquist G, Schreiber U, Lechner EG (1989) Chlorophyll fluorescence as a probe of the photosynthetic competence of leaves in the field: a review of current Instrumentation. Funct Ecol 3:497-514

Bonaventura C, Myers J (1969) Fluorescence and oxygen evolution from Chlorella pyrenoidosa. Biochim Biophys Acta 189:366-383

Bonfig KB, Schreiber U, Gabler A, Roitsch T, Berger S (2006) Infection with virulent and avirulent $P$. syringae strains differentially affects photosynthesis and sink metabolism in Arabidopsis leaves. Planta 225:1-12

Bouges-Bocquet B (1980) Kinetic models for the electron donors of photosystem II of photosynthesis. Biochim Biophys Acta 594:85-103

Bradbury M, Baker NR (1981) Analysis of the slow phases of the in vivo chlorophyll fluorescence induction curve; changes in the redox state of photosystem II electron acceptors and fluorescence emission from photosystem I and II. Biochim Biophys Acta 635:542-551

Brestič M, Živčák M (2013) PSII fluorescence techniques for measurement of drought and high temperature stress signal in crop plants: protocols and applications. In: Das AB, Rout GR (eds) Molecular stress physiology of plants. Springer, New Dehli, pp 87-131

Brestič M, Cornic G, Fryer MJ, Baker NR (1995) Does photorespiration protect the photosynthetic apparatus in French bean leaves from photoinhibition during drought stress? Planta 196:450-457

Brestič M, Živčák M, Kalaji HM, Allakhverdiev SI, Carpentier R (2012) Photosystem II thermo-stability in situ: environmentally induced acclimation and genotype-specific reactions in Triticum aestivum L. Plant Physiol Biochem 57:93-105

Brody SS, Rabinowitch E (1957) Excitation lifetime of photosynthetic pigments in vitro and in vivo. Science 125:555-563

Brudvig GW, Casey JL, Sauer K (1983) The effect of temperature on the formation and decay of the multiline EPR signal species associated with photosynthetic oxygen evolution. Biochim Biophys Acta 723:366-371

Bukhov NG, Boucher N, Carpentier R (1997) The correlation between the induction kinetics of the photoacoustic signal and chlorophyll fluorescence in barley leaves is governed by changes in the redox state of the photosystem II acceptor side; a study under atmospheric and high $\mathrm{CO}_{2}$ concentrations. Can $\mathrm{J}$ Bot 75:1399-1406

Bukhov N, Egorova E, Krendeleva T, Rubin A, Wiese C, Heber U (2001) Relaxation of variable chlorophyll fluorescence after illumination of dark-adapted barley leaves as influenced by the redox states of electron carriers. Photosynth Res 70:155-166

Buschmann C, Koscányi L (1989) Light-induced heat production correlated with chlorophyll fluorescence and its quenching. Photosynth Res 21:129-136 
Bussotti F (2004) Assessment of stress conditions in Quercus ilex L. leaves by O-J-I-P chlorophyll $a$ fluorescence analysis. Plant Biosystems 13:101-109

Bussotti F, Agati G, Desotgiu R, Matteini P, Tani C (2005) Ozone foliar symptoms in woody plants assessed with ultrastructural and fluorescence analysis. New Phytol 166:941-955

Bussotti F, Desotgiu R, Cascio C, Pollastrini M, Gravano E, Gerosa G, Marzuoli R, Nali C, Lorenzini G, Salvatori E, Manes F, Schaub M, Strasser RJ (2011a) Ozone stress in woody plants assessed with chlorophyll $a$ fluorescence. A critical reassessment of existing data. Environ Exp Bot 73:19-30

Bussotti F, Pollastrini M, Cascio C, Desotgiu R, Gerosa G, Marzuoli R, Nali C, Lorenzini G, Pellegrini E, Carucci MG, Salvatori E, Fusaro L, Piccotto M, Malaspina P, Manfredi A, Roccotello E, Toscano S, Gottardini E, Cristofori A, Fini A, Weber D, Baldassarre V, Barbanti L, Monti A, Strasser RJ (2011b) Conclusive remarks. Reliability and comparability of chlorophyll fluorescence data from several field teams. Environ Exp Bot 73:116-119

Butler WL (1978) Energy distribution in the photochemical apparatus of photosynthesis. Annu Rev Plant Physiol 29:345-378

Byrdin M, Rimke I, Schlodder E, Stehlik D, Roelofs TA (2000) Decay kinetics and quantum yields of fluorescence in photosystem I from Synechococcus elongatus with P700 in the reduced and oxidized state: Are the kinetics of excited state decay traplimited or transfer-limited? Biophys J 79:992-1007

Caffarri S, Croce R, Cattivelli L, Bassi R (2004) A look within LHCII: differential analysis of the Lhcb1-3 complexes building the major trimeric antenna complex of higher-plant photosynthesis. Biochemistry 43:9467-9476

Calatayud A, Ramirez JW, Iglesias DJ, Barreno E (2002) Effects of ozone on photosynthetic $\mathrm{CO}_{2}$ exchange, chlorophyll a fluorescence and antioxidant systems in lettuce leaves. Physiol Plant 116:308-316

Cascio C, Schaub M, Novak K, Desotgiu R, Bussotti F, Strasser RJ (2010) Foliar responses to ozone of Fagus sylvatica L. seedlings grown in shaded and in full sunlight conditions. Environ Exp Bot 68:188-197

Cazzaniga S, Dall'Osto L, Kong S-G, Wada M, Bassi R (2013) Interaction between avoidance of photon absorption, excess energy dissipation and zeaxanthin synthesis against photooxidative stress in Arabidopsis. Plant J 76:568-579

Ceppi MG, Oukarroum A, Çiçek N, Strasser RJ, Schansker G (2012) The IP amplitude of the fluorescence rise OJIP is sensitive to changes in the photosystem I content of leaves: a study on plants exposed to magnesium and sulfate deficiencies, drought stress and salt stress. Physiol Plant 144:277-288

Chaudhary N, Singh S, Agrawal SB, Agrawal M (2013) Assessment of six Indian cultivars of mung bean against ozone by using foliar injury index and changes in carbon assimilation, gas exchange, chlorophyll fluorescence and photosynthetic pigments. Environ Monit Assess 185:7793-7807

Chen J, Kell A, Acharya K, Kupitz C, Fromme P, Jankowiak R (2015) Critical assessment of the emission spectra of various photosystem II core complexes. Photosynth Res 124:253-265

Cheng L, Fuchigami LH, Breen PJ (2000) Light absorption and partitioning in relation to nitrogen content 'Fuji' apple leaves. J Am Soc Hortic Sci 125:581-587

Choi CJ, Berges JA, Young EB (2012) Rapid effects of diverse toxic water pollutants on chlorophyll $a$ fluorescence: variable responses among freshwater microalgae. Water Res 46:2615-2626

Chow WS, Aro EM (2005) Photoinactivation and mechanisms of recovery. In: Wydrzynski T, Satoh K (eds) Photosystem II: the light-driven water: plastoquinone oxidoreductase, advances in photosynthesis and respiration, vol 22. Springer, Dordrecht, pp 627-648
Chow WS, Fan DY, Oguchi R, Jia H, Losciale P, Youn-Il P, He J, Öquist G, Shen YG, Anderson JM (2012) Quantifying and monitoring functional photosystem II and the stoichiometry of the two photosystems in leaf segments: approaches and approximations. Photosynth Res 113:63-74

Christensen MG, Teicher HB, Streibig JC (2003) Linking fluorescence induction curve and biomass in herbicide screening. Pest Manag Sci 59:1303-1310

Codrea CM, Aittokallio T, Keränen M, Tyystjärvi E, Nevalainen OS (2003) Feature learning with a genetic algorithm for fluorescence fingerprinting of plant species. Pattern Recognit Lett 24:2663-2673

Conjeaud H, Mathis P (1980) The effect of $\mathrm{pH}$ on the reduction kinetics of P-680 in tris-treated chloroplasts. Biochim Biophys Acta 590:353-359

Conrad R, Büchel C, Wilhelm C, Arsalane W, Berkaloff C, Duval JC (1993) Changes in yield of in-vivo fluorescence of chlorophyll a as a tool for selective herbicide monitoring. J Appl Phycol 5:505-516

Cornic G, Massacci A (1996) Leaf photosynthesis under drought stress. In: Baker NR (ed) Photosynthesis and the environment. Kluwer Academic Publisher, Dordrecht, pp 347-366

Cornic G, Fresneau C (2002) Photosynthetic carbon reduction and carbon oxidation cycles are the main electron sinks for photosystems II during a mild drought. Ann Bot 89:887-894

Correia MJ, Chaves MMC, Pereira JS (1990) Afternoon depression in photosynthesis in grapevine leaves-evidence for a high light stress effect. J Exp Bot 41:417-426

Cotrozzi L, Remorini D, Pellegrini E, Landi M, Massai R, Nali C, Guidi L, Lorenzini G (2016) Variations in physiological and biochemical traits of oak seedlings grown under drought and ozone stress. Physiol Plant 157:69-84

Croce R, Zucchelli G, Garlaschi FM, Bassi R, Jennings RC (1997) Excited state equilibration in the photosystem I-light-harvesting I complex: P700 is almost isoenergetic with its antenna. Biochemistry 35:8572-8579

Cser K, Vass I (2007) Radiative and non-radiative charge recombination pathways in photosystem II studied by thermoluminescence and chlorophyll fluorescence in the cyanobacterium Synechocystis 6308. Biochim Biophys Acta 1767:233-243

Czyczyło-Mysza I, Tyrka M, Marcińska Skrzypek E, Karbarz M, Dziurka M, Hura T, Dziurka K, Quarrie SA (2013) Quantitative trait loci for leaf chlorophyll fluorescence parameters, chlorophyll and carotenoid contents in relation to biomass and yield in bread wheat and their chromosome deletion bin assignments. Mol Breed 32:189-210

D’Haene SE, Sobotka R, Bučinská L, Dekker JP, Komenda J (2015) Interaction of the PsbH subunit with a chlorophyll bound to histidine 114 of $\mathrm{CP} 47$ is responsible for the red $77 \mathrm{~K}$ fluorescence of Photosystem II. Biochim Biophys Acta 1847:1327-1334

Dang NC, Zazubovich V, Reppert M, Neupane B, Picorel R, Seibert M, Jankowiak R (2008) The CP43 proximal antenna complex of higher plant photosystem II revisited: modeling and hole burning study. J Phys Chem B 112:9921-9933

Dau H (1994) Molecular mechanisms and quantitative models of variable Photosystem II fluorescence. Photochem Photobiol 60:1-23

Dau H, Sauer K (1992) Electric field effect on the picosecond fluorescence of photosystem II and its relation to the energetics and kinetics of primary charge separation. Biochim Biophys Acta 1102:91-106

Dau H, Zaharieva I, Haumann M (2012) Recent developments in research on water oxidation by photosystem II. Curr Opin Chem Biol 16:3-10

de Wijn R, van Gorkom HJ (2001) Kinetics of electron transfer from $\mathrm{Q}_{\mathrm{A}}$ to $\mathrm{Q}_{\mathrm{B}}$ in photosystem II. Biochemistry 40:11912-11922 
de Wijn R, van Gorkom HJ (2002) The rate of charge recombination in photosystem II. Biochim Biophys Acta 1553:302-308

Debus RJ (1992) The manganese and calcium ions of photosynthetic oxygen evolution. Biochim Biophys Acta 1102:269-352

Degl'Innocenti E, Guidi L, Soldatini GF (2002) Characterization of the photosynthetic response of tobacco leaves to ozone: $\mathrm{CO}_{2}$ assimilation and chlorophyll fluorescence. J Plant Physiol 159:845-853

Dell'Aquila AR, Van der Schoor R, Jalink H (2002) Application of chlorophyll fluorescence in sorting controlled deteriorated white cabbage (Brassica oleracea L.) seeds. Seed Sci Technol 30:689-695

Demeter S, Zs Rósza, Vass I, Sallai A (1985) Thermoluminescence study of charge recombination in photosystem II at low temperatures. I. Characterization of the $\mathrm{Zv}$ and A thermoluminescence bands. Biochim Biophys Acta 809:369-378

Demeter S, Goussias Ch, Bernát G, Kovács L, Petrouleas V (1993) Participation of the $\mathrm{g}=1.9$ and $\mathrm{g}=1.82 \mathrm{EPR}$ forms of the semiquinone-iron complex, $\mathrm{Q}_{\mathrm{A}}^{-} \mathrm{Fe}^{2+}$ of photosystem II in the generation of the $\mathrm{Q}$ and $\mathrm{C}$ thermoluminescence bands, respectively. FEBS Lett 336:352-356

Demetriou G, Neonaki C, Navakoudis E, Kotzabasis K (2007) Salt stress impact on the molecular structure and function of the photosynthetic apparatus - the protective role of polyamines. Biochim Biophys Acta 1767:272-280

Demmig B, Winter K (1988) Characterisation of three components of non-photochemical fluorescence quenching and their response to photoinhibition. Aust J Plant Physiol 15:163-177

Demmig-Adams B, Adams WW III, Barker DH, Logan BA, Bowling DR, Verhoeven AS (1996) Using chlorophyll fluorescence to assess the fraction of absorbed light allocated to thermal dissipation of excess excitation. Physiol Plant 98:253-264

Demmig-Adams B, Cohu CM, Muller O, Adams WW III (2012) Modulation of photosynthetic energy conversion efficiency in nature: from seconds to seasons. Photosynth Res 113:75-88

Demmig-Adams B, Garab G, Adams WW III, Govindjee (2014) Nonphotochemical quenching and energy dissipation in plants, algae and cyanobacteria. Advances in photosynthesis and respiration, vol 40. Springer, Dordrecht

Depège N, Bellafiore S, Rochaix J-D (2003) Role of chloroplast protein kinase Stt7 in LHCII phosphorylation and state transition in Chlamydomonas. Science 299:1572-1575

Desotgiu R, Cascio C, Pollastrini M, Gerosa G, Marzuoli R, Bussotti F (2012a) Short and long term photosynthetic adjustments in sun and shade leaves of Fagus sylvatica L., investigated by fluorescence transient (FT) analysis. Plant Biosystems 146:206-216

Desotgiu R, Pollastrini M, Cascio C, Gerosa G, Marzuoli R, Bussotti F (2012b) Chlorophyll $a$ fluorescence analysis along a vertical gradient of the crown in a poplar (Oxford clone) subjected to ozone and water stress. Tree Physiol 32:976-986

Desotgiu R, Pollastrini M, Cascio C, Gerosa G, Marzuoli R, Bussotti F (2013) Responses to ozone on Populus "Oxford" clone in an open top chamber experiment assessed before sunrise and in full sunlight. Photosynthetica 51:267-280

DeVault D, Govindjee, Arnold W (1983) Energetics of photosynthetic glow peaks. Proc Natl Acad Sci USA 80:983-987

Dorigo U, Leboulanger C (2001) A pulse-amplitude modulated fluorescence-based method for assessing the effects of photosystem II herbicides on freshwater periphyton. J Appl Phycol 13:509-515

Doschek WW, Kok B (1972) Photon trapping in photosystem II of photosynthesis; the fluorescence rise curve in the presence of 3-(3,4-dichlorophenyl)-1,1-dimethylurea. Biophys J 12:832-838

Ducruet JM (1999) Relation between the heat-induced increase of F0 fluorescence and a shift in the electronic equilibrium at the acceptor side of photosystem 2. Photosynthetica 37:335-338
Ducruet J-M, Miranda T (1992) Graphical and numerical analysis of thermoluminescence and fluorescence F0 emission in photosynthetic material. Photosynth Res 33:15-27

Ducruet JM, Vavilin D (1999) Chlorophyll high-temperature thermoluminescence emission as an indicator of oxidative stress: perturbating effects of oxygen and leaf water content. Free Radical Res 31:S187-S192

Ducruet JM, Vass I (2009) Thermoluminescence: experimental. Photosynth Res 101:195-204

Ducruet J-M, Peeva V, Havaux M (2007) Chlorophyll thermofluorescence and thermoluminescence as complementary tools for the study of temperature stress in plants. Photosynth Res 93:159-171

Duysens LNM, Sweers HE (1963) Mechanisms of two photochemical reactions in algae as studied by means of fluorescence. In: Mahlis L (ed) Studies on microalgae and photosynthetic bacteria, special issue of plant cell physiol. Japanese Society of Plant Physiologists, University of Tokyo Press, Tokyo, pp 353-372

Duysens LNM, Amesz J, Kamp BM (1961) Two photochemical systems in photosynthesis. Nature 190:510-511

Ebenhöh O, Fucile G, Finazzi G, Rochaix J-D, GoldschmidtClermont M (2014) Short-term acclimation of the photosynthetic electron transfer chain to changing light: a mathematical model. Philos Trans R Soc B 369:20130223

Edwards GE, Baker NR (1993) Can $\mathrm{CO}_{2}$ assimilation in maize leaves be predicted accurately from chlorophyll fluorescence analysis? Photosynth Res 37:89-102

Edwards G, Walker D (1983) C3, C4: mechanisms, and cellular and environmental regulation, of photosynthesis. University of California Press, California

Ehleringer J, Pearcy RW (1983) Variation in quantum yield for $\mathrm{CO}_{2}$ uptake among C3 and C4 plants. Plant Physiol 73:555-559

Eichelmann H, Talts E, Oja V, Padu E, Laisk A (2009) Rubisco in planta $\mathrm{k}_{\mathrm{cat}}$ is regulated in balance with photosynthetic electron transport. J Exp Bot 60:4077-4088

Emerson R, Lewis CM (1943) The dependence of the quantum yield of Chlorella photosynthesis on wavelength of light. Am J Bot 30:165-178

Etienne A-L, Ducruet J-M, Ajlani G, Vernotte C (1990) Comparative studies on electron transfer in photosystem II of herbicideresistant mutants from different organisms. Biochim Biophys Acta 1015:435-440

Eyles A, Smith D, Pinkard EA, Smith I, Corkrey R, Elms S, Beadle C, Mohammed C (2011) Photosynthetic responses of field-grown Pinus radiata trees to artificial and aphid-induced defoliation. Tree Physiol 31:592-603

Fai PB, Grant A, Reid B (2007) Chlorophyll $a$ fluorescence as a biomarker for rapid toxicity assessment. Environ Toxicol Chem 26:1520-1531

Faller P, Pascal A, Rutherford AW (2001) $\beta$-Carotene redox reactions in photosystem II: electron transfer pathway. Biochemistry 40:6431-6440

Falster DS, Westoby M (2003) Leaf size and angle vary widely across species: what consequences for light interception? New Phytol 158:509-525

Felle HH, Hanstein S, Steinmeyer R, Hedrich R (2000) Dynamics of ionic activities in the apoplast of the sub-stomatal cavity of intact Vicia faba leaves during stomatal closure evoked by ABA and darkness. Plant J 24:297-304

Feller U, Crafts-Brandner SJ, Salvucci E (1998) Moderately high temperatures inhibit ribulose-1,5-bisphosphate carboxylase/oxygenase activase mediated activation of Rubisco. Plant Physiol 116:539-546

Ferroni L, Baldisserotto C, Giovanardi M, Pantaleoni L, Morosinotto T, Pancaldi S (2011) Revised assignment of room-temperature chlorophyll fluorescence emission bands in single living cells of 
Chlamydomonas reinhardtii. J Bioenergy Biomembr 43:163-173

Ferroni L, Pantaleoni L, Baldisserotto C, Aro EM, Pancaldi S (2013) Low photosynthetic activity is linked to changes in the organization of photosystem II in the fruit of Arum italicum. Plant Physiol Biochem 63:140-150

Ferroni L, Angeleri M, Pantaleoni L, Pagliano C, Longoni P, Marsano F, Aro EM, Suorsa M, Baldisserotto C, Giovanardi M, Cella R, Pancaldi S (2014) Light-dependent reversible phosphorylation of the minor photosystem II antenna Lhcb6 (CP24) occurs in lycophytes. Plant J 77:893-905

Ferroni L, Suorsa M, Aro EM, Baldisserotto C, Pancaldi S (2016) Light acclimation in the lycophyte Selaginella martensii depends on changes in the amount of photosystems and on the flexibility of LHCII antenna association to both photosystems. New Phytol 211:554-568

Flexas J, Medrano H (2002) Energy dissipation in C3 plants under drought. Funct Plant Biol 29:1209-1215

Flexas J, Escalona JM, Medrano H (1999) Water stress induces different levels of photosynthesis and electron transport rate regulation in grapevines. Plant Cell Environ 22:39-48

Flexas J, Escalona JM, Evain S, Gulías J, Moya I, Osmond CB, Medrano H (2002) Steady-state chlorophyll fluorescence (Fs) measurements as a tool to follow variations of net $\mathrm{CO}_{2}$ assimilation and stomatal conductance during water-stress in $\mathrm{C}_{3}$ plants. Physiol Plant 114:231-240

Flood PJ, Harbinson J, Aarts MGM (2011) Natural genetic variation in plant photosynthesis. Trends Plant Sci 16:327-335

Foyer CH, Noctor G (2009) Redox regulation in photosynthetic organisms: signaling, acclimation, and practical implications. Antioxid Redox Signal 11:861-905

Fracheboud Y, Leipner J (2003) The application of chlorophyll fluorescence to study light, temperature, and drought stress. In: DeEll JR, Toivonen PMA (eds) Practical applications of chlorophyll fluorescence in plant biology. Kluwer Academic Publishers, Dordrecht, pp 125-150

Fracheboud Y, Ribaut J-M, Vargas M, Messmer R, Stamp P (2002) Identification of quantitative trait loci for cold-tolerance of photosynthesis in maize (Zea mays L.). J Exp Bot 53:1967-1977

Fracheboud Y, Jompuk C, Ribaut JM, Stamp P, Leipner J (2004) Genetic analysis of cold-tolerance of photosynthesis in maize. Plant Mol Biol 56:241-253

Franck F, Juneau P, Popovich R (2002) Resolution of the photosystem I and photosystem II contributions to chlorophyll fluorescence of intact laves at room temperature. Biochim Biophys Acta 1556:239-246

Franco AC, Herzog B, Hübner C, de Mattos EA, Scarano FR, Ball E, Lüttge U (1999) Diurnal changes in chlorophyll a fluorescence, $\mathrm{CO}_{2}$-exchange and organic acid decarboxylation in the tropical CAM tree Clusia hilariana. Tree Physiol 19:635-644

Froux F, Ducrey M, Epron D, Dreyer E (2004) Seasonal variations and acclimation potential of the thermostability of photochemistry in four Mediterranean conifers. Ann For Sci 61:235-241

Fuerst EP, Nakatani HY, Dodge AD, Penner D, Arntzen CJ (1985) Paraquat resistance in conyza. Plant Physiol 77:984-989

Fufezan C, Rutherford AW, Krieger-Liszkay A (2002) Singlet oxygen production in herbicide-treated photosystem II. FEBS Lett 532:407-410

Gaevsky NA, Morgun VN (1993) Use of variable and delayed chlorophyll fluorescence for the study of plant photosynthesis. Fiziol Rast 40:136-145 [in Russian]

García-Plazaola JI, Faria T, Abaía J, Abadía A, Chaves MM, Pereira JS (1997) Seasonal changes in xanthophyll composition and photosynthesis of cork oak (Quercus suber L.) leaves under mediterranean climate. J Exp Bot 48:1667-1674

Garnier J, Maroc J, Guyon D (1986) Low-temperature fluorescence emission spectra and chlorophyll-protein complexes in mutants of Chlamydomonas reinhardtii: evidence for a new chlorophyll$a$-protein complex related to photosystem I. Biochim Biophys Acta 85:395-406

Genty B, Harbinson J (2004) The regulation of light utilization for photosynthetic electron transport. In: Baker NR (ed) Photosynthesis and environment. Advances in photosynthesis and respiration, vol 5. Kluwer Academic Publisher, Dordrecht, pp 67-99

Genty B, Briantais J-M, Baker NR (1989) The relationship between the quantum yield of photosynthetic electron transport and quenching of chlorophyll fluorescence. Biochim Biophys Acta 990:87-92

Genty B, Harbinson J, Baker NR (1990a) Relative quantum efficiencies of the two photosystems of leaves in photorespiratory and non-photorespiratory conditions. Plant Physiol Biochem 28:1-10

Genty B, Wonders J, Baker NR (1990b) Non-photochemical quenching of $\mathrm{F}_{O}$ in leaves is emission wavelength dependent. Consequences for quenching analysis and its interpretation. Photosynth Res 26:133-139

Gibasiewicz K, Croce R, Morosinotto T, Ihalainen JA, van Stokkum IHM, Dekker JP, Bassi R, van Grondelle R (2005) Excitation energy transfer pathways in Lhca4. Biophys J 88:1959-1969

Gilmore AM, Shinkarev VP, Hazlett TL, Govindjee (1998) Quantitative analysis of the effects of intrathylakoid $\mathrm{pH}$ and xanthophyll cycle pigments on chlorophyll $a$ fluorescence lifetime distributions and intensity in thylakoids. Biochemistry 37:13582-13593

Gitelson AA, Buschmann C, Lichtenthaler HK (1998) Leaf chlorophyll fluorescence corrected for re-absorption by means of absorption and reflectance measurements. J Plant Physiol 152:283-296

Goldschmidt-Clermont M, Bassi R (2015) Sharing light between two photosystems: mechanism of state transitions. Curr Opin Plant Biol 25:71-78

Goltsev V, Yordanov I (1997) Mathematical model of prompt and delayed chlorophyll fluorescence induction kinetics. Photosynthetica 33:571-586

Goltsev V, Zaharieva I, Lambrev P, Yordanov I, Strasser R (2003) Simultaneous analysis of prompt and delayed chlorophyll $a$ fluorescence in leaves during the induction period of dark to light adaptation. J Theor Biol 225:171-183

Goltsev V, Chernev P, Zaharieva I, Lambrev P, Strasser RJ (2005) Kinetics of delayed chlorophyll $a$ fluorescence registered in milliseconds time range. Photosynth Res 84:209-215

Goltsev V, Zaharieva I, Chernev P, Strasser RJ (2009) Delayed fluorescence in photosynthesis. Photosynth Res 101:217-232

Goltsev V, Zaharieva I, Chernev P, Kouzmanova M, Kalaji H, Yordanov I, Krasteva V, Alexandrov V, Stefanov D, Allakhverdiev S, Strasser RJ (2012) Drought-induced modifications of photosynthetic electron transport in intact leaves: analysis and use of neural networks as a tool for a rapid non-invasive estimation. Biochim Biophys Acta 1817:1490-1498

Gorbe E, Calatayud A (2012) Applications of chlorophyll fluorescence imaging technique in horticultural research: a review. Sci Hortic 138:24-35

Gorbunov MY, Kolber ZS, Falkowski PG (1999) Measuring photosynthetic parameters in individual algal cells by fast repetition rate fluorometry. Photosynth Res 62:141-153

Govindjee, Jursinic PA (1979) Photosynthesis and fast changes in light emission by green plants. In: Smith KC (ed) Photochemical and photobiological reviews, vol 4. Plenum Press, New York, pp 125-205

Govindjee (1995) Sixty years since Kautsky: chlorophyll $a$ fluorescence. Aust J Plant Physiol 22:131-160

Govindjee (1999) On the requirement of minimum number of four versus eight quanta of light for the evolution of one molecule of 
oxygen in photosynthesis: a historical note. Photosynth Res 59:249-254

Govindjee, Papageorgiou G (1971) Chlorophyll fluorescence and photosynthesis: fluorescence transients. Photophysiol 6:1-50

Govindjee R, Rabinowitch E, Govindjee (1968) Maximum quantum yield and action spectrum of photosynthesis and fluorescence in Chlorella. Biochim Biophys Acta 162:539-544

Govindjee, Amesz J, Fork DJ (1986) Light emission by plants and bacteria. Academic Press, New York

Grabolle M, Dau H (2005) Energetics of primary and secondary electron transfer in photosystem II membrane particles of spinach revisited on basis of recombination-fluorescence measurements. Biochim Biophys Acta 1708:209-218

Grabolle M, Dau H (2007) Efficiency and role of loss processes in light-driven water oxidation by PSII. Physiol Plant 131:50-63

Greaves JA, Wilson JM (1987) Chlorophyll fluorescence analysisan aid to plant breeders. Biologist 34:209-214

Grieco M, Tikkanen M, Paakkarinen V, Kangasjärvi S, Aro E-M (2012) Steady-state phosphorylation of light-harvesting complex II proteins preserves photosystem I under fluctuating white light. Plant Physiol 160:1896-1910

Grieco M, Suorsa M, Jajoo A, Tikkanen M, Aro EM (2015) Lightharvesting II antenna trimers connect energetically the entire photosynthetic machinery-including both photosystems II and I. Biochim Biophys Acta 1847:607-619

Groot ML, Frese RN, de Weerd FL, Bromek K, Petterson A, Peterman EJG, van Stokkum IHM, van Grondelle R, Dekker JP (1999) Spectroscopic properties of the CP43 core antenna protein of photosystem II. Biophys J 77:3328-3340

Grouneva I, Jakob T, Wilhelm C, Goss R (2009) The regulation of xanthophyll cycle activity and of non-photochemical fluorescence quenching by two alternative electron flows in the diatoms Phaeodactylum tricornutum and Cyclotella meneghiniana. Biochim Biophys Acta 1787:929-938

Guidi L, Calatayud A (2014) Non-invasive tools to estimate stressinduced changes in photosynthetic performance in plants inhabiting Mediterranean areas. Environ Exp Bot 103:42-52

Guidi L, Nali C, Ciompi S, Lorenzini G, Soldatini GF (1997) The use of chlorophyll fluorescence and leaf gas exchange as methods for studying the different responses to ozone of two bean cultivars. J Exp Bot 48:173-179

Guidi L, Tonini M, Soldatini GF (2000) Effects of high light and ozone fumigation on photosynthesis in Phaseolus vulgaris. Plant Physiol Biochem 38:717-725

Guidi L, Degl'Innocenti E, Giordano C, Biricolti S, Tattini M (2010) Ozone tolerance in Phaseolus vulgaris depends on more than one mechanism. Environ Pollut 158:3164-3171

Guidi L, Degl'Innocenti E, Remorini D, Biricolti S, Fini A, Ferrini F, Nicese FP, Tattini M (2011) The impact of UV-radiation on the physiology and biochemistry of Ligustrum vulgare exposed to different visible-light irradiance. Environ Exp Bot 70:88-95

Guo P, Baum M, Varshney RK, Graner A, Grando S, Ceccarelli S (2008) QTLs for chlorophyll and chlorophyll fluorescence parameters in barley under post-flowering drought. Euphytica 163:203-214

Hall JL (2002) Cellular mechanisms for heavy metal detoxification and tolerance. J Exp Bot 53:1-11

Harbinson J, Hedley CL (1993) Changes in P-700 oxidation during the early stages of the induction of photosynthesis. Plant Physiol 103:649-660

Harbinson J, Rosenqvist E (2003) An introduction to chlorophyll fluorescence. In: DeEll J, Toivonen PMA (eds) Practical applications of chlorophyll fluorescence in plant biology. Kluwer Academic Publishers, Dordrecht, pp 1-30

Harbinson J, Genty B, Foyer CH (1990) Relationship between photosynthetic electron transport and stromal enzyme activity in pea leaves toward an understanding of the nature of photosynthetic control. Plant Physiol 94:545-553

Haynes D, Ralph P, Prange J, Dennison W (2000) The impact of the herbicide diuron on photosynthesis in three species of tropical seagrass. Mar Pollut Bull 41:288-293

Heber U, Walker D (1992) Concerning a dual function of coupled cyclic electron transport in leaves. Plant Physiol 100:1621-1626

Heber U, Neimanis S, Dietz K-J (1988) Fractional control of photosynthesis by the $\mathrm{Q}_{\mathrm{B}}$ protein, the cytochrome $\mathrm{f} / \mathrm{b}_{6}$ complex and other components of the photosynthetic apparatus. Planta 173:267-274

Henriques FS (2009) Leaf chlorophyll fluorescence: background and fundamentals for plant biologists. Bot Rev 75:249-270

Hermans C, Smeyers M, Maldonado-Rodriguez R, Eyletters M, Strasser RJ, Delhaye P-J (2003) Quality assessment of urban trees: a comparative study of physiological characterisation, airborne imaging and on site fluorescence by the OJIP-test. J Plant Physiol 160:81-90

Hess FD (2000) Light-dependent herbicides: an overview. Weed Sci 48:160-170

Hideg E, Schreiber U (2007) Parallel assessment of ROS formation and photosynthesis in leaves by fluorescence imaging. Photosynth Res 92:103-108

Hideg E, Vass I (1993) The $75^{\circ} \mathrm{C}$ thermoluminescence band of green tissues: chemiluminescence from membrane-chlorophyll interaction. Photochem Photobiol 58:280-283

Hideg E, Sass L, Barbato R, Vass I (1993) Interaction of photosynthetic oxygen evolution by UV-B irradiation: a thermoluminescence study. Photosynth Res 38:455-462

Hogewoning SW, Harbinson J (2007) Insights on the development, kinetics, and variation of photoinhibition using chlorophyll fluorescence imaging of a chilled, variegated leaf. J Exp Bot $58: 453-463$

Hogewoning SW, Wientjes E, Douwstra P, Trouwborst G, van Ieperen W, Croce R, Harbinson J (2012) Photosynthetic quantum yield dynamics: from photosystems to leaves. Plant Cell 24:1921-1935

Holzwarth AR, Miloslavina Y, Nilkens M, Jahns P (2009) Identification of two quenching sites active in the regulation of photosynthetic light-harvesting studied by time-resolved fluorescence. Chem Phys Lett 483:262-267

Horton P, Hague A (1988) Studies on the induction of chlorophyll fluorescence in isolated barley protoplasts: IV. Resolution of nonphotochemical quenching. Biochim Biophys Acta 932:107-115

Hund A, Frascaroli E, Leipner J, Jompuk C, Stamp P, Fracheboud Y (2005) Cold tolerance of the photosynthetic apparatus: pleiotropic relationship between photosynthetic performance and specific leaf area of maize seedlings. Mol Breed 16:321-331

Ilík P, Schansker G, Kotabová E, Váczi P, Strasser RJ, Bartak M (2006) A dip in the chlorophyll fluorescence induction at 0.2-2 s in Trebouxia-possessing lichens reflects a fast reoxidation of photosystem I. A comparison with higher plants. Biochim Biophys Acta 1757:12-20

Ioannidis N, Schansker G, Barynin VV, Petrouleas V (2000) Interaction of nitric oxide with the oxygen evolving complex of photosystem II and manganese catalase: a comparative study. J Bioinorg Chem 5:354-363

Ishida A, Toma T, Marjenah (1999) Leaf gas exchange and chlorophyll fluorescence in relation to leaf angle, azimuth, and canopy position in the tropical pioneer tree, Macaranga conifer. Tree Physiol 9:117-124

Itoh S, Murata N (1973) Correlation between delayed light emission and fluorescence of chlorophyll $a$ in system II particles derived from spinach chloroplasts. Photochem Photobiol 18:209-218

Itoh S, Katoh S, Takamiya A (1971) Studies on the delayed light emission in spinach chloroplasts; II. Participation of primary 
electron donor and acceptor of photoreaction II in producing the delayed light emission. Biochim Biophys Acta 245:121-128

Iwai M, Takahashi Y, Minagawa J (2008) Molecular remodeling of photosystem II during state transitions in Chlamydomonas reinhardtii. Plant Cell 20:2177-2189

Jahns P, Holzwarth AR (2012) The role of the xanthophyll cycle and of lutein in photoprotection of photosystem II. Biochim Biophys Acta 1817:182-193

Jalink H, Van der Schoor R, Frandas A, Van Pijlen JG (1998) Chlorophyll fluorescence of Brassica oleracea seeds as a nondestructive marker for seed maturity and seed performance. Seed Sci Res 8:437-443

Jifon JL, Syvertsen JP (2003) Moderate shade can increase net gas exchange and reduce photoinhibition in citrus leaves. Tree Physiol 23:119-127

Johnson GN (2011) Physiology of PSI cyclic electron transport in higher plants. Biochim Biophys Acta 1807:384-389

Johnson MP, Goral TK, Duffy CD, Brain AP, Mullineaux CW, Ruban AV (2011) Photoprotective energy dissipation involves the reorganization of photosystem II light-harvesting complexes in the grana membranes of spinach chloroplasts. Plant Cell 23:1468-1479

Joliot A, Joliot P (1964) Étude cinétique de la reaction photochimique libérant l'oxygène au cours de la photosynthèse. C R Acad Sci Paris 258:4622-4625

Joliot P, Joliot A (2002) Cyclic electron transfer in plant leaf. Proc Natl Acad Sci USA 99:10209-10214

Joliot P, Joliot A, Bouges B, Barbieri G (1971) Studies of system II photocenters by comparative measurements of luminescence, fluorescence, and oxygen emission. Photochem Photobiol 14:287-305

Judy BM, Lower WR, Ireland FA, Krause GF (1991) A seedling chlorophyll fluorescence toxicity assay. In: Gorsuch JW, Lower WR, Lewis MA, Wang W (eds) Plants for toxicity assessment. ASTM, Philadelphia, pp 146-158

Juneau P, Dewez D, Matsui S, Kim SG, Popovic R (2001) Evaluation of different algal species sensitivity to mercury and metolachlor by PAM-fluorometry. Chemosphere 45:589-598

Jung H-S, Niyogi KK (2009) Quantitative genetic analysis of thermal dissipation in Arabidopsis. Plant Physiol 150:977-986

Jursinic P (1986) Delayed fluorescence: current concepts and status. In: Amesz J, Fork DJ, Govindjee (eds) Light emission by plants and bacteria. Academic Press, New York, pp 291-328

Jursinic P, Govindjee (1982) Effects of hydroxylamine and silicomolybdate on the decay in delayed light emission in the 6-100 $\mu$ s range after a single 10 ns flash in pea thylakoids. Photosynth Res 3:161-177

Kalaji MH, Guo P (2008) Chlorophyll fluorescence: A useful tool in barley plant breeding programs. In: Sánchez A, Gutierrez SJ (eds) Photochemistry research progress. Nova Science Publishers, New York, pp 439-463

Kalaji MH, Łoboda T (2007) Photosystem II of barley seedlings under cadmium and lead stress. Plant Soil Environ 53:511-516

Kalaji MH, Pietkiewicz S (1993) Salinity effects on plant growth and other physiological processes. Acta Physiol Plant 15:89-124

Kalaji MH, Pietkiewicz S (2004) Some physiological indices to be exploited as a crucial tool in plant breeding. Plant Breed Seeds Sci 49:19-39

Kalaji MH, Bosa K, Kościelniak J, Hossain Z (2011a) Chlorophyll $a$ fluorescence-a useful tool for the early detection of temperature stress in spring barley (Hordeum vulgare L.). OMICS 15:925-934

Kalaji MH, Govindjee, Bosa K, Kościelniak J, Żuk-Gołaszewska K (2011b) Effects of salt stress on photosystem II efficiency and $\mathrm{CO}_{2}$ assimilation of two Syrian barley landraces. Environ Exper Bot 73:64-72
Kalaji MH, Carpentier R, Allakhverdiev SI, Bosa K (2012a) Fluorescence parameters as early indicators of light stress in barley. J Photochem Photobiol B 112:1-6

Kalaji MH, Govindjee, Goltsev V, Bosa K, Allakhverdiev SI, Strasser $\mathrm{R}$ (2012b) Experimental in vivo measurements of light emission in plants: a perspective dedicated to David Walker. Photosynth Res 114:69-96

Kalaji MH, Oukarroum A, Alexandrov V, Kouzmanova M, Brestič M, Živčák M, Samborska IA, Cetner MD, Allakhverdiev SI, Vasilij Goltsev V (2014a) Identification of nutrient deficiency in maize and tomato plants by in vivo chlorophyll $a$ fluorescence measurements. Plant Physiol Biochem 81:16-25

Kalaji HM, Schansker G, Ladle RJ, Goltsev V, Bosa K, Allakhverdiev SI, Brestič M, Bussotti F, Calatayud A, Dąbrowski Elsheery NI, Ferroni L, Guidi L, Hogewoning SW, Jajoo A, Misra AN, Nebauer SG, Pancaldi S, Penella C, Poli DB, Pollastrini M, Romanowska-Duda ZB, Rutkowska B, Serôdio J, Suresh K, Szulc W, Tambussi E, Yanniccari M, Živčák M (2014b) Frequently Asked Questions about chlorophyll fluorescence: practical issues. Photosynth Res 122:121-158

Kalaji HM, Goltsev VN, Żuk-Golaszewska K, Živčák M, Brestič M (2016) Application of Chlorophyll Fluorescence in Understanding Plant Performance. CRC Press, Florida

Kaňa R, Spundová P, Ilík P, Lazár D, Klem K, Tomek P, Naus J, Prásil O (2004) Effect of herbicide clomazone on photosynthetic processes in primary barley (Hordeum vulgare L.) leaves. Pestic Biochem Physiol 78:161-170

Karapetyan NV (2004) Interaction of pigment-protein complexes within aggregates stimulates dissipation of excess energy. Biochem (Moscow) 69:1299-1304

Karapetyan NV, Bolychevtseva YV, Yurina NP, Terekhova IV, Shubin VV, Brecht M (2014) Long-wavelength chlorophylls in photosystem I of cyanobacteria: origin, localization, and functions. Biochem (Moscow) 79:213-220

Kasajima I, Takahara K, Kawai-Yamada M, Uchimiya H (2009) Estimation of the relative sizes of rate constants for chlorophyll de-excitation processes through comparison of inverse fluorescence intensities. Plant Cell Physiol 50:1600-1616

Katsumata M, Takeuchi A, Kazumura K, Koike T (2008) New feature of delayed luminescence: preillumination-induced concavity and convexity in delayed luminescence decay curve in the green alga Pseudokirchneriella subcapitata. J Photochem Photobiol B 90:152-162

Kautsky H, Hirsch A (1931) Neue Versuche zur Kohlensäureassimilation. Naturwissenschaften 19:964 [in German]

Kautsky H, Appel W, Amann H (1960) Chlorophyllfluoreszenz und Kohlensäure-assimilation: XIII. Die Fluoreszenzkurve und die Photochemie der Pflanze. Biochem Z 322:277-292 [in German]

Keränen M, Aro E-M, Tyystjärvi E (2003) Automatic plant identification with chlorophyll fluorescence fingerprinting. Precis Agric 4:53-67

Kiani SP, Maury P, Sarrafi A, Grieu P (2008) QTL analysis of chlorophyll fluorescence parameters in sunflower (Helianthus annuи L.) under well-watered and water-stressed conditions. Plant Sci 175:565-573

Kirchhoff H, Horstmann S, Weis E (2000) Control of the photosynthetic electron transport by $\mathrm{PQ}$ diffusion microdomains in thylakoids of higher plants. Biochim Biophys Acta 1459:148-168

Kirchhoff H, Hinz HJ, Rösgen J (2003) Aggregation and chlorophyll $a$ fluorescence quenching of the light harvesting complex II from spinach in vitro. Biochim Biophys Acta 1606:105-116

Kirova M, Ceppi G, Chernev P, Goltsev V, Strasser R (2009) Using artificial neural networks for plant taxonomic determination based on chlorophyll fluorescence induction curves. Biotechnol Biotechnol Equip 23:941-945 
Klughammer C, Schreiber U (1994) An improved method, using saturating light pulses, for the determination of photosystem I quantum yield via $\mathrm{P} 700^{+}$-absorbance changes at $830 \mathrm{~nm}$. Planta 192:261-268

Knoetzel J, Bossmann B, Grimme H (1998) Chlorina and viridis mutants of barley (Hordeum vulgare L.) allow assignment of long-wavelength chlorophyll forms to individual Lhca proteins of Photosystem I in vivo. FEBS Lett 436:339-342

Kok B, Forbush B, McGloin M (1970) Cooperation of charges in photosynthetic $\mathrm{O}_{2}$ evolution; I. A linear four-step mechanism. Photochem Photobiol 11:467-475

Komayama K, Khatoon M, Takenaka D, Horie J, Yamashita A, Yoshioka M, Nakayama Y, Yoshida M, Ohira S, Morita N, Velitchkova M, Enami I, Yamamoto Y (2007) Quality control of photosystem II: cleavage and aggregation of heat-damaged D1 protein in spinach thylakoids. Biochim Biophys Acta 1767:838-846

Kong S-G, Wada M (2014) Recent advances in understanding the molecular mechanism of chloroplast photorelocation movement. Biochim Biophys Acta 1837:522-530

Konishi A, Eguchi A, Hosoi F, Omasa K (2009) 3D monitoring spatio-temporal effects of herbicide on a whole plant using combined range and chlorophyll $a$ fluorescence imaging. Funct Plant Biol 36:874-879

Konstantinova P, Van der Schoor R, van den Bulk RW, Jalink H (2002) Chlorophyll fluorescence sorting as a method for improvement of barley (Hordeum vulgare L.) seed health and germination. Seed Sci Technol 30:411-421

Kopriva S, Rennenberg H (2004) Control of sulphate assimilation and glutathione synthesis: interaction with $\mathrm{N}$ and $\mathrm{C}$ metabolism. J Exp Bot 55:1831-1842

Kornyeyev D, Hendrickson L (2007) Energy partitioning in photosystem II complexes subjected to photoinhibitory treatment. Funct Plant Biol 34:214-220

Kornyeyev D, Guadagno CR, D'Ambrosio N (2013) Assessment of energy partitioning in PSII complexes using chlorophyll fluorescence: reviewing the different approaches toward the definition of a unified method. Photosynthetica 51:477-480

Kotakis C, Kyeridou A, Manetas Y (2014) Photosynthetic electron flow during leaf senescence: evidence for a preferential maintenance of photosystem I activity and increased cyclic electron flow. Photosynthetica 52:413-420

Krall JP, Edwards GE (1990) Quantum yields of photosystem II electron transport and carbon dioxide fixation in $\mathrm{C} 4$ plants. Aust J Plant Physiol 17:579-588

Krall JP, Edwards GE (1992) Relationship between photosystem II activity and $\mathrm{CO}_{2}$ fixation in leaves. Physiol Plant 86:180-187

Kramer DM, Robinson HR, Crofts AR (1990) A portable multi-flash kinetic fluorimeter for measurement of donor and acceptor reactions of photosystems 2 in leaves of intact plants under field conditions. Photosynth Res 26:181-193

Kramer DM, Sacksteder CA, Cruz JA (1999) How acidic is the lumen? Photosynth Res 60:151-163

Kramer DM, Avenson TJ, Edwards GE (2004a) Dynamic flexibility in the light reactions of photosynthesis governed by both electron and proton transfer reactions. Trends Plant Sci 9:349-357

Kramer DM, Johnson G, Kiirats O, Edwards GE (2004b) New fluorescence parameters for the determination of $\mathrm{Q}_{\mathrm{A}}$ redox state and excitation energy fluxes. Photosynth Res 79:209-218

Krause G, Weis E (1991) Chlorophyll fluorescence and photosynthesis: the basics. Annu Rev Plant Physiol Plant Mol Biol 42:313-349

Krause GH, Vernotte C, Briantais J-M (1982) Photoinduced quenching of chlorophyll fluorescence in intact chloroplasts and algae; resolution into two components. Biochim Biophys Acta 679:116-124

Krausz E, Hughes JL, Smith PJ, Pace RJ, Peterson Årsköld S (2005) Assignment of the low-temperature fluorescence in oxygenevolving photosystem II. Photosynth Res 84:193-199

Krieger A, Weis E, Demeter S (1993) Low-pH-induced $\mathrm{Ca}^{2+}$ ion release in the water-splitting system is accompanied by a shift in the midpoint redox potential of the primary quinone acceptor $\mathrm{Q}_{\mathrm{A}}$. Biochim Biophys Acta 1144:411-418

Krieger-Liszkay A (2004) Singlet oxygen production in photosynthesis. J Exp Bot 56:337-346

Krieger-Liszkay A, Rutherford AW (1998) Influence of herbicide binding on the redox potential of the quinone acceptor in photosystem II: relevance to photodamage and phytotoxicity. Biochemistry 37:17339-17344

Krieger-Liszkay A, Fufezan C, Trebst A (2008) Singlet oxygen production in photosystem II and related protetction mechanism. Photosynth Res 98:551-564

Kromer S (1995) Respiration during photosynthesis. Annu Rev Plant Physiol Plant Mol Biol 46:45-70

Ku MSB, Wu J, Dai Z, Scott RA, Chu C, Edwards GE (1991) Photosynthetic and photorespiratory characteristics of Flaveria species. Plant Physiol 96:518-528

Kuang TY, Argyroudi-Akoyunoglou JH, Nakatani HY, Watson J, Arntzen CJ (1984) The origin of the long-wavelength fluorescence emission band (77 K) from photosystem I. Arch Biochem Biophys 235:618-627

Kumar K, Dahms H, Lee J, Kim H, Lee W, Shin K (2014) Algal photosynthetic responses to toxic metals and herbicides assessed by chlorophyll a fluorescence. Ecotoxicol Environ Safety 104:51-71

Kvíderová J (2010) Rapid algal toxicity assay using variable chlorophyll fluorescence for Chlorella kessleri (chlorophyta). Environ Toxicol 25:554-563

Kyle DJ, Baker NR, Arntzen CJ (1983) Spectral characterization of photosystem I fluorescence at room temperature using thylakoid protein phosphorylation of Chlamydomonas reinhardtii. Photobiochem Photobiophys 5:71-78

Laisk A, Edwards GE (1998) Oxygen and electron flow in $\mathrm{C}_{4}$ photosynthesis: Mehler reaction, photorespiration and $\mathrm{CO}_{2}$ concentration in the bundle sheath. Planta 205:632-645

Laisk A, Oja V (1998) Dynamics of leaf photosynthesis. CSIRO Publishing, Collingwood

Laisk A, Nedbal L, Govindjee (2009) Photosynthesis in Silico; understanding complexity from molecules to ecosystems. Advances in Photosynthesis and Respiration, vol 25. Springer, Dordrecht

Lange OL, Lösch R, Schulze E-D, Kappen L (1971) Responses of stomata to changes in humidity. Planta 100:76-86

Larcher W (2000) Temperature stress and survival ability of Mediterranean sclerophyllous plants. Plant Biosyst 134:279-295

Latimer P, Bannister TT, Rabinowitch E (1956) Quantum yields of fluorescence of plant pigments. Science 124:585-586

Lavergne J, Trissl H-W (1995) Theory of fluorescence induction in photosystem II: derivation of analytical expression in a model including exciton-radical-pair equilibrium and restricted energy transfer between photosynthetic units. Biophys J 68:2474-2492

Lavergne J, Bouchaud J-P, Joliot P (1992) Plastoquinone compartmentation in chloroplasts. II. Theoretical aspects. Biochim Biophys Acta 1101:13-22

Lavorel J (1975) Luminescence. In: Govindjee (ed) Bioenergetics of photosynthesis. Academic Press, New York, pp 223-317

Lavorel J, Lavergne J, Etienne AL (1982) A reflection of several problems of luminescence in photosynthetic systems. Photobiochem Photobiophys 3:287-314 
Law RD, Crafts-Brandner SJ (1999) Inhibition and acclimation of photosynthesis to heat stress is closely correlated with activation of ribulose-1,5-bisphosphate carboxylase/oxygenase. Plant Physiol 120:173-181

Lazár D (1999) Chlorophyll $a$ fluorescence induction. Biochim Biophys Acta 1412:1-28

Lazár D (2003) Chlorophyll $a$ fluorescence rise induced by high light illumination of dark-adapted plant tissue studied by means of a model of photosystem II and considering photosystem II heterogeneity. J Theor Biol 220:469-503

Lazár D (2006) The polyphasic chlorophyll $a$ fluorescence rise measured under high intensity of exciting light. Funct Plant Biol 33:9-30

Lazár D (2013) Simulations show that a small part of variable chlorophyll $a$ fluorescence originates in photosystem $\mathrm{I}$ and contributes to overall fluorescence rise. $J$ Theor Biol 335:249-264

Lazár D (2015) Parameters of photosynthetic energy partitioning. J Plant Physiol 175:131-147

Lazár D (2016) A word or two about chlorophyll fluorescence and its relation to photosynthesis research; a text for Ph.D. students. doi:10.13140/RG.2.1.4243.0329

Lazár D, Ilík P (1997) High-temperature induced chlorophyll fluorescence changes in barley leaves; comparison of the critical temperatures determined from fluorescence induction and from fluorescence temperature curve. Plant Sci 124:159-164

Lazár D, Nauš J (1998) Statistical properties of chlorophyll fluorescence induction parameters. Photosynthetica 35:121-127

Lazár D, Pospíšil P (1999) Mathematical simulation of chlorophyll $a$ fluorescence rise measured with 3-(3',4'-dichlorophenyl)-1,1dimethylurea-treated barley leaves at room and high temperatures. Eur Biophys J 28:468-477

Lazár D, Schansker G (2009) Models of chlorophyll $a$ fluorescence transients. In: Laisk A, Nedbal L, Govindjee (eds) Photosynthesis in silico; understanding complexity from molecules to ecosystems. Advances in photosynthesis and respiration, vol 25. Springer, Dordrecht, pp 85-123

Lazár D, Ilík P, Nauš J (1997a) An appearance of K-peak in fluorescence induction depends on the acclimation of barley leaves to higher temperatures. J Luminesc 72-74:595-596

Lazár D, Nauš J, Matoušková M, Flašarová M (1997b) Mathematical modeling of changes in chlorophyll fluorescence induction caused by herbicides. Pest Biochem Physiol 57:200-210

Lazár D, Brokeš M, Nauš J, Dvořák L (1998) Mathematical modelling of 3-(3',4'-dichlorophenyl)-1,1-dimethylurea action in plant leaves. J Theor Biol 191:79-86

Lazár D, Tomek P, Ilík P, Nauš J (2001) Determination of the antenna heterogeneity of Photosystem II by direct simultaneous fitting of several fluorescence rise curves measured with DCMU at different light intensities. Photosynth Res 68:247-257

Lazár D, Sušila P, Nauš J (2005) Statistical properties of parameters evaluated from the O-J-I-P chlorophyll $a$ fluorescence transients and their change upon high temperature stress. In: van Est A, Bruce D (eds) Photosynthesis: fundamental aspects to global perspectives. Allen Press, Lawrence, pp 563-566

Legendre P, Legendre L (2012) Numerical ecology, vol 3. Elsevier, Amsterdam

Leipner J, Oxborough K, Baker NR (2001) Primary sites of ozoneinduced perturbations of photosynthesis in leaves: identification and characterization in Phaseolus vulgaris using high resolution chlorophyll fluorescence imaging. J Exp Bot 52:1689-1696

Leustek T, Martin MN, Bick J-A, Davies JP (2000) Pathways and regulation of sulfur metabolism revealed through molecular and genetic studies. Annu Rev Plant Physiol Plant Mol Biol 51:141-165
Lewis S, Donkin ME, Depledge MH (2001) Hsp70 expression in Enteromorpha intestinalis (Chlorophyta) exposed to environmental stress. Aquat Bot 51:277-291

Lichtenthaler HK (1988) Applications of chlorophyll fluorescence. Kluwer Academic, Dordrecht

Lichtenthaler HK (2013) Chlorophyll fluorescence kinetics, photosynthetic activity, and pigment composition of blue-shade and half-shade leaves as compared to sun and shade leaves of different trees. Photosynth Res 117:355-366

Lichtenthaler HK, Buschmann C, Döll M, Fietz HJ, Bach T, Kozel U, Meier D, Rahmsdorf U (1981) Photosynthetic activity, chloroplast ultrastructure, and leaf characteristics of high-light and low-light plants and of sun and shade leaves. Photosynth Res 2:115-141

Lichtenthaler HK, Buschmann C, Rinderle U, Schmuck G (1986) Application of chlorophyll fluorescence in ecophysiology. Radiat Environmental Biophys 25:297-308

Lichtenthaler HK, Buschmann C, Knapp M (2005a) How to correctly determine the different chlorophyll fluorescence parameters and the chlorophyll fluorescence decrease ratio $\mathrm{R}_{\mathrm{Fd}}$ of leaves with the PAM fluorometer. Photosynthetica 43:379-393

Lichtenthaler HK, Langsdorf G, Lenk S, Buschmann C (2005b) Chlorophyll fluorescence imaging of photosynthetic activity with the flash-lamp fluorescence imaging system. Photosynthetica 43:355-369

Lichtenthaler HK, Ač A, Marek MV, Kalina J, Urban O (2007) Differences in pigment composition, photosynthetic rates and chlorophyll fluorescence images of sun and shade leaves of four tree species. Plant Physiol Biochem 45:577-588

Logan BA, Adams WW, Demmig-Adams B (2007) Viewpoint: avoiding common pitfalls of chlorophyll fluorescence analysis under field conditions. Funct Plant Biol 34:853-859

Long SP, Humphries S (1994) Photoinhibition of photosynthesis in nature. Annu Rev Plant Physiol Plant Mol Biol 45:633-662

Long SP, Ainsworth EA, Leakey ADB, Nösberger J, Ort DR (2006) Food for thought: lower-than-expected crop yield stimulation with rising $\mathrm{CO}_{2}$ concentrations. Science 312:1918-1921

Losciale P, Oguchi R, Hendrickson L, Hope AB, Corelli Grappadelli L, Chow WS (2008) A rapid, whole-tissue determination of the functional fraction of photosystem II after photoinhibition of leaves based on flash-induced P700 redox kinetics. Physiol Plant 132:23-32

Losciale P, Chow WS, Corelli Grappadelli L (2010) Modulating the light environment with the peach 'asymmetric orchard': effects on gas exchange performances, photoprotection, and photoinhibition. J Exp Bot 61:1177-1192

Losciale P, Manfrini L, Morandi B, Pierpaoli E, Zibordi M, Stellaci AM, Salvati L, Grappadelli LC (2015) A multivariate approach for assessing leaf photo-assimilation performance using the $\mathrm{I}_{\mathrm{PL}}$ index. Physiol Plant 154:609-620

Lu C, Zhang J (1998) Effects of water stress on photosynthesis, chlorophyll fluorescence and photoinhibition in wheat plants. Aust J Plant Physiol 25:883-892

Lu C, Zhang J (1999) Effects of water stress on photosystem II photochemistry and its thermostability in wheat plants. J Exp Bot 50:1199-1206

Lu Q, Lu C, Zhang J, Kuang T (2002) Photosynthesis and chlorophyll $a$ fluorescence during flag leaf senescence of field-grown wheat plants. J Plant Physiol 159:1173-1178

Lunde C, Jensen PE, Haldrup A, Knoetzel J, Scheller HV (2000) The PSI-H subunit of photosystem I is essential for state transitions in plant photosynthesis. 408:613-615

Macinnis-Ng CMO, Ralph PJ (2003) Short-term response and recovery of Zostera capricorni photosynthesis after herbicide exposure. Aqua Bot 76:1-15 
Maldonado-Rodriguez R, Pavlov S, Gonzalez A, Oukarroum A, Strasser RJ (2003) Can machines recognise stress in plants? Environ Chem Lett 1:201-205

Malenovsky Z, Mishra KB, Zemek F, Rascher U, Nedbal L (2009) Scientific and technical challenges in remote sensing of plant canopy reflectance of fluorescence. J Exp Bot 60:2987-3004

Malkin S (1966) Fluorescence induction studies in isolated chloroplasts. I. Kinetic analysis of the fluorescence intensity dependence on time. Biochim Biophys Acta 126:433-442

Malkin S (1979) Delayed luminescence. In: Trebst A, Avron M (eds) Photosynthesis I. Photosynthetic Electron Transport and Photophosphorylation. Academic Press, New York, pp 473-491

Malkin S, Barber J (1978) Induction patterns of delayed luminescence from isolated chloroplasts. I. Response of delayed luminescence to changes in the prompt fluorescence yield. Biochim Biophys Acta 502:524-541

Malkin S, Bilger W, Schreiber U (1994) The relationship between millisecond luminescence and fluorescence in tobacco leaves during the induction period. Photosynth Res 39:57-66

Mar T, Brebner J, Roy G (1975) Induction kinetics of delayed light emission in spinach chloroplasts. Biochim Biophys Acta 376:345-353

Martínez-Ferri E, Manrique E, Valladares F, Balaguer L (2004) Winter photoinhibition in the field involves different processes in four co-occurring Mediterranean tree species. Tree Physiol 24:981-990

Marwood CA, Solomon KR, Greenberg BM (2001) Chlorophyll fluorescence as a bioindicator of effects on growth in aquatic macrophytes from mixtures of polycyclic aromatic hydrocarbons. Environ Toxicol Chem 20:890-898

Matsubara S, Chow WS (2004) Population of photoinactivated Photosystem II reaction centers characterized by chlorophyll a fluorescence lifetime in vivo. Proc Natl Acad Sci USA 101:18234-18239

Matsubara S, Chen Y-C, Caliandro R, Govindjee, Clegg RM (2011) Photosystem II fluorescence lifetime imaging in avocado leaves: contributions of the lutein-epoxide and violaxanthin cycles to fluorescence quenching. J Photochem Photobiol B 104:271-284

Maxwell K, Johnson GN (2000) Chlorophyll fluorescence-a practical guide. J Exp Bot 51:659-668

McCormac DJ, Bruce D, Greenberg BM (1994) State transitions, light-harvesting antenna phosphorylation and light-harvesting antenna migration in vivo in the higher plant Spirodela oligorrhiza. Biochim Biophys Acta 1187:301-312

McCree KJ (1972) The action spectrum, absorptance and quantum yield of photosynthesis in crop plants. Agric Meteorol 9:191-216

Medrano H, Escalona JM, Bota J, Gulías J, Flexas J (2002) Regulation of photosynthesis of $\mathrm{C} 3$ plants in response to progressive drought: stomatal conductance as a reference parameter. Ann Bot 89:895-905

Melgar JC, Guidi L, Remorini Agati G, Degl'Innocenti E, Castelli S, Baratto MC, Faraloni C, Tattini M (2009) Antioxidant defences and oxidative damage in salt-treated olive plants under contrasting sunlight irradiance. Tree Physiol 29:1187-1198

Melis A, Homann PH (1975) Kinetic analysis of the fluorescence induction in 3-(3,4- dichlorophenyl)-1,1-dimethylurea poisoned chloroplasts. Photochem Photobiol 21:431-437

Melis A, Homann PH (1976) Heterogeneity of the photochemical centers in system II of chloroplasts. Photochem Photobiol 23:343-350

Merkell U, Rathke GW, Schuster C, Warnstorff K, Diepenbrock W (2004) Use of glufosinate-ammonium to control cruciferous weed species in glufosinate-resistant winter oilseed rape. Field Crop Res 85:237-249
Merz D, Geyer M, Moss DA, Ache HJ (1996) Chlorophyll fluorescence biosensor for the detection of herbicides. Fresenius J Anal Chem 354:299-305

Messinger J, Renger G (1990) The reactivity of hydrazine with photosystem II strongly depends on the redox state of the water oxidizing system. FEBS Lett 277:141-146

Messinger J, Renger G (1993) Generation, oxidation by the oxidized form of the tyrosine of polypeptide D2, and possible electronic configuration of the redox states S0, S-1, and S-2 of the water oxidase in isolated spinach thylakoids. Biochemistry 32:9379-9386

Meyer S, Genty B (1999) Heterogeneous inhibition of photosynthesis over the leaf surface of Rosa rubiginosa $\mathrm{L}$. during water stress and abscisic acid treatment: induction of a metabolic component by limitation of $\mathrm{CO}_{2}$ diffusion. Planta 210:126-131

Misra AN, Biswal AK (2000) Thylakoid membrane protein kinase activity as a signal transduction pathway in chloroplasts. Photosynthetica 38:323-332

Misra AN, Srivastava A, Strasser RJ (2001a) Fast chlorophyll $a$ fluorescence kinetic analysis for the assessment of temperature and light effects: a dynamic model for stress recovery phenomena. Photosynthesis: PS2001. CSIRO Publ., Melbourne, Australia $\mathrm{S} 3-007$

Misra AN, Srivastava A, Strasser RJ (2001b) Utilisation of fast chlorophyll $a$ fluorescence technique in assessing the salt/ion sensitivity of mung bean and brassica seedlings. J Plant Physiol 158:1173-1181

Misra AN, Latowski D, Strzalka K (2006) The xanthophyll cycle activity in kidney bean and cabbage leaves under salinity stress. Russ J Plant Physiol 53:102-109

Misra AN, Misra M, Singh R (2012) Chlorophyll fluorescence in plant biology. In: Misra AN (ed) Biophysics. Intech Europe, Croatia, pp 171-192

Mittler R (2002) Oxidative stress, antioxidants and stress tolerance. Trends Plant Sci 7:405-410

Mohanty N, Vass I, Demeter S (1989) Copper toxicity affects photosystem II electron transport at the secondary quinone acceptor, Qв. Plant Physiol 90:175-179

Mukerji I, Sauer K (1990) Optical Spectroscopy on a Photosystem I Antenna Complex. In: Baltscheffsky M (ed) Current research in photosynthesis, vol II. Kluwer Academic Publishers, Dordrecht, pp 321-324

Mulkidjanian AY (2010) Activated Q cycle as a common mechanism for cytochrome bc1 and cytochrome b6f complexes. Biochim Biophys Acta 1797:1858-1868

Muller R, Schreiber U, Escher BI, Quayle P, Bengtson Nash SM, Mueller JF (2008) Rapid exposure assessment of PSII herbicides in surface water using a novel chlorophyll $a$ fluorescence imaging assay. Sci Total Environ 401:51-59

Müller P, Li X-P, Niyogi KK (2001) Non-photochemical quenching; a response to excess light energy. Plant Physiol 125:1558-1566

Mullet JE, Burke JJ, Arntzen CJ (1980) Chlorophyll proteins of photosystem I. Plant Physiol 65:814-822

Munday JC, Govindjee (1969) Light-induced changes in the fluorescence yield of chlorophyll $a$ in vivo; III. The dip and the peak in the fluorescence transient of Chlorella pyrenoidosa. Biophys $\mathrm{J}$ 9:1-21

Munekage YN, Eymery F, Rumeau D, Cuiné S, Oguri M, Nakamura N, Yakota A, Genty B, Peltier G (2010) Elevated expression of PGR5 and NDH-H in bundle sheath chloroplasts in $\mathrm{C}_{4}$ Flaveria species. Plant Cell Physiol 51:664-668

Murakami Y, Tsuyama M, Kobayashi Y, Kodama H, Iba K (2000) Trienoic fatty acids and plant tolerance of high temperature. Science 287:476-479

Murata N, Nishimura M, Takamiya A (1966a) Fluorescence of chlorophyll in photosynthetic systems; II. Induction of 
fluorescence in isolated spinach chloroplasts. Biochim Biophys Acta 120:23-33

Murata N, Nishimura M, Takamiya A (1966b) Fluorescence of chlorophyll in photosynthetic systems. III. Emission and action spectra of fluorescence-three emission bands of chlorophyll $a$ and the energy transfer between two pigment systems. Biochim Biophys Acta 126:234-243

Murchie EH, Lawson T (2013) Chlorophyll fluorescence analysis: a guide to good practice and understanding some new applications. J Exp Bot 64:3983-3998

Nagy V, Tengölics R, Schansker G, Rákhely G, Kovács KL, Garab G, Tóth SZ (2012) Stimulatory effect of ascorbate, the alternative electron donor of photosystem II, on the hydrogen production of Chlamydomonas reinhardtii. Int J Hydrog Energ 37:8864-8871

Nakatani HY, Ke B, Dolan E, Arntzen CJ (1984) Identity of the photosystem II reaction center polypeptide. Biochim Biophys Acta 765:347-352

Nauš J, Šmecko S, Špundová M (2016) Chloroplast avoidance movement as a sensitive indicator of relative water content during leaf dessication in the dark. Photosynth Res 129:217-225

Nedbal L, Whitmarsh J (2004) Chlorophyll fluorescence imaging of leaves and fruits. In: Papageorgiou GC, Govindjee (eds) Advances in photosynthesis and respiration series chlorophyll fluorescence: a signature of photosynthesis, vol 19. Springer, Dordrecht, pp 389-407

Nedbal L, Soukupová J, Whitmarsh J, Trtílek M (2000) Postharvest imaging of chlorophyll fluorescence from lemons can be used to predict fruit quality. Photosynthetica 38:571-579

Nejad AR, Harbinson J, van Meeteren U (2006) Dynamics of spatial heterogeneity of stomatal closure in Tradescantia virginiana altered by growth at high relative humidity. J Exp Bot 57:3669-3678

Neubauer C, Schreiber U (1987) The polyphasic rise of chlorophyll fluorescence upon onset of the strong continuous illumination: I. Saturation characteristics and partial control by the photosystem II acceptor side. Z Naturforsch 42c:1246-1254

Neyra CH, Hageman RH (1974) Dependence of nitrite reduction on electron transport in chloroplasts. Plant Physiol 54:480-484

Nilkens M, Kress E, Lambrev P, Miloslavina Y, Müller M, Holzwarth AR, Jahns P (2010) Identification of a slowly inducible zeaxanthin-dependent component of non-photochemical quenching of chlorophyll fluorescence generated under steady-state conditions in Arabidopsis. Biochim Biophys Acta 1797:466-475

Noctor G, Foyer CH (1998) Ascorbate and glutathione: keeping active oxygen under control. Annu Rev Plant Physiol Plant Mol Biol 49:249-279

Oberhuber W, Dai Z-Y, Edwards GE (1993) Light dependence of quantum yields photosystem II and $\mathrm{CO}_{2}$ fixation in $\mathrm{C}_{3}$ and $\mathrm{C}_{4}$ plants. Photosynth Res 35:265-274

Ögren E, Rosenqvist E (1992) On the significance of photoinhibition of photosynthesis in the field and its generality among species. Photosynth Res 33:63-71

Oh M-H, Moon Y-H, Lee C-H (2003) Increased stability of LHCII by aggregate formation during dark-induced leaf senescence in the Arabidopsis mutant ore10. Plant Cell Physiol 44:1368-1377

Oja V, Laisk A (2012) Photosystem II antennae are not energetically connected: evidence based on flash-induced $\mathrm{O}_{2}$ evolution and chlorophyll fluorescence in sunflower leaves. Photosynth Res 114:15-28

Olesen CF, Cedergreen N (2010) Glyphosate uncouples gas exchange and chlorophyll fluorescence. Pest Manag Sci 66:536-542

Oukarroum A, El Madidi S, Schansker G, Strasser RJ (2007) Probing the responses of barley cultivars (Hordeum vulgare L.) by chlorophyll $a$ fluorescence OLKJIP under drought stress and rewatering. Environ Exp Bot 60:438-446

Oukarroum A, Schansker G, Strasser RJ (2009) Drought stress effects on photosystem I content and photosystem II thermotolerance analyzed using Chl $a$ fluorescence kinetics barley varieties differing in their drought tolerance. Physiol Plant 137:188-199

Oukarroum A, Strasser RJ, Schansker G (2012) Heat stress response of the lichen Parmelia tiliacea in the dry and the wet state: differences and similarities with the heat stress response of higher plants. Photosynth Res 111:303-314

Oxborough K (2004) Using chlorophyll a fluorescence imaging to monitor photosynthetic performance. In: Papageorgiou GC, Govindjee (eds) Chl $a$ fluorescence: a signature of photosynthesis, advances in photosynthesis and respiration, vol 19. Springer, Dordrecht, pp 409-428

Oxborough K, Baker NR (1997) Resolving chlorophyll $a$ fluorescence images of photosynthetic efficiency into photochemical and nonphotochemical components: calculation of $\mathrm{qP}$ and $\mathrm{Fv}^{\prime} / \mathrm{Fm}$ ' without measuring Fo'. Photosynth Res 54:135-142

Pålsson LO, Tjus SE, Andersson B, Gillbro T (1995) Energy-transfer in photosystem-I: time-resolved fluorescence of the native photosystem-I complex and its core complex. Chem Phys 194:291-302

Pantaleoni L, Ferroni L, Baldisserotto C, Aro EM, Pancaldi S (2009) Photosystem II organisation in chloroplasts of Arum italicum leaf depends on tissue location. Planta 230:1019-1031

Pantazi XE, Moshou D, Mouazen AM, Kuang B, Alexandridis T (2014) Application of supervised self organising models for wheat yield prediction. In: Iliadis L, Maglogiannis I, Papadopoulos $\mathrm{H}$ (eds) Artificial intelligence applications and innovations. Springer, Berlin, pp 556-565

Papageorgiou GC, Govindjee (eds) (2004) Chl $a$ fluorescence: a signature of photosynthesis, advances in photosynthesis and respiration, vol 19. Springer, Dordrecht

Pastenes C, Horton P (1996) Effect of high temperature on photosynthesis in beans. 1. Oxygen evolution and chlorophyll fluorescence. Plant Physiol 112:1245-1251

Paul M, Foyer CH (2001) Sink regulation of photosynthesis. J Exp Bot 52:1383-1400

Penella C, Landi M, Guidi L, Nebauer SG, Pellegrini E, San Bautista A, Remorini D, Nali C, Lopez-Galarza S, Calatayud A (2016) Salt-tolerant rootstock increases yield of pepper under salinity through maintenance of photosynthetic performance and sink strength. J Plant Physiol 193:1-11

Perreault F, Oukarroum A, Pirastru L, Sirois L, Matias GW, Popovic R (2010) Evaluation of copper oxide nanoparticles toxicity using chlorophyll a fluorescence imaging in Lemna gibba. J Bot. doi: $10.1155 / 2010 / 763142$

Pesaresi P, Hertle A, Pribil M, Kleine T, Wagner R, Strissel H, Ihnatowicz A, Bonardi V, Scharfenberg M, Schneider A, Pfannschmidt T, Leister D (2009) Arabidopsis STN7 kinase provides a link between short- and long-term photosynthetic acclimation. Plant Cell 21:2402-2423

Peterson RB, Havir EA (2003) Contrasting modes of regulation of PS II light utilization with changing irradiance in normal and psbS mutant leaves of Arabidopsis thaliana. Photosynth Res 75:57-70

Peterson RB, Havir EA (2004) The multiphasic nature of nonphotochemical quenching: implications for assessment of photosynthetic electron transport based on chlorophyll fluorescence. Photosynth Res 82:95-107

Peterson RB, Oja V, Laisk A (2001) Chlorophyll fluorescence at 680 and $730 \mathrm{~nm}$ and leaf photosynthesis. Photosynth Res 70:185-196

Peterson RB, Oja V, Eichelmann H, Bichele I, Dall'Osto L, Laisk A (2014) Fluorescence F0 of photosystems II and I in developing $\mathrm{C} 3$ and $\mathrm{C} 4$ leaves, and implications on regulation of excitation balance. Photosynth Res 122:41-56

Petrouleas V, Crofts AR (2005) The iron-quinone acceptor complex. In: Wydrzynski TJ, Satoh K (eds) Photosystem II: the lightdriven water:plastoquinone oxidoreductase, advances in photosynthesis and respiration, vol 22. Springer, Dordrecht, pp 177-206 
Pfündel E (1998) Estimating the contribution of photosystem I to total leaf chlorophyll fluorescence. Photosynth Res 56:185-195

Pfündel E, Pfeffer M (1997) Modification of photosystem I light harvesting of bundle-sheath chloroplasts occurred during the evolution of NADP-malic enzyme $\mathrm{C} 4$ photosynthesis. Plant Physiol 114:145-152

Pfündel EE, Klughammer C, Meister A, Cerovic ZG (2013) Deriving fluorometer-specific values of relative PSI fluorescence intensity from quenching of F0 fluorescence in leaves of Arabidopsis thaliana and Zea mays. Photosynth Res 114:189-206

Pogson BJ, Niyogio KK, Björkman O, DellaPenna D (1998) Altered xanthophyll compositions adversely affect chlorophyll accumulation and nonphotochemical quenching in Arabidopsis mutants. Proc Natl Acad Sci USA 95:13324-13329

Pollastrini M, Di Stefano V, Ferretti M, Agati G, Grifoni D, Zipoli G, Orlandini S, Bussotti F (2011) Influence of different light intensity regimes on leaf features of Vitis vinifera L. in ultraviolet radiation filtered condition. Environ Exp Bot 73:108-115

Pollastrini M, Desotgiu R, Camin F, Ziller L, Marzuoli R, Gerosa G, Bussotti F (2013) Intra-annual pattern of photosynthesis, growth and stable isotope partitioning in a poplar clone subjected to ozone and water stress. Water Air Soil Pollut 224:1761-1772

Pollastrini M, Holland V, Brüggemann W, Koricheva J, Jussila I, Scherer-Lorenzen M, Berger S, Bussotti F (2014) Interactions and competition processes among tree species in young experimental mixed forests, assessed with chlorophyll fluorescence and leaf morphology. Plant Biol 16:323-331

Pollet B, Steppe K, van Labeke M-C, Lemeur R (2009) Diurnal cycle of chlorophyll fluorescence in Phalaenopsis. Photoynthetica 47:309-312

Popovic R, Dewez D, Juneau P (2003) Applications of chlorophyll fluorescence in ecotoxicology: heavy metals, herbicides, and air pollutants. In: DeEll JR, Toivonen PMA (eds) Practical applications of chlorophyll fluorescence in plant biology. Kluwer Academic Publishers, Dordrecht, pp 151-184

Porcar-Castell A, Tyystjärvi E, Atherton J, van der Tol C, Flexas J, Pfündel EE, Moreno J, Frankenberg C, Berry JA (2014) J Exp Bot 65:4065-4095

Pospíčil P, Haumann M, Dittmer J, Solé VA, Dau H (2003) Stepwise transition of the tetra-manganese complex of photosystem II to a binuclear $\mathrm{Mn}_{2}(\mu-\mathrm{O})_{2}$ complex in response to a temperature jump: a time-resolved structural investigation employing X-ray absorption spectroscopy. Biophys J 84:1370-1386

Quick WP, Horton P (1984) Studies on the induction of chlorophyll fluorescence in barley protoplasts; II. Resolution of fluorescence quenching by redox state and the transthylakoid $\mathrm{pH}$ gradient. Proc R Soc Lond B 220:371-382

Quick WP, Stitt M (1989) An examination of factors contributing to non-photochemical quenching of chlorophyll fluorescence in barley leaves. Biochim Biophys Acta 977:287-296

Quilliam RS, Swarbrick PJ, Scholes JD, Rolfe SA (2006) Imaging photosynthesis in wounded leaves of Arabidopsis thaliana. J Exp Bot 57:55-69

Radenovic C, Markovic D, Jeremic M (1994) Delayed chlorophyll fluorescence in plant models. Photosynthetica 30:1-24

Raghavendra AS, Padmasree K (2003) Beneficial interactions of mitochondrial metabolism with photosynthetic carbon assimilation. Trends Plant Sci 8:546-553

Ralph PJ, Burchett MD (1998) Photosynthetic response of Halophila ovalis to heavy metal stress. Environ Pollut 103:91-101

Ralph PJ, Gademann R (2005) Rapid light curves: a powerful tool to assess photosynthetic activity. Aquat Bot 82:222-237

Ralph PJ, Durako MJ, Enríquez S, Collier CJ, Doblin MA (2007) Impact of light limitation on seagrasses. J Exp Mar Biol Ecol 350:176-193
Reale L, Ferranti F, Mantilacci S, Corboli M, Aversa S, Landucci F, Baldisserotto C, Ferroni L, Pancaldi S, Venanzini R (2016) Cyto-histological and morpho-physiological responses of common duckweed (Lemna minor L.) to chromium. Chemosphere 145:98-105

Renger T, Schlodder E (2011) Optical properties, excitation energy and primary charge transfer in photosystem II: theory meets experiment. J Photochem Photobiol B 104:126-141

Reppert M, Acharya K, Neupane B, Jankowiak R (2010) Lowest electronic states of the CP47 antenna protein complex of photosystem II: simulation of optical spectra and revised structural assignments. J Phys Chem B 114:11884-11898

Rintamäki E, Martinsuo P, Pursiheimo S, Aro E-M (2000) Cooperative regulation of light-harvesting complex II phosphorylation via the plastoquinol and ferredoxin-thioredoxin system in chloroplasts. Proc Natl Acad Sci USA 97:11644-11649

Robakowski P (2005) Susceptibility to low-temperature photoinhibition in three conifers differing in successional status. Tree Physiol 25:1151-1160

Robinson HH, Crofts AR (1983) Kinetics of the oxidation-reduction reactions of photosystem II quinone acceptor complex, and the pathway for deactivation. FEBS Lett 153:221-226

Roháček K (2002) Chlorophyll fluorescence parameters: the definitions, photosynthetic meaning, and mutual relationships. Photosynthetica 40:13-29

Roháček K, Barták M (1999) Technique of the modulated chlorophyll fluorescence: basic concepts, useful parameters, and some applications. Photosynthetica 37:339-363

Roháček K, Soukupová J, Barták M (2008) Chlorophyll fluorescence: a wonderful tool to study plant physiology and plant stress. Res Signpost 37(661):41-104

Romanowska-Duda B, Kalaji MH, Strasser RJ (2005) The use of PSII activity of Spirodela oligorrhiza plants as an indicator for water toxicity. In: Van der Est A, Bruce D (eds) Photosynthesis: fundamental aspects to global perspectives. Allen Press, Lawrence, KS, pp 585-587

Romanowska-Duda ZB, Grzesik M, Kalaji MH (2010) Physiological activity of energy plants fertilized with sewage sludge and usefulness of the Phytotoxkit test in practice. Environ Prot Eng 36:73-81

Rosenqvist E, van Kooten O (2003) Chlorophyll fluorescence: a general description and nomenclature. In: DeEll JR, Toivonen PMA (eds) Practical applications of chlorophyll fluorescence in plant biology. Kluwer Academic Publishers, Dordrecht, pp 31-77

Rosyara UR, Subedi S, Sharma RC, Duveiller E (2010) The effect of spot blotch and heat stress in variation of canopy temperature depression, chlorophyll fluorescence and chlorophyll content of hexaploid wheat genotypes. Euphytica 174:377-390

Rousseau C, Belin E, Bove E, Rousseau D, Fabre F, Berruyer R, Guillaumès J, Manceau C, Jacques M-A, Boureau T (2013) High throughput quantitative phenotyping of plant resistance using chlorophyll fluorescence image analysis. Plant Methods 9:17. doi:10.1186/1746-4811-9-17

Ruban AV, Horton P (1992) Mechanism of delta-pH-dependent dissipation of absorbed excitation-energy by photosynthetic membranes. 1. Spectroscopic analysis of isolated light-harvesting complexes. Biochim Biophys Acta 1102:30-38

Rutherford AW, Govindjee, Inoue Y (1984) Charge accumulation and photochemistry in leaves studied by thermoluminescence and delayed light emission. Proc Natl Acad Sci USA 81:1107-1111

Sacksteder CA, Kanazawa A, Jacoby ME, Kramer DM (2000) The proton to electron stoichiometry of steady-state photosynthesis in living plants: in proton-pumping $\mathrm{Q}$ cycle is continuously engaged. Proc Natl Acad Sci USA 97:14283-14288

Salvucci ME, Crafts-Brandner SJ (2004) Inhibition of photosynthesis by heat stress: the activation state of Rubisco as a limiting factor in photosynthesis. Physiol Plant 120:179-186 
Samborska IA, Alexandrov V, Sieczko L, Kornatowska B, Goltsev V, Cetner MD, Kalaji MH (2014) Artificial neural networks and their application in biological and agricultural research. Signpost Open Access J NanoPhotoBioSciences 2:14-30

Samson G, Bruce D (1996) Origins of the low yield of chlorophyll $a$ fluorescence induced by a single turnover flash in spinach thylakoids. Biochim Biophys Acta 1276:147-153

Sarvikas P, Tyystjärvi T, Tyystjärvi E (2010) Kinetics of prolonged photoinhibition revisited: photoinhibited photosystem II centres do not protect the active ones against loss of oxygen evolution. Photosynth Res 103:7-17

Satoh K (1981) Fluorescence induction and activity of ferredoxin$\mathrm{NADP}^{+}$reductase in Bryopsis chloroplasts. Biochim Biophys Acta 638:327-333

Saura P, Quiles MJ (2009) Assessment of photosynthesis tolerance to herbicides, heat and high illumination by fluorescence. Open Plant Sci J 3:7-13

Savikhin S (2006) Ultrafast optical spectroscopy of photosystem I. In: Golbeck JH (ed) Photosystem I: the light-driven plastocyanin: ferredoxin oxidoreductase. Springer, Dordrecht, pp 155-175

Sazanov LA, Burrows PA, Nixon PJ (1998) The chloroplast Ndh complex mediates the dark reduction of the plastoquinone pool in response to heat stress in tobacco leaves. FEBS Lett 429:115-118

Schaller S, Latowski D, Jemioła-Rzemińska M, Dawood A, Wilhelm C, Strzałka K, Goss R (2011) Regulation of LHCII aggregation by different thylakoid membrane lipids. Biochim Biophys Acta 1807:326-335

Schansker G, Strasser RJ (2005) Quantification of non-Q $\mathrm{Q}_{\mathrm{B}}$-reducing centers in leaves using a far-red pre-illumination. Photosynth Res 84:145-151

Schansker G, Goussias C, Petrouleas V, Rutherford AW (2002) Reduction of the Mn cluster of the water-oxidizing enzyme by nitric oxide: formation of an S-2 state. Biochem US 41:3057-3064

Schansker G, Srivastava A, Strasser RJ (2003) Characterization of the 820-nm transmission signal paralleling the chlorophyll $a$ fluorescence rise (OJIP) in pea leaves. Funct Plant Biol 30:785-796

Schansker G, Tóth SZ, Strasser RJ (2005) Methylviologen and dibromothymoquinone treatments of pea leaves reveal the role of photosystem I in the Chl $a$ fluorescence rise OJIP. Biochim Biophys Acta 1706:250-261

Schansker G, Tóth SZ, Strasser RJ (2006) Dark-recovery of the Chl $a$ fluorescence transient (OJIP) after light adaptation: the qTcomponent of non-photochemical quenching is related to an activated photosystem I acceptor side. Biochim Biophys Acta 1757:787-797

Schansker G, Yuan Y, Strasser RJ (2008) Chl $a$ fluorescence and $820 \mathrm{~nm}$ transmission changes occurring during a dark-to-light transition in pine needles and pea leaves: a comparison. In: Allen JF, Osmond B, Golbeck JH, Gantt E (eds) Photosynthesis. Energy from the sun. Springer, Dordrecht, pp 951-955

Schansker G, Tóth SZ, Kovács L, Holzwarth AR, Garab G (2011) Evidence for a fluorescence yield change driven by a lightinduced conformational change within photosystem II during the fast chlorophyll $a$ fluorescence rise. Biochim Biophys Acta 1807:1032-1043

Schansker G, Tóth SZ, Holzwarth AR, Garab G (2014) Chlorophyll $a$ fluorescence: beyond the limits of the $\mathrm{Q}_{\mathrm{A}}$-model. Photosynth Res 120:43-58

Scharte J, Schön H, Weis E (2005) Photosynthesis and carbohydrate metabolism in tobacco leaves during an incompatible interaction with Phytophthora nicotianae. Plant Cell Environ 28:1421-1435

Schatz GH, Brock H, Holzwarth AR (1988) Kinetic and energetic model for the primary processes in photosystem II. Biophys J 54:397-405
Scheibe R, Backhausen JE, Emmerlich V, Holtgrefe S (2005) Strategies to maintain redox homeostasis during photosynthesis under changing conditions. J Exp Bot 56:1481-1489

Schreiber U (1986) Detection of rapid induction kinetics with a new type of high-frequency modulated chlorophyll fluorometer. Photosynth Res 9:261-272

Schreiber U, Berry JA (1977) Heat-induced changes of chlorophyll fluorescence in intact leaves correlated with damage of the photosynthetic apparatus. Planta 136:233-238

Schreiber U, Bilger W (1987) Rapid assessment of stress effects on plant leaves by chlorophyll fluorescence measurements. In: Tenhunen JD, Catarino FM, Lange OL, Oechel WC (eds) Plant response to stress, NATO ASI series, vol 15. Springer, Berlin, pp 27-53

Schreiber U, Krieger A (1996) Two fundamentally different types of variable chlorophyll fluorescence in vivo. FEBS Lett 397:131-135

Schreiber U, Neubauer C (1987) The polyphasic rise of chlorophyll fluorescence upon onset of strong continuous illumination: II. Partial control by the photosystem II donor side and possible ways of interpretation. Z Naturforsch 42c:1255-1264

Schreiber U, Schliwa U, Bilger W (1986) Continuous recording of photochemical and non-photochemical chlorophyll fluorescence quenching with a new type of modulation fluorometer. Photosynth Res 10:51-62

Schreiber U, Endo T, Mi H, Asada K (1995) Quenching analysis of chlorophyll fluorescence by the saturation pulse method: particular aspects relating to the study of eukaryotic algae and cyanobacteria. Plant Cell Physiol 36:873-882

Schreiber U, Klughammer C, Kolbowski J (2012) Assessment of wavelength-dependent parameters of photosynthetic electron transport with a new type of multi-color PAM chlorophyll fluorometer. Photosynth Res 113:127-144

Schultz HR (1996) Leaf absorptance of visible radiation in Vitis vinifera $\mathrm{L}$.: estimates of age and shade effects with a simple field method. Sci Hortic 66:93-102

Schulze ED (1986) Carbon dioxide and water vapor exchange in response to drought in the atmosphere and in the soil. Annu Rev Plant Physiol 37:247-274

Sharkey TD, Zhang R (2010) High temperature effects on electron and proton circuits of photosynthesis. J Integr Plant Biol 52:712-722

Shikanai T (2014) Central role of cyclic electron transport around photosystem I in the regulation of photosynthesis. Curr Opin Biotechnol 26:25-30

Shinkarev VP (2005) Flash-induced oxygen evolution in photosynthesis: simple solution for the extended S-state model that includes misses, double-hits, inactivation, and backward-transitions. Biophys J 88:412-421

Shubin VV, Bezsmertnaya IN, Karapetyan NV (1992) Isolation from Spirulina membranes of two photosystem I-type complexes, one of which contains chlorophyll responsible for the $77 \mathrm{~K}$ fluorescence band at $760 \mathrm{~nm}$. FEBS Lett 309:340-342

Shubin VV, Terekhova IN, Kirillova BA, Karapetyan NV (2008) Quantum yield of $\mathrm{P} 700^{+}$photodestruction in isolated photosystem I complexes of the cyanobacterium Arthrospira platensis. Photochem Photobiol Sci 7:956-962

Šiffel P, Braunová Z (1999) Release and aggregation of the light harvesting complex in intact leaves subjected to strong $\mathrm{CO}_{2}$ deficit. Photosynth Res 61:217-226

Šimić D, Lepeduš H, Jurković V, Antunović J, Cesar V (2014) Quantitative genetic analysis of chlorophyll $a$ fluorescence parameters in maize in the field environments. J Integr Plant Biol 56:695-708

Sinclair J, Spence SM (1990) Heterogeneous photosystem 2 activity in isolated spinach chloroplasts. Photosynth Res 24:209-220

Skotnica J, Fiala J, Ilík P, Dvořk L (1999) Thermally induced chemiluminescence of barley leaves. Photochem Photobiol 69:211-217 
Smit MF, van Heerden PDR, Pienaar JJ, Weissflog L, Strasser RJ, Krüger GHJ (2009) Effect of trifluoroacetate, a persistent degradation product of fluorinated hydrocarbons, on Phaseolus vulgaris and Zea mays. Plant Physiol Biochem 47:623-634

Snel JFH, Dassen HHA (2000) Measurement of light and $\mathrm{pH}$ dependence of single-cell photosynthesis by fluorescence microscopy. J Fluoresc 10:269-273

Snel JFH, Kooijman M, Vredenberg WJ (1990) Correlation between chlorophyll fluorescence and photoacoustic signal transients in spinach leaves. Photosynth Res 25:259-268

Søbye KW, Streibig JC, Cedergreen N (2011) Prediction of joint herbicide action by biomass and chlorophyll $a$ fluorescence. Weed Res 51:23-32

Solymosi K (2012) Plastid structure, diversification and interconversions. I. Algae. Curr Chem Biol 6:167-186

Sonneveld A, Rademaker H, Duysens LNM (1980) Microsecond delayed fluorescence of photosystem II of photosynthesis in various algae: emission spectra and uphill energy transfer. FEBS Lett 113:323-327

Srivastava A, Greppin H, Strasser RJ (1995) Acclimation of land plants to diurnal changes in temperature and light. In: Mathis $\mathrm{P}$ (ed) Photosynthesis: from light to biosphere, vol IV. Kluwer, Dordrecht, pp 909-912

Srivastava A, Guissé B, Greppin H, Strasser RJ (1997) Regulation of antenna structure and electron transport in Photosystem II of Pisum sativum under elevated temperature probed by the fast polyphasic chlorophyll $a$ fluorescence transient: OKJIP. Biochim Biophys Acta 1320:95-106

Srivastava A, Strasser RJ, Govindjee (1999) Greening of peas: parallel measurements of $77 \mathrm{~K}$ emission spectra, OJIP chlorophyll $a$ fluorescence transient, period four oscillation of the initial fluorescence level, delayed light emission, and P700. Photosynthetica 37:365-392

StatSoft, Inc. (2011). STATISTICA (data analysis software system), version 10. www.statsoft.com

Steffen R, Christen G, Renger G (2001) Time-resolved monitoring of flash-induced changes of fluorescence quantum yield and decay of delayed light emission in oxygen-evolving photosynthetic organisms. Biochemistry 40:163-180

Steffen R, Eckert H-J, Kelly AA, Dörmann P, Renger G (2005) Investigations on the reaction pattern of photosystem II in leaves from Arabidopsis thaliana by time-resolved fluorometric analysis. Biochemistry 44:3123-3133

Stewart DW, Costa C, Dwyer LM, Smith DL, Hamilton RI, Ma BL (2003) Canopy structure, light interception, and photosynthesis in maize. Agronom J 95:1465-1474

Stirbet A (2013) Excitonic connectivity between photosystem II units: what is it, and how to measure it? Photosynth Res 116:189-214

Stirbet A, Govindjee (2012) Chlorophyll $a$ fluorescence induction: a personal perspective of the thermal phase, the J-I-P rise. Photosynth Res 113:15-61

Stirbet A, Govindjee (2016) The slow phase of chlorophyll $a$ fluorescence induction in silico: origin of the S-M fluorescence rise. Photosynth Res 130:193-213

Strand M, Lundmark T (1987) Effects of low night temperature and light on chlorophyll fluorescence of field-grown seedlings of Scots pine (Pinus sylvestris L.). Tree Physiol 3:211-224

Strasser RJ (1978) The grouping model of plant photosynthesis. In: Akoyunoglou G, Argyroudi-Akoyunoglou (eds) Chloroplast development. Elsevier/North Holland Biomedical Press, The Netherlands, pp 513-524

Strasser RJ, Butler WL (1977) Fluorescence emission spectra of photosystem I, photosystem II and the light-harvesting chlorophyll $\mathrm{a} / \mathrm{b}$ complex of higher plants. Biochim Biophys Acta 462:307-313

Strasser RJ, Stirbet A (2001) Estimation of the energetic connectivity of PS II centres in plants using the fluorescence rise O-J-I-P: fitting of experimental data to three different PS II models. Math Comput Simul 56:451-462

Strasser BJ, Strasser RJ (1995) Measuring fast fluorescence transients to address environmental questions: The JIP test. In: Mathis P (ed) Photosynthesis: from light to biosphere, vol V. Kluwer Acadamic Publishers, Dordrecht, pp 977-980

Strasser BJ, Strasser RJ (1998) Oscillations of the chlorophyll $a$ fluorescence related to the S-states of the oxygen evolving complex. In: Garab G (ed) Photosynthesis: mechanisms and effects, vol V. Kluwer, Dordrecht, pp 4325-4328

Strasser RJ, Srivastava A, Tsimilli-Michael M (2000) The fluorescence transient as a tool to characterize and screen photosynthetic samples. In: Yunus M, Pathre U, Mohanty P (eds) Probing photosynthesis: mechanisms, regulation and adaptation. Tayor \& Francis, London, pp 445-483

Strasser RJ, Schansker G, Srivastava A, Govindjee (2001) Simultaneous measurement of photosystem I and photosystem II probed by modulated transmission at $820 \mathrm{~nm}$ and by chlorophyll a fluorescence in the sub ms to second time range. In: Proceedings of the 12th international congress on photosynthesis. CSIRO Publishing, Collingwood, Australia, S14-003

Strasser RJ, Tsimilli-Michael M, Srivastava A (2004) Analysis of the chlorophyll $a$ fluorescence transient. In: Papageorgiou G, Govindjee (eds) Chlorophyll $a$ Fluorescence: a signature of photosynthesis, advances in photosynthesis and respiration. Springer, Dordrecht, pp 321-362

Strasser RJ, Tsimilli-Michael M, Qiang S, Goltsev V (2010) Simultaneous in vivo recording of prompt and delayed fluorescence and 820-nm reflection changes during drying and after rehydration of the resurrection plant Haberlea rhodopensis. Biochim Biophys Acta 1797:1313-1326

Strehler BL, Arnold WA (1951) Light production by green plants. J Gen Physiol 34:809-820

Sun R, Liu K, Dong L, Wu Y, Paulsen H, Yang C (2015) Direct energy transfer from the major antenna to the photosystem II core complexes in the absence of minor antennae in liposomes. Biochim Biophys Acta 1847:248-261

Suorsa M, Jarvi S, Grieco M, Nurmi M, Pietrzykowska M, Rantala M, Kangasjarvi S, Paakkarinen V, Tikkanen M, Jansson S, Aro EM (2012) PROTON GRADIENT REGULATION5 is essential for proper acclimation of Arabidopsis photosystem I to naturally and artificially fluctuating light conditions. Plant Cell 24:2934-2948

Suorsa M, Rantala M, Mamedov F, Lespinasse M, Trotta A, Grieco M, Vuorio E, Tikkanen M, Järvi S, Aro EM (2015) Light acclimation involves dynamic re-organisation of the pigmentprotein megacomplexes in non-appressed thylakoid domains. Plant J 84:360-373

Suresh K, Nagamani C, Kantha DL, Kumar MK (2012) Changes in photosynthetic activity in five common hybrids of oil palm (Elaeis guineensis Jacq.) seedlings under water deficit. Photosynthetica 50:549-556

Swoczyna T, Kalaji MH, Pietkiewicz S, Borowski J, ZaraśJanuszkiewicz E (2010a) Photosynthetic apparatus efficiency of eight tree taxa as an indicator of their tolerance to urban environments. Dendrobiology 63:65-75

Swoczyna T, Kalaji MH, Pietkiewicz S, Borowski J, ZaraśJanuszkiewicz E (2010b) Monitoring young urban trees tolerance to roadside conditions by application of chlorophyll fluorescence technique. Zesz Probl Post N Rol 545:303-309

Tallman G (2004) Are diurnal patterns of stomatal movement the result of alternating metabolism of endogenous guard cell ABA and accumulation of ABA delivered to the apoplast around guard cells by transpiration? J Exp Bot 55:1963-1976

Tikkanen M, Aro EM (2014) Integrative regulatory network of plant thylakoid energy transduction. Trends Plant Sci 19:10-17 
Tikkanen M, Mekala NR, Aro E-M (2014) Photosystem II photoinhibition-repair cycle protects photosystem I from irreversible damage. Biochim Biophys Acta 1837:210-215

Tikkanen M, Rantala S, Aro E-M (2015) Electron flow from PSII to PSI under high light is controlled by PGR5 but not by PSBS. Front Plant Sci 6:521. doi:10.3389/fpls.2015.00521

Tiwari A, Mamedov F, Grieco M, Suorsa M, Jajoo A, Styring S, Tikkanen M, Aro E-M (2016) Photodamage of iron-sulphur clusters in photosystem I induces non-photochemical energy dissipation. Nat Plants. doi:10.1038/NPLANTS.2016.35

Tóth SZ, Strasser RJ (2005) The specific rate of $\mathrm{Q}_{\mathrm{A}}$ reduction increases as a function of the initial $\mathrm{Q}_{\mathrm{A}}^{-}$concentration. In: van Est A, Bruce D (eds) Photosynthesis: fundamental aspects to global perspectives. Allen Press, Lawrence, pp 198-200

Tóth SZ, Schansker G, Kissimon J, Kovács L, Garab G, Strasser RJ (2005a) Biophysical studies of photosystem II-related recovery processes after a heat pulse in barley seedling (Hordeum vulgare L.). J Plant Physiol 162:181-194

Tóth SZ, Schansker G, Strasser RJ (2005b) In intact leaves, the maximum fluorescence level $\left(F_{M}\right)$ is independent of the redox state of the plastoquinone pool: a DCMU-inhibition study. Biochim Biophys Acta 1708:275-282

Tóth SZ, Schansker G, Garab G, Strasser RJ (2007a) Photosynthetic electron transport activity in heat-treated barley leaves: the role of internal alternative electron donors to photosystem II. Biochim Biophys Acta 1767:295-305

Tóth SZ, Schansker G, Strasser RJ (2007b) A non-invasive assay of the plastoquinone pool redox state based on the OJIP-transient. Photosynth Res 93:193-203

Tóth SZ, Puthur JT, Nagy V, Garab G (2009) Experimental evidence for ascorbate-dependent electron transport in leaves with inactive oxygen-evolving complexes. Plant Physiol 149:1568-1578

Tóth SZ, Schansker G, Garab G (2013) The physiological roles and metabolism of ascorbate in chloroplasts. Physiol Plant 148:161-175

Trissl H-W, Lavergne J (1995) Fluorescence induction from photosystem II: analytical equations for the yields of photochemistry and fluorescence derived from analysis of a model including exciton-radical pair equilibrium and restricted energy transfer between photosynthetic units. Aust J Plant Physiol 22:183-193

Trissl HW, Wilhelm C (1993) Why do thylakoid membranes from higher plants form grana stacks? Trends Biochem Sci 18:415-419

Tuba Z, Saxena DK, Srivastava K, Singh S, Sz Czebol, Kalaji MH (2010) Chlorophyll $a$ fluorescence measurements for validating the tolerant bryophytes for heavy metal $(\mathrm{Pb})$ biomapping. Curr Sci 98:1505-1508

Tyystjärvi E, Aro EM (1996) The rate constant of photoinhibition, measured in lincomycin-treated leaves, is directly proportional to light intensity. Proc Natl Acad Sci USA 93:2213-2218

Tyystjärvi E, Vass I (2004) Light emission as a probe of charge separation and recombination in the photosynthetic apparatus: relation of prompt fluorescence to delayed light emission and thermoluminescence. In: Papageorgiou GC, Govindjee (eds) Chlorophyll $a$ fluorescence: a signature of photosynthesis. Advances in photosynthesis and respiration, vol 19. Springer, Dordrecht, pp 363-388

Tyystjärvi E, Koski A, Keränen M, Nevalainen O (1999) The Kautsky curve is a built-in barcode. Biophys J 77:1159-1167

van der Weij-de Wit CD, Ihalainen JA, van Grondelle R, Dekker JP (2007) Excitation energy transfer in native and unstacked thylakoid membranes studied by low temperature and ultrafast fluorescence spectroscopy. Photosynth Res 93:173-182

van Dorssen RJ, Breton J, Plijter JJ, Satoh K, van Gorkom HJ, Amesz J (1987) Spectroscopic properties of the reaction center and of the $47 \mathrm{kDa}$ chlorophyll protein of photosystem II. Biochim Biophys Acta 893:267-274 van Grondelle R, Gobets B (2004) Transfer and trapping of excitations om plant photosystems. In: Papageorgiou GC, Govindjee (eds) Chlorophyll $a$ fluorescence: a signature of photosynthesis. Advances in photosynthesis and respiration, vol 19. Springer, Dordrecht, pp 107-132

van Kooten O, Snel JFH (1990) The use of chlorophyll fluorescence nomenclature in plant stress physiology. Photosynth Res $25: 147-150$

van Rensen JJS, Vredenberg WJ, Rodrigues GC (2007) Time sequence of the damage to the acceptor and donor sides of photosystem II by UV-B radiation as evaluated by chlorophyll $a$ fluorescence. Photosynth Res 94:291-297

Vass I (2003) The history of photosynthetic thermoluminescence. Photosynth Res 76:303-318

Vass I, Govindjee (1996) Thermoluminescence from the photosynthetic apparatus. Photosynth Res 48:117-126

Vass I, Horváth G, Herczeg T, Demeter S (1981) Photosynthetic energy conservation investigated by thermoluminescence; activation energies and half-lives of thermoluminescence bands of chloroplasts determined by mathematical resolution of glow curves. Biochim Biophys Acta 634:140-152

Vass I, Kirilovski D, Etienne A-L (1999) UV-B radiation-induced donor- and acceptor-side modifications of photosystem II in the cyanobacterium Synechocystis sp. PCC 6803. Biochemistry 38:12786-12794

Velthuys BR, Visser JWM (1975) The reactivation of EPR signal II in chloroplasts treated with reduced dichlorophenol-indophenol: evidence against a dark equilibrium between two oxidation states of the oxygen evolving system. FEBS Lett 55:109-112

Vermaas WFJ, Renger G, Dohnt G (1984) The reduction of the oxygen-evolving system in chloroplasts by thylakoid components. Biochim Biophys Acta 764:194-202

Veselovskii V, Veselova T (1990) Plant Luminescence: theoretical and practical aspects. Nauka, Moscow [in Russian]

von Caemmerer S (2000) Biochemical models of photosynthesis. Techniques in plant sciences, vol 2. CSIRO Publications, Collingwood

Vos MH, van Gorkom HJ, van Leeuwen PJ (1991) An electroluminescence study of stabilization reactions in the oxygen-evolving complex of photosystem II. Biochim Biophys Acta 1056:27-39

Vredenberg WJ (2008) Analysis of initial chlorophyll fluorescence induction kinetics in chloroplasts in terms of rate constants of donor side quenching release and electron trapping in photosystem II. Photosynth Res 96:83-97

Wagner R, Dietzel L, Bräutigam K, Fischer W, Pfannschmidt T (2008) The long-term response to fluctuating light quality is an important and distinct light acclimation mechanism that supports survival of Arabidopsis thaliana under low light conditions. Planta 228:573-587

Warburg O, Negelein E (1923) Über den Einfluss der Wellenlänge auf den Energieumsatz bei der Kohlensäureassimilation. Z Physik Chem 106:191-218 [in German]

Weis E, Berry JA (1987) Quantum efficiency of photosystem II in relation to 'energy'-dependent quenching of chlorophyll fluorescence. Biochim Biophys Acta 894:198-208

White AJ, Critchley C (1999) Rapid Light Curves: a new fluorescence method to assess the state of the photosynthetic apparatus. Photosynth Res 59:63-72

Wientjes E, van Amerongen H, Croce R (2013a) Quantum yield of charge separation in photosystem II: functional effect of changes in the antenna size upon light acclimation. J Phys Chem B 117:11200-11208

Wientjes E, van Amerongen H, Croce R (2013b) LHCII is an antenna of both photosystems after long-term acclimation. Biochim Biophys Acta 1827:420-426 
Wilson KG, Ralph PJ (2012) Laboratory testing protocol for the impact of dispersed petrochemicals on seagrass. Mar Pollut Bull 64:2421-2427

Wingler A, Brownhill E, Portau N (2005) Mechanisms of the lightdependent induction of cell death in tobacco plants with delayed senescence. J Exp Bot 56:2897-2905

Witt HT (1979) Energy conversion in the functional membranes of photosynthesis analysis by light pulse and electric pulse methods. Biochim Biophys Acta 505:355-427

Wraight CA, Crofts AR (1971) Delayed fluorescence and the highenergy state of chloroplasts. Eur J Biochem 19:386-397

Yamane Y, Kashino Y, Koike H, Satoh K (1998) Effects of high temperatures on the photosynthetic systems in spinach: oxygenevolving activities, fluorescence characteristics and the denaturation process. Photosynth Res 57:51-59

Yamauchi Y, Sugimoto Y (2010) Effect of protein modification by malondialdehyde on the interaction between the oxygen-evolving complex $33 \mathrm{kDa}$ protein and photosystem II core proteins. Planta 231:1077-1088

Yang DL, Jing RL, Chang XP, Li W (2007) Quantitative trait loci mapping for chlorophyll fluorescence and associated traits in wheat (Triticum aestivum). OMICS 49:646-654

Yanniccari M, Tambussi E, Istilart C, Castro AM (2012) Glyphosate effects on gas exchange and chlorophyll fluorescence responses of two Lolium perenne L. biotypes with differential herbicide sensitivity. Plant Physiol Biochem 57:210-217

Yin Z, Meng F, Song H, He X, Xu X, Yu D (2010) Mapping quantitative trait loci associated with chlorophyll $a$ fluorescence parameters in soybean (Glycine $\max$ (L.) Merr.). Planta 231:875-885

Yocum CF (2008) The calcium and chloride requirements of the $\mathrm{O}_{2}$ evolving complex. Coord Chem Rev 252:296-305

Yokono M, Takabayashi A, Akimoto S, Tanaka A (2015) A megacomplex composed of both photosystem reaction centres in higher plants. Nat Commun 6:6675. doi:10.1038/ncomms7675
Yoshida K, Terashima I, Noguchi K (2007) Up-regulation of mitochondrial alternative oxidase concomitant with chloroplast over-reduction by excess light. Plant Cell Physiol 48:606-614

Yusuf MA, Kumar D, Rajwanshi R, Strasser RJ, Tsimilli-Michael M, Govindjee, Sarin NM (2010) Overexpression of y-tocopherol methyl transferase gene in transgenic Brassica juncea plants alleviates abiotic stress: physiological and chlorophyll fluorescence measurements. Biochim Biophys Acta 1791:428-438

Zaharieva I, Goltsev V (2003) Advances on photosystem II investigation by measurement of delayed chlorophyll fluorescence by a phosphoroscopic method. Photochem Photobiol 77:292-298

Zhang ZB, Xu P, Jia JZ, Zhou RH (2010) Quantitative trait loci for leaf chlorophyll fluorescence traits in wheat. Aust J Crop Sci 4:571-579

Živčák M, Brestič M, Olšovská K (2008a) Physiological parameters useful in screening for improved tolerance to drought in winter wheat (Triticum aestivum L.). Cereal Res Commun 36:1943-1946

Živčák M, Brestič M, Olšovská K (2008b) Application of photosynthetic parameters in screening of wheat (Triticum aestivum L.) genotypes for improved drought and high temperature tolerance. In: Allen JF, Gantt E, Goldbeck JH, Osmond B (eds) Photosynthesis. Energy from the sun. Springer, Dordrecht, pp 1247-1250

Živčák M, Brestič M, Olšovská K, Slamka P (2008c) Performance Index as a sensitive indicator of water stress in Triticum aestivum. Plant Soil Environ 54:133-139

Živčák M, Brestič M, Balatová Z, Drevenaková P, Olsovska K, Kalaji HM, Allakhverdiev SI (2013) Photosynthetic electron transport and specific photoprotective responses in wheat leaves under drought stress. Photosynth Res 117:529-546

Živčák M, Kalaji HM, Shao HB, Olšovská K, Brestič M (2014) Photosynthetic proton and electron transport in wheat leaves under prolonged moderate drought stress. J Photochem Photobiol B 137:107-115 